\title{
Nonlinear Modeling and Simulation of Tumor Growth
}

Vittorio Cristini ${ }^{1, \star}$, Hermann B. Frieboes ${ }^{1,2}$, Xiaongrong $\mathrm{Li}^{3}$, John S. Lowengrub $^{2,3, \star \star}$, Paul Macklin ${ }^{1}$, Sandeep Sanga ${ }^{1,4}$, Steven M. Wise ${ }^{2}$, and Xiaoming Zheng ${ }^{2}$

1 School of Health Information Sciences, The University of Texas Health Science Center at Houston,

2 Department of Mathematics, University of California, Irvine,

3 Department of Biomedical Engineering, University of California, Irvine,

4 Department of Biomedical Engineering, University of Texas, Austin

\section{Introduction}

The effects of the interaction between cellular- and tumor-scale processes on cancer progression and treatment response remain poorly understood (for instance, the crucial role of the microenvironment in cancer growth and invasion $[95,65,184,183,160,110,66,166])$. Three-dimensional tissue morphology, cell phenotype, and molecular phenomena are intricately coupled; they influence cancer invasion potential by controlling tumor-cell proliferation and migration [78, 187, 198]. Hypoxia [88, 210, 186, 70, 91], acidosis [91, 199, 96], and associated diffusion gradients, caused by heterogeneous delivery of oxygen and nutrients and removal of metabolites [104, 103] due to highly disorganized microvasculature [92, 106] and often exacerbated by therapy (e.g., anti-angiogenic $[160,177])$, can induce heterogeneous spatial distribution and invasiveness of tumor cells through a variety of molecular $[175,209,208,44,165,173,145$, 118, 28, 29, 190, 156, 185, 120, 122, 24, 160, 177, 174, 61] and tissue-scale $[59,127,72]$ mechanisms corresponding to different tissue-scale invasive patterns $[78,164,194,167,111,187,198,204,117,206,64,179,75,77,53,172]$. Such complex systems, dominated by large numbers of processes and highly nonlinear dynamics, are difficult to approach by experimental methods alone and can typically be better understood only by using appropriate mathematical models and sophisticated computer simulations, complementary to laboratory and clinical observations. Mathematical modeling has the potential

\footnotetext{
* Corresponding Author: V. Cristini - School of Health Information Sciences, University of Texas Health Science Center at Houston, TX 77030, USA. Email: vittorio.cristini@uth.tmc.edu

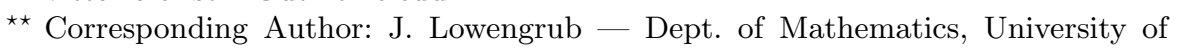
California at Irvine, Irvine, CA 92697. Email: lowengrb@math.uci.edu
} 
to provide insight into these interactions through studies based on physical processes that treat cancer as a system. Our basic hypothesis, supported by an integrative approach combining sophisticated mathematical models with in vitro and in vivo experiments, is that the characteristics of the tumorhost interface can be used to predict the underlying dynamical interactions between tumor cell proliferation and adhesion, which, in turn, reflect both micro-environmental factors and cellular gene expression.

Over the past 10 years, activity in mathematical modeling and computational (in silico) simulation of cancer has increased dramatically (e.g., reviews such as $[3,26,16,37,181,168])$. A variety of modeling strategies have been developed, each focusing on one or more aspects of cancer. Cellular automata and agent-based modeling, where individual cells are simulated and updated based upon a set of biophysical rules, have been developed to study genetic instability, natural selection, carcinogenesis, and interactions of individual cells with each other and the microenvironment. Because these methods are based on a series of rules for each cell, it is simple to translate biological processes (e.g., mutation pathways) into rules for the model. However, these models can be difficult to study analytically, and computational costs can increase rapidly with the number of cells. Because a 1-mm tumor spheroid may have several hundred thousand cells, these methods could become unwieldy when studying tumors of any significant size. See [10, 4, 138] for examples of cellular automata modeling, and $[139,1]$ for examples of agent-based modeling. In larger-scale systems where the cancer cell population is on the order of 1,000,000 or more, continuum methods may provide a more suitable modeling technique. Early work including [90, 39, 40] used ordinary differential equations to model cancer as a homogeneous population, as well as partial differential equation models restricted to spherical geometries. Linear and weakly nonlinear analyses have been performed to assess the stability of spherical tumors to asymmetric perturbations $[49,42,59,127,16,37]$ in order to characterize the degree of aggression. Various interactions of a tumor with the microenvironment, such as stress-induced limitations to growth, have also been studied $[108,6,7,176,17,18,5]$. Most of the modeling has considered single-phase (e.g., single cell species) tumors, although multiphase mixture models have also been developed to provide a more detailed account of tumor heterogeneity [7, 43, 50].

Recently, nonlinear modeling has been performed to study the effects of morphology instabilities on both avascular and vascular solid tumor growth. Results from this research will be the focus of this chapter, subdivided into four sections. Cristini et al. (2003) [59] used boundary integral methods (Sect. 1) to perform the first fully nonlinear simulations of a continuum model of tumor growth in the avascular and vascularized growth stages with arbitrary boundaries. These investigations of the nonlinear regime of shape instabilities predicted encapsulation of external, non-cancerous tissue by morphologically unstable tumors. Li et al. (2007) [127] extended upon [59] in 3-D via an adaptive boundary integral method. Zheng et al. (2005) [212] (Sect. 2) built upon 
the model in [59] to include angiogenesis and an extratumoral microenvironment by developing and coupling a level set implementation with a hybrid continuum-discrete angiogenesis model originally developed by Anderson \& Chaplain (1998) [11]. As in [59], Zheng et al. found that low-nutrient (e.g., hypoxic) conditions could lead to morphological instability. Their work served as a building block for recent studies of the effect of chemotherapy on tumor growth by Sinek et al. (2004) [188] and for studies of morphological instability and invasion by Cristini et al., (2005) [55] and Frieboes et al. (2006) [74]. Hogea et al. (2006) [97] have also begun exploring tumor growth and angiogenesis using a level set method coupled with a continuum model of angiogenesis. Macklin \& Lowengrub (2007) (Sect. 3) used a ghost cell/level set method [136] for evolving interfaces to study tumor growth in heterogeneous tissue and further studied tumor growth as a function of the microenvironment [135]. Finally, Wise et al. (in review) [202] and Frieboes et al. (in review) [73] have developed a diffuse interface implementation of solid tumor growth to study the evolution of multiple tumor cell species, which was employed in Frieboes et al. (in press) [72] to model the 3-D vascularized growth of malignant gliomas (brain tumors) (Sect. 4).

\section{Solid Tumor Growth Using a Boundary-Integral Method}

\subsection{Overview}

In [59] and [127] we studied solid tumor growth in the nonlinear regime using boundary integral simulations in 2-D and 3-D to explore complex morphologies. A new formulation of classical models [90, 142, 40, 41] demonstrated that non-necrotic tumor evolution could be described by a reduced set of two parameters that characterize families of solutions. The parameter $G$ describes the relative rate of mitosis to the relaxation mechanisms (cell mobility and cell-to-cell adhesion). The parameter $A$ describes the balance between apoptosis and mitosis. Both parameters also include the effect of vascularization. The analysis revealed that tumor evolution is qualitatively unaffected by the number of spatial dimensions. The 2-D simulations presented in [59] were the first fully nonlinear simulations of a continuum model of tumor growth, although there had been prior work on cellular automata-based simulations of tumor growth (e.g. see [109] and references therein).

Results reveal that the two new dimensionless parameters uniquely subdivide tumor growth into three regimes associated with increasing degrees

\footnotetext{
${ }^{\ddagger}$ Portions of this section are reprinted from Journal of Mathematical Biology, Cristini et al. Vol. 46, pp. 191-224, Copyright 2003 Springer (with kind permission of Springer Science and Business Media), and with permission from Discrete and Continuous Dynamical Systems - Series B, Li et al., Vol. 7, pp. 581-604, Copyright 2007 American Institute of Mathematical Sciences.
} 
of vascularization: low (diffusion dominated), moderate, and high vascularization. Critical conditions exist for which the tumor evolves to nontrivial dormant states or grows self-similarly (i.e., shape invariant). Away from these critical conditions, evolution may be unstable leading to invasive fingering into the external tissues and to topological transitions such as tumor breakup and reconnection. Interestingly, the shape of highly vascularized tumors always stays compact and invasive fingering does not occur, even while growing unbounded. This is in agreement with experimental observations [151] of in vivo tumor growth and suggests that invasive growth of highly vascularized tumors is associated to vascular and elastic anisotropies (such as substrate inhomogeneities). Existence of non-trivial dormant states was recently proved [81], but no examples of such states were given until this work. The self-similar behavior described here is analogous to that recently found in diffusional crystal growth [58], and leads to the possibility of shape control and of controlling the release of tumor angiogenic factors by restricting the tumor volume-tosurface-area ratio. This could restrict angiogenesis during growth.

In the model, a tumor is treated as an incompressible fluid, and tissue elasticity is neglected. Cell-to-cell adhesive forces are modeled by a surface tension at the tumor-tissue interface. Growth of tumor mass is governed by a balance between cell mitosis and apoptosis (programmed cell-death). The rate of mitosis depends on concentration of nutrient and growth inhibitor factors that obey reaction-diffusion equations in the tumor volume. The bulk source of factors is the blood. The concentration of capillaries in the tumor is assumed to be uniform, as are concentrations of factors in the external tissues. This work focused on the case of nonnecrotic tumors [38] with no inhibitor factors. These conditions apply to small-sized tumors or when nutrient concentrations in the blood and in external tissues are high. Such a model should over-predict growth away from these conditions.

To simulate solid tumor growth in 3-D [127], we developed a new, adaptive boundary integral method by extending to $3-\mathrm{D}$ the continuum model in [59]. The 3-D problem is considerably more difficult owing to the singularities of the integrals and the 3-D surface geometry. In 2-D, it is possible to develop a spectrally accurate numerical algorithm together with a non-stiff time updating scheme for the tumor-host interface position. The numerical method relies on accurate discretizations of singular surface integrals, a spatial rescaling and the use of an adaptive surface mesh originally developed by [54]. In both 2-D and $3-\mathrm{D}$, discretized boundary integral equations are solved iteratively using GMRES, and a discretized version of the Dirichlet-Neumann map is used to determine the normal velocity of the tumor surface. In $3-\mathrm{D}$, a version of the Dirichlet-Neumann map is used that relies on a vector potential formulation rather than a more standard double-layer potential. The vector potential has the advantage that singularity subtraction can be used to increase the order of accuracy of the numerical quadrature. Because of the difficulties in implementing an implicit time-stepping algorithm in 3-D, we instead used explicit 
time stepping with numerical fitting, which allows us to achieve acceptably large time steps.

Although the mathematical tumor model considered here is highly simplified, this work provides a benchmark to assess the effects of additional biophysical processes not considered, such as necrosis, multiple tumor cell types, tissue stress, angiogenesis and a developing neovasculature as well as other microenvironmental features and inhomogeneities. In addition, the boundary integral results serve as a benchmark for validating other numerical methods (e.g. level set, mixture models) that are capable of simulating more complex biophysical processes.

\subsection{Model Formulation}

The model has only one intrinsic length scale, the diffusional length $L_{D}$, and three intrinsic time scales corresponding to the relaxation rate $\lambda_{R}$ (associated to $L_{D}$, cell mobility and cell-to-cell adhesion), the characteristic mitosis rate $\lambda_{M}$ and the apoptosis rate $\lambda_{A}$. The dimensional problem can be reformulated in terms of two nondimensional decoupled problems:

$$
\begin{gathered}
\nabla^{2} \Gamma-\Gamma=0, \quad(\Gamma)_{\Sigma}=1 \\
\nabla^{2} p=0, \quad(p)_{\Sigma}=\kappa-A G \frac{(\mathbf{x} \cdot \mathbf{x})_{\Sigma}}{2 d}
\end{gathered}
$$

in a $d$-dimensional tumor. Time and space have been normalized with the intrinsic scales $L_{D}$ and $\lambda_{R}^{-1}$, the interface $\Sigma$ separates tumor volume from external tissue, and variables $\Gamma$ and $p$ represent a modified nutrient concentration and a modified pressure (see [59] for definitions). Tumor surface $\Sigma$ (of local total curvature $\kappa$ ) is evolved using normal velocity

$$
V=-\mathbf{n} \cdot(\nabla p)_{\Sigma}+G \mathbf{n} \cdot(\nabla \Gamma)_{\Sigma}-A G \frac{\mathbf{n} \cdot(\mathbf{x})_{\Sigma}}{d}
$$

where $\mathbf{n}$ is the outward normal to $\Sigma$ and $\mathbf{x}$ is position in space. The instantaneous problem as stated has only two dimensionless parameters:

$$
G=\frac{\lambda_{M}}{\lambda_{R}}(1-B), \quad A=\frac{\lambda_{A} / \lambda_{M}-B}{1-B}
$$

The former describes the relative strength of cell mitosis to the relaxation mechanisms, and the latter describes the relative strength of cell apoptosis and mitosis. Effects of vascularization are in the parameter $B$ (defined in [59]). Note that in the context of steady solutions, parameter $A$ is related to parameter $\Lambda$ (introduced in [38]) by $A=3 \Lambda$. The rescaled rate of change of tumor volume $H=\int_{\Omega} d x^{d}$ is defined as the mass flux $J=\frac{d}{d t} H=\int_{\Sigma} V d x^{d-1}$. By using (1) and (2) we obtain from (3):

$$
J=-G \int_{\Omega} \Gamma d x^{d}-A G H
$$




\subsection{Regimes of Growth}

Considering evolution of a radially symmetric tumor identifies regimes of growth. The interface $\Sigma$ is an infinite cylinder for $d=2$ or a sphere for $d=3$, with radius $R(t)$. All variables have only $r$-dependence, where $r$ is the polar coordinate. Equations (1)-(2) have the nonsingular solutions

$$
\Gamma(r, t)=\left\{\begin{array}{l}
\frac{I_{0}(r)}{I_{0}(R)}, \quad d=2, \\
\left(\frac{\sinh (R)}{R}\right)^{-1}\left(\frac{\sinh (r)}{r}\right), \quad d=3
\end{array}\right.
$$

and $p(r, t)=(d-1) R^{-1}-A G R^{2} /(2 d)$. Note that $p(r, t) \equiv p(R, t)$, i.e., $p$ is uniform across the tumor volume. From (3) the evolution equation for the tumor radius $R$ is:

$$
\frac{d R}{d t}=V=-A G \frac{R}{d}+G\left\{\begin{array}{l}
\frac{I_{1}(R)}{I_{0}(R)}, \quad d=2, \\
\left(\frac{1}{\tanh (R)}-\frac{1}{R}\right), \quad d=3
\end{array}\right.
$$

For a radially symmetric tumor, $|G|$ rescales time. In all dimensions, unbounded growth $(R \rightarrow \infty)$ occurs if and only if $A G \leq 0$. Growth velocity is plotted for $d=2$ in Fig. 1 . Note that, for $d=3$, results are qualitatively similar and were reported in Fig. 9 in [38], although in the framework of the original formulation the growth regimes had not been identified. The figure is included here to identify the growth regimes. For given $A$, evolution from initial condition $R(0)=R_{0}$ occurs along the corresponding curve. Three regimes are identified, and the behavior is qualitatively unaffected by the number of spatial dimensions $d$.

1. Low vascularization: $G \geq 0$ and $A>0$ (i.e., $\left.B<\lambda_{A} / \lambda_{M}\right)$. Note that the special case of avascular growth $(B=0)$ belongs to this regime. Evolution is monotonic and always leads to a stationary state $R_{\infty}$, that corresponds to the intersection of the curves in Fig. 1 with the dotted line $V=0$. This behavior is in agreement with experimental observations of in vitro diffusional growth [89] of avascular spheroids to a dormant steady state [147, 192]. In the experiments, however, tumors always develop a necrotic core that further stabilizes their growth [39].

2. Moderate vascularization: $G \geq 0$ and $A \leq 0$ (i.e., $1>B \geq \lambda_{A} / \lambda_{M}$ ). Unbounded growth occurs from any initial radius $R_{0}>0$. The growth tends to exponential for $A<0$ with velocity $V \rightarrow-A G R / d$ as $R \rightarrow \infty$, and to linear for $A=0$ with velocity $V \rightarrow G$ as $R \rightarrow \infty$.

3. High vascularization: $G<0$ (i.e., $B>1)$. For $A>0$, growth $(V>0)$ may occur, depending on the initial radius, and is always unbounded; for 
$A<0$ (for which cell apoptosis is dominant: $\lambda_{A} / \lambda_{M}>B$ ), the evolution is always to the only stationary solution $R_{\infty}=0$. This stationary solution may also be achieved for $A>0$. The stationary radius $R_{\infty}$ is independent of $G$, and is a solution of $V=0$ with $V$ from (7). The stationary radius has limiting behaviors

$$
\begin{aligned}
& R_{\infty} \rightarrow d A^{-1}, \quad A \rightarrow 0, \\
& R_{\infty} \rightarrow d^{\frac{1}{2}}(d+2)^{\frac{1}{2}}(1-A)^{\frac{1}{2}}, \quad A \rightarrow 1
\end{aligned}
$$

where $R_{\infty}$ vanishes. Note that the limit $A \rightarrow 1$ corresponds to $\lambda_{A} \rightarrow \lambda_{M}$.

Pressure $P_{C}$ at the center of the tumor $(r \equiv 0)$ can be calculated as (see [59]):

$$
\frac{P_{C}}{\gamma / L_{D}}=(d-1) / R+G-A G R^{2} /(2 d)-G\left\{\begin{array}{l}
1 / I_{0}(R), \quad d=2, \\
R / \sinh (R), \quad d=3
\end{array}\right.
$$

which has asymptotic behavior $P_{C}\left(\gamma / L_{D}\right)^{-1} \rightarrow-A G R^{2} /(2 d)$ as $R \rightarrow \infty$, indicating that if tumors grows unbounded $(A G \leq 0)$ the pressure at the center also does (unless $A=0$ ). This is a direct consequence of the absence of a necrotic core in this model. In reality, increasing pressure may itself contribute to necrosis $[150,163]$. It is known [46] that tumor cells continuously replace the loss of cell volume in a tumor because of necrosis, thus maintaining pressure finite.

\subsection{Linear Analysis}

Consider a perturbation of the spherical tumor interface $\Sigma$ :

$$
R(t)+\delta(t)\left\{\begin{array}{l}
\cos (l \theta), \quad d=2, \\
Y_{l, m}(\theta, \phi), \quad d=3
\end{array}\right.
$$

where $\delta$ is dimensionless perturbation size and $Y_{l, m}$ is a spherical harmonic, where $l$ and $\theta$ are polar wavenumber and angle, and $m$ and $\phi$ are azimuthal wavenumber and angle. By solving the system of (1)-(3) in the presence of a perturbed interface we obtain evolution (7) for unperturbed radius $R$ and perturbation size $\delta$ using the shape factor $\delta / R$ :

$$
\left(\frac{\delta}{R}\right)^{-1} \frac{d \frac{\delta}{R}}{d t}=\left\{\begin{array}{l}
G-\frac{l\left(l^{2}-1\right)}{R^{3}}-G \frac{l+2}{R} \frac{I_{1}(R)}{I_{0}(R)}-G \frac{I_{l+1}(R)}{I_{l}(R)} \frac{I_{1}(R)}{I_{0}(R)}+l \frac{A G}{2}, \quad d=2 \\
G-\frac{l(l+2)(l-1)}{R^{3}} \\
\quad-\left(G \frac{l+3}{R}+G \frac{I_{l+3 / 2}(R)}{l_{l+1 / 2}(R)}\right)\left(\frac{1}{\tanh (R)}-\frac{1}{R}\right)+l \frac{A G}{3}, \quad d=3
\end{array}\right.
$$


Note that linear evolution of the perturbation is independent of azimuthal wavenumber $m$ and there is a critical mode $l_{c}$ such that perturbations grow for $l<l_{c}$ and decay for $l>l_{c}$. The critical mode depends on the parameters $A$, $G$ and the evolving radius $R$. This agrees with the linear analyses presented in $[90,38,41]$ for the special case where the unperturbed configuration is stationary (i.e. $R$ constant).

\subsection{Results}

\section{Unstable Growth in 2-D}

We investigate unstable evolution in the low-vascularization (diffusion dominated) regime, characterized by $G, A>0$, for $d=2$ using nonlinear boundary integral simulations. The linear analysis demonstrates that evolution in the other regimes is stable for $d=2$. In Fig. 2, the evolution of the tumor surface from a nonlinear boundary integral simulation with $N=1024$ and $\triangle t=10^{-3}$ (solid curve) is compared to the result of the linear analysis (dotted). In this case $A=0.5, G=20$, and the initial shape of the tumor is

$$
(x(\alpha), y(\alpha))=(2+0.1 \cos (2 \alpha)(\cos (\alpha), \sin (\alpha))
$$

According to linear theory $((7)$ and Fig. 1), the tumor grows. The radially symmetric equilibrium radius $R_{\infty} \approx 3.32$. Mode $l=2$ is linearly stable initially, and becomes unstable at $R \approx 2.29$. The linear and nonlinear results in Fig. 2 are indistinguishable up to $t=1$, and gradually deviate thereafter. Correspondingly, a shape instability develops and forms a neck. At $t \approx 1.9$ the linear solution collapses suggesting pinch-off; the nonlinear solution is stabilized by the cell-to-cell adhesive forces (surface tension) that resist development of high negative curvatures in the neck. This is not captured by the linear analysis. Instead of pinching off, as is predicted by linear evolution, the nonlinear tumor continues to grow and develops large bulbs that eventually reconnect thus trapping healthy tissue (shaded regions in the last frame in Fig. 2) within the tumor. The frame at $t=2.531$ describes the onset of reconnection of the bulbs. We expect that reconnection would be affected by diffusion of nutrient outside the tumor, which is not included in the model used here. However, predictions of development of shape instabilities and of capture of healthy tissue during growth are in agreement with experimental observations [169].

We have found that during unstable evolution the linear and nonlinear solutions deviate from one another with the nonlinear solution being stabilized against pinch-off and leading instead to tumor reconnection and encapsulation of healthy tissue at later times as observed experimentally [169]. Reconnection occurs even when the amount of apoptosis is increased. 


\section{Unstable Growth in 3-D}

We investigate the nonlinear, unstable evolution of 3-D tumors in the lowvascularization regime characterized by $G \geq 0$ and $A>0$ by focusing on the parameters $G=20$ and $A=0.5$. Note that a spherical tumor with these parameters reaches a steady state with corresponding scale factor $S=4.73$. The evolution is considered using three different initial conditions. In Fig. 3, the morphological evolution of a tumor is shown from the initial radius

$$
r=1+0.033 Y_{2,2}(\theta, \phi)
$$

with the initial scale factor $S(0)=3.002$. Two 3 -D views of the morphology are shown, as indicated. The tumor does not change volume in the simulation because spatial rescaling is used in the algorithm. The associated evolution of the scale factor $S(t)$ is shown in Fig. 4a.

At early times, the perturbation decreases and the tumor becomes spherelike. As the tumor continues to grow, the perturbation starts to increase around time $t \approx 0.4$ when the scale factor $S \approx 3.7$. The tumor then takes on a flattened ellipse like shape. Around time $t \approx 2.2$ when $S \approx 4.6$, the perturbation growth accelerates dramatically, and dimples form around time $t \approx 2.42$. The dimples deepen, and the tumor surface buckles inwards. The instability and dimple formation allow the tumor to increase its surface area relative to its volume, thereby allowing the cells in the tumor bulk greater access to nutrient. This in turn allows the tumor to overcome the diffusional limitations on growth and to grow to larger sizes than would be possible if the tumor were spherical. For example, in Fig. $3 \mathrm{~d}$, the scale factor $S \approx 4.78$, which is larger than the corresponding value (4.73) for the steady state spherical tumor. (Note that the tumor is continuing to grow.) This provides additional support in 3-D for the hypothesis put forth by $[59,55,56]$ based on 2 -D simulations that morphologic instability allows an additional pathway for tumor invasion that does not require an additional nutrient source such as would be provided from a newly developing vasculature through angiogenesis.

In this simulation, the number of mesh points is $N=1024$ initially. As seen in Fig. 3, the mesh adaptively clusters near the dimples where there is large negative curvature thereby providing enhanced local resolution. At the final time $t_{f}=2.67$ (with $S \approx 4.78$ ) there are $N=2439$ nodes on the tumor surface. To compute for longer times, higher resolution is necessary.

We next compare the nonlinear results with the predictions of linear theory. To estimate the shape perturbation size $\bar{\delta}$ in the nonlinear simulation, we take

$$
\bar{\delta}=\max _{\Sigma}\left|r_{\Sigma}-1\right|
$$

since the overall bulk growth is scaled out of the nonlinear evolution by the scale factor $S(t)$ (the maximum is taken over all the computational nodes of the interface).

In Fig. 4b, the results of linear theory (solid) and nonlinear simulations (dashed-dot) for the perturbation size are shown as functions of $S(t)$ for the 
evolution from Fig. 3. Linear theory predicts that for $G=20, A=0.5$, the 2 -mode is stable for $S<3.54$. This is borne out by the nonlinear simulation that agrees very well with the linear theory up until $S \approx 3.5$ where the linear theory predicts the perturbation starts to grow. In the nonlinear simulation, the perturbation continues to decay until $S \approx 3.7$. Although the linear and nonlinear results deviate at larger $S$ with linear theory predicting larger perturbations, the qualitative behavior of the shape perturbation is very similar in both cases. In particular, there is rapid growth of the perturbation near $R \approx 4.6$. The circled points labeled A-D on the dashed-dot curve (nonlinear simulation) correspond to the morphologies shown in Figs. 3a-d. Note that at the final time, the nonlinear perturbation $\bar{\delta} \approx 0.5$, and so the evolution is highly nonlinear.

In Figs. $4 \mathrm{c}$ and $4 \mathrm{~d}$, the nonlinear and linear tumor morphologies, respectively, are shown at time $t=2.668$ where $S \approx 4.78$ and $S_{\text {linear }} \approx 4.73$. The linear morphology is generated by evolving a sphere to the shape prescribed by linear theory. This is why the linear solution is shown with a triangulated mesh. The corresponding nonlinear and linear perturbation sizes are $\bar{\delta}=0.496$ and $\delta / R=0.42$ respectively. Note that the reason the linear result is smaller than the nonlinear value at this time is because the linear scale factor is slightly less than that from the nonlinear simulation which, when combined with the rapid growth of the perturbation around $S \approx 4.76$, gives rise to this behavior.

As seen in Figs. 4c and 4d, the nonlinear tumor morphology is more compact than the corresponding linear result. In fact, the linear perturbation eventually grows so large that the tumor pinches off in the center. In contrast, nonlinearity introduces additional modes that alter the growth directions, from primarily horizontal in Fig. 4d to more vertical in Fig. 4c, thereby avoiding pinch-off and resulting in more compact shapes. This is consistent with the 2-D results of [59].

\section{Nontrivial Stationary States}

We study the case of nontrivial nonlinear stationary states in the lowvascularization regime. That such states exist was recently proved [81] and is predicted by linear theory. To see this, let us consider the linear evolution of a perturbation of a stationary radius $R_{\infty}$. The stationary radius is solution of (7) with $V=0$ (see also the related text) and is a function of $0<A<1$. Thus, the flux $J=0$ and (11) indicates that there exists a critical

$$
G_{l}=\left\{\begin{array}{l}
R_{\infty}^{-3} \frac{2 l\left(l^{2}-1\right)}{2-A\left[2+R_{\infty} I_{l+1}\left(R_{\infty}\right) / I_{l}\left(R_{\infty}\right)\right]}, \quad d=2, \\
R_{\infty}^{-3} \frac{3 l(l-1)(l+2)}{3-A\left[3+R_{\infty} I_{l+3 / 2}\left(R_{\infty}\right) / I_{l+1 / 2}\left(R_{\infty}\right)\right]}, \quad d=3
\end{array}\right.
$$

such that for $G=G_{l}$ the perturbation also remains stationary. It can be shown that for both $d=2$ and $d=3, G_{l}>0$ and a perturbed stationary 
shape always exists. The perturbation $\delta / R_{\infty}$ grows unbounded for $G>G_{l}$ and decays to zero for $G<G_{l}$. At large radii $R_{\infty}, G_{l} \rightarrow 0$; thus, in this limit perturbations of stationary states always grow unbounded.

Next consider the nonlinear evolution for $d=2$ of a mode $l$ perturbation, which is predicted by the linear theory to be stationary. We let the value of $G$ be equal to $G_{l}$, with $R=R_{\infty}$. Although the perturbations are linearly stationary, there is evolution due to nonlinearity. Our results (not shown) strongly suggest that for given $l$ there exists a critical $G_{l}^{\mathrm{NL}}<G_{l}$ such that for $G=G_{l}^{\mathrm{NL}}$ a nonlinear, nontrivial steady shape exists. Thus, nonlinearity is destabilizing for the stationary shapes. This is in contrast with the results obtained earlier where nonlinearity stabilizes the pinch-off predicted by linear theory during unstable evolution. Finally, as expected, the deviation of $G_{l}^{\mathrm{NL}}$ from the linear $G_{l}$ is second order (not shown).

\section{Self-similar evolution}

We investigate conditions for which the tumor grows self-similarly, thus maintaining its shape. This should have implications for angiogenesis, or tumor vascularization. It is known that angiogenesis occurs as tumor angiogenic factors are released, diffusing to nearby vessels and triggering chemotaxis of endothelial cells and thus the formation of a network of blood vessels that finally penetrates the tumor providing nutrients. Assuming the flux of angiogenic factors to be proportional to the tumor/tissue interface area and the rate of production of angiogenic factors to be proportional to the tumor volume, we conclude that self-similar evolution divides tumor growth in two categories: one (stable growth) characterized by a decrease of the area-to-volume ratio during growth thus hampering or preventing angiogenesis, and the other (unstable growth) characterized by an increase of the area-to-volume ratio and thus favoring angiogenesis.

To maintain self-similar evolution during growth we may set $\frac{d}{d t}(\delta / R)=0$ identically. This can be achieved by keeping $G$ constant and varying $A$ as a function of the unperturbed radius $R$ by

$$
A=\left\{\begin{array}{l}
\frac{2\left(l^{2}-1\right)}{G R^{3}}+2\left(l+\frac{2}{l}\right) \frac{1}{R} \frac{I_{1}(R)}{I_{0}(R)}+\frac{2}{l} \frac{I_{l+1}(R) I_{1}(R)}{I_{l}(R) I_{0}(R)}-\frac{2}{l}, \quad d=2, \\
\frac{3(l-1)(l+2)}{G R^{3}}+3\left(l+\frac{3}{l}\right) \frac{1}{R}\left(\frac{1}{\tanh R}-\frac{1}{R}\right) \\
\quad+\frac{3}{l} \frac{I_{l+3 / 2}(R)}{I_{l+1 / 2}(R)}\left(\frac{1}{\tanh R}-\frac{1}{R}\right)-\frac{3}{l}, \quad d=3
\end{array}\right.
$$

In Fig. 5 (top) apoptosis parameter $A(R)$ is shown for $d=2$ (dashed lines) and $d=3$ (solid). Growth velocity corresponding to self similar evolution, obtained from (7) with $A$ given by (16), is plotted in Fig. 5 (bottom). Curves of $A$ divide the plot into regions of stable growth and regions of unstable growth of 
a given mode $l$. Figures 5 top and bottom indicate that in low-vascularization (diffusion-dominated) regime $(G>0, A>0)$ self-similar evolution towards a stationary state is not possible for $G$ constant (stationary states $R_{\infty}$ correspond to intersection of curves (16) in Fig. 5 (top) with the curves describing stationary radii). For instance, growth velocity $V<0$ for initial radius $R_{0}<R_{\infty}$, and thus self-similar shrinkage of the tumor to zero occurs. On the other hand, for $R_{0}>R_{\infty}, V>0$, and thus self-similar growth away from stationary radius occurs. In the high-vascularization regime $(G<0)$, during self-similar evolution the velocity $V<0$. Thus self-similar shrinkage of a tumor from arbitrary initial condition to a point occurs, and self-similar unbounded growth is not possible.

To summarize, self-similar evolution, with the possiblity of shape control, is found in both low and high vascularization regimes with varying $A$ and constant $G$. However, in the low-vascularization regime, self-similar evolution to a steady state does not occur under these conditions. Similarly, self-similar unbounded growth in the high- or moderate-vascularization regimes does not occur. Further examinations [59] reveal the possibility of self-similar evolution to a steady-state in the low-vascularization regime and of unbounded selfsimilar growth in the high vascularization regime by varying $G$ in addition to A).

\section{Effect of Nonlinearity}

We investigate the effect of nonlinearity on the self-similar evolution for $d=2$ predicted by the linear analysis. As discussed above, self-similar evolution requires time-dependent apoptosis parameter $A=A(l, G, R)$ and plotted in Fig. 5 (top). Radius $R$, used in the nonlinear simulation, is determined by area of an equivalent circle: $R=\sqrt{H / \pi}$. In Fig. 6, the linear and nonlinear solutions are compared in the low-vascularization regime for $l=5, G=1$, $A=A(l, G, R)$ and $R_{0}=4$. Since $V<0$, the tumors shrink and $A$ increases. In the left frame, $\delta_{0}=0.2$ and in the bottom $\delta_{0}=0.4$. Results reveal that large perturbations are nonlinearly unstable and grow, leading to a topological transition. In the right frame, the onset of pinch-off is evident. This can have important implications for therapy. For example, one can imagine an experiment in which a tumor is made to shrink by therapy such that $A$ is increased by increasing the apoptosis rate $\lambda_{A}$. This example shows that a rapid decrease in size can result in shape instability leading to tumor break-up and the formation of microscopic tumor fragments that can enter the blood stream through leaky blood vessels thus leading to metastases.

\section{Evolution in the High Vascularization Regime}

In the high-vascularization regime $(G<0)$, both shrinkage and growth of tumors occur. Shrinkage $(A<0)$ may be stable, self-similar and unstable. In contrast, unbounded growth $(A>0)$ is always characterized by a decay 
of the perturbation to zero with respect to the unperturbed radius and is thus stable for both $d=2$ and $d=3$. In the nonlinear regime, self-similar and unstable shrinkage are qualitatively very similar to that presented in Fig. 6. In fact, all the nonlinear simulations of growth in the high vascularization regime lead to stable evolution, in agreement with the linear analysis. In contrast, it is known experimentally that highly vascularized cancers evolve invasively by extending branches into regions of the external tissue where mechanical resistance is lowest (e.g. [46]). Thus these results suggest that formation of invasive tumors should be due to anisotropies rather than to vascularization alone. Anisotropies (e.g., in the distribution of the resistance of the external tissue to tumor growth, or in the distribution of blood vessels) have been neglected in the model studied here. This conclusion has not been recognized before and is supported by experiments [151] of in vivo angiogenesis and tumor growth.

\subsection{Summary}

This work $[59,127]$ studied solid tumor growth in the nonlinear regime using boundary-integral simulations. In the model investigated [90, 142, 40, 41], the tumor core is assumed to be non-necrotic and no inhibitor factors to be present. A new formulation of this classical model was developed, and it was demonstrated that tumor evolution can be described by a reduced set of two dimensionless parameters and is qualitatively unaffected by the number of spatial dimensions. By constructing explicit examples using nonlinear simulations, it was demonstrated that critical conditions exist for which the tumor evolves to nontrivial dormant states or grows self-similarly. Self-similar growth separates stable tumors that grow maintaining a compact shape from unstable tumors, for which vascularization is favored and growth leads to invasive fingering into the healthy tissues. The possibility of tumor shape control during growth is suggested [59] by simulating a physical experiment in which the dimensionless parameters are varied in time to maintain stable or self-similar growth conditions thus preventing invasive growth and hampering angiogenesis. It can also be shown that nonlinear unstable growth may lead to topological transitions such as tumor breakup and reconnection with encapsulation of healthy tissue [59]. The boundary integral methods reviewed here provide evidence that morphologic instability represents an additional means for tumor invasion. 


\section{Vascularized Tumor Growth Using an Adaptive Finite-Element/Level-Set Method ${ }^{\S}$}

\subsection{Overview}

In [212] we use a reformulation of several well-known continuum-scale models of growth and angiogenesis $([90,38,39,46,11,80,25,16]$. We build upon the biological complexity of previous models that used boundary integral methods $[59,127]$ (described in Section 1.0), where tissue is represented as a constant density fluid whose elasticity is neglected. Some cell-cell adhesion is modeled using a surface force at the tumor interface. A single vital nutrient is modeled, e.g., oxygen or glucose that is required for cell survival and mitosis. Nutrient diffuses through the extracellular matrix (ECM) and is uptaken by tumor cells. Growth in this case is diffusion limited and necrosis may occur, unlike the methods in Sect. 1. Necrosis is represented as the region where nutrient is depleted below a certain level needed for cell viability. Once necrosis forms, the tumor releases tumor angiogenic factors (TAF), such as vascular endothelial growth factor (VEGF), which stimulate vascular endothelial cells to migrate toward the tumor. TAF is described through a reaction-diffusion equation with a point boundary condition set on the necrotic rim, modeling the release of TAF. Endothelial cells' density (ECD) obeys a reaction-diffusion-convection equation. The problem is convection-dominated, with primary source of convection driven by chemotaxis of endothelial cells with respect to TAF concentration. Actual formation of capillaries from endothelial cells is modeled using a combined continuum-discrete model [11], coupling the vascular system with tumor growth for the first time.

This combined continuum/discrete-scale model demonstrates the capabilities for nonlinear simulation of in vivo cancer progression in two dimensions, for which state-of-the art numerical methods based on sophisticated finite element techniques are necessary. The level set method [154] is employed for capturing invasive fingering and complex topological changes such as tumor splitting and reconnection, and healthy tissue capture. An adaptive, unstructured mesh [54] is used that allows for finely resolving important regions of the computational domain such as the necrotic rim, the tumor interface and around capillary sprouts. The adaptive mesh provides for enormous computational savings by greatly reducing the number of finite elements needed to resolve the finest length scale, when compared to a structured mesh having equal resolution.

\footnotetext{
$\S$ Portions of this section are reprinted with permission from Bull. Math. Biol., Zheng et al. Vol. 67, pp. 211-259, Copyright 2005 Springer (with kind permission of Springer Science and Business Media).
} 


\subsection{Dimensional formulation of the model}

The growth component of the model is inspired by the work of $[90,38,39,46$, $80,59]$. The angiogenesis model is essentially that of [11] with some additions gleaned from $[52,159,157]$. Figure 7 gives a basic description of these components, oversimplifying the underlying tumor physiology. The growth block is composed of nutrient transport, cell proliferation, and a constitutive equation that relates interstitial pressure and cell velocity. The angiogenesis block is composed of endothelial cell, fibronectin, and tumor angiogenic factor (TAF) transport equations. The schematic also illustrates how the two blocks are coupled throughout the evolution of a solid tumor. The instantaneous position of the necrotic rim $\Sigma_{N}(t)$ is determined by solving the growth problem and becomes an input to the angiogenesis problem by acting as a source of angiogenic factors.

Transfer of nutrients from capillaries to the tissue (characterized by the rate $\bar{T}_{c}$ ) depends on the instantaneous spatial distribution of capillaries determined by solving the angiogenesis problem and is an input to the growth block by acting as a source of nutrients. Rates (inverse time scales) are denoted by lowercase Greek letters with the following subscripts: (A) Apoptosis, (D) natural Degradation, (M) Mitosis, (N) Necrosis, (P) Production, or (U) Uptake. An overbar on a symbol indicates a dimensional parameter; the absence of an overbar indicates the corresponding nondimensional parameter. The field variables are

- concentration of tumor angiogenic factor (TAF) $c$

- endothelial cell's density (ECD) $e$

- density of fibronectin $f$

- concentration of (vital) nutrient $n$

- pressure $p$

- velocity $\mathbf{u}$

Both dimensional and nondimensional fields are expressed by the same symbol; the difference will be clear from the context.

\section{Tumor Growth}

Consider a computational tissue domain $\Omega$ that contains three disjoint subdomains, viable tumor $\Omega_{V}$, necrotic core $\Omega_{N}$, and external healthy tissue $\Omega_{H}$ (cf. Fig 8). The necrotic core contains cells that are not viable due to a lack of available nutrients and is defined as $\Omega_{N}=\left\{\mathbf{x} \mid \mathbf{n}(\mathbf{x}) \leq \overline{\mathbf{n}}_{\mathbf{N}}\right\} \backslash \Omega_{H}$, where $\bar{n}_{N}$ is the minimum nutrient level for cell viability. For simplicity, healthy cells are assumed to remain viable even when nutrient falls below $\bar{n}_{N}$. The tumor domain, which is defined as $\Omega_{T}=\Omega_{V} \cup \Omega_{N}$, contains both viable and necrotic cells. The nutrient concentration $n$ obeys the following: 


$$
0=\bar{D}_{n} \nabla^{2} n+ \begin{cases}\bar{T}_{c}-\bar{\nu}_{U} n & \text { if } x \in \Omega_{V} \\ \bar{T}_{c} & \text { if } x \in \Omega_{H} \\ 0 & \text { if } x \in \Omega_{N}\end{cases}
$$

where $\bar{D}_{n}$ is a diffusion constant, $\bar{\nu}_{U}$ is nutrient consumption rate by the tumor cells, and $\bar{T}_{c}$ is the capillary-to-tissue nutrient transfer function. There is assumed no transfer of nutrients in the necrotic core. $\bar{T}_{c}$ may vary depending on the differences in nutrient and pressure levels between the capillaries and the surrounding tissue. We use the simple model $\bar{T}_{c}=\bar{T}_{c}^{\mathrm{ang}}+\bar{T}_{c}^{\mathrm{pre}}$ where

$$
\begin{gathered}
\bar{T}_{c}^{\mathrm{ang}}=\bar{\nu}_{p}^{\mathrm{ang}}\left(\bar{n}_{c}-n\right) b^{\mathrm{ang}}(p) \delta_{c} \\
\bar{T}_{c}^{\mathrm{pre}}=\bar{\nu}_{p}^{\mathrm{pre}}\left(\bar{n}_{c}-n\right) b^{\mathrm{pre}}(p)
\end{gathered}
$$

where 'ang' and 'pre' denote new capillaries formed during angiogenesis and the uniformly distributed, preexisting capillaries, respectively. The $\bar{\nu}_{p}^{*}\left(^{*}=\right.$ ang, pre) are nutrient transfer (production) rates for new and old capillaries, $\bar{n}_{c}$ is nutrient concentration in capillaries, and $p$ is tissue pressure. Pressure functions $b^{*}(p)$ have the form $b^{*}(p)=H\left(\bar{p}_{c}^{*}-p\right)\left(\bar{p}_{c}^{*}-p\right) / \bar{p}_{T}$, where $\bar{p}_{c}^{*}$ are new and preexisting capillary blood pressures, $\bar{p}_{T}$ is a characteristic tissue pressure. $\delta_{C}$ is a dimensionless line delta function supported on the line domain $\Sigma_{C}$, where $\Sigma_{C}$ describes the positions of all those capillaries that have formed loops in the new vasculature, hereby assuming that effective blood flow only occurs in capillaries that have formed loops (see Fig 8). Integrating (17) gives line-sources of nutrients emanating from the looped capillaries.

The nutrient concentration is fixed at the outer (far field) boundary $\Sigma_{\infty}$, is continuous (up to the first derivative) at interface $\Sigma$ between the tumor and healthy tissue domains and at the necrotic interface $\Sigma_{N}=$ $\left\{\mathbf{x} \mid \mathbf{n}(\mathbf{x})=\overline{\mathbf{n}}_{\mathbf{N}}\right\} \backslash \Omega_{H}$ between necrotic core and healthy tissue:

$$
\begin{gathered}
n=\bar{n}_{\infty} \quad \text { on } \Sigma_{\infty} \\
{[n]=0, \quad[\zeta \cdot \nabla n]=0} \\
n=\bar{n}_{N}, \quad\left[\zeta_{N} \cdot \nabla n\right]=0 \quad \text { on } \Sigma \\
\end{gathered}
$$

where $\zeta$ and $\zeta_{N}$ are unit normal vectors on $\Sigma$ and $\Sigma_{N}$, respectively and [.] denotes the jump of the quantity across the interface (value inside minus value outside). Tumor cell proliferation leads to motion of the cells and growth of the overall tumor. The divergence of the cell velocity field obeys equations 


$$
\nabla \cdot \mathbf{u}= \begin{cases}\lambda_{M} n / \bar{n}_{\infty}-\bar{\lambda}_{A} & \text { if } \mathbf{x} \in \Omega_{V} \\ 0 & \text { if } \mathbf{x} \in \Omega_{H} \\ -\bar{\lambda}_{N} & \text { if } \mathbf{x} \in \Omega_{N}\end{cases}
$$

where cell mitosis is assumed proportional to local nutrient concentration and $\bar{\lambda}_{M}$ is characteristic tumor cell mitosis rate. Parameter $\bar{\lambda}_{A}$ is tumor cell apoptosis rate, and $\bar{\lambda}_{N}$ is a rate of volume loss in the necrotic core modeling cell necrosis and disintegration therein. Note that diffusion and chemotaxis of tumor cells is neglected, which have been taken into account in the model by [48], to emphasize tissue invasiondue to uncontrolled proliferation of tumor cells. It should be noted that diffusion of cells is orders of magnitude smaller than advection, and that extent of chemotactic response of tumor cells is not universally agreed upon. Tumor cells are modeled at the continuum level as a viscous fluid [e.g., see [46]] flowing through a porous medium (extracellular matrix). Accordingly, motion of tumor cells obeys Darcy-Stokes law [also called the Brinkman equation [196]: in $\Omega$ the velocity and pressure are related by

$$
\mathbf{u}-\bar{\varepsilon} \nabla^{\mathbf{2}} \mathbf{u}=-\bar{\mu} \nabla \mathbf{p}
$$

where $\bar{\varepsilon}$ is a constant and $\bar{\mu}$ is cell mobility. This is also called hydraulic conductivity [196] and represents in this case the extent of cell motion in response to a given pressure gradient, caused by nonhomogeneous cell proliferation. We introduce $\bar{\varepsilon} \nabla^{2} \mathbf{u}$ as a regularization term for the velocity and pressure and assume that $\bar{\varepsilon}$ is sufficiently small. This term helps keep the numerical scheme stable, especially with regard to the velocity calculation at the tumor interface. However, (22) can be regarded as physical (Batchelor, 1967, Chapter $3[21]$ ) - whence $\bar{\varepsilon}$ becomes related to viscous stress associated to cell motion-upon introducing the physical pressure $P$, which is related to calculated (regularized) pressure $p$ through $p=P-\frac{\bar{\varepsilon}}{3 \bar{\mu}} \nabla \cdot \mathbf{u}$.

Velocity is assumed continuous across the necrotic rim, $\Sigma_{N}$, and tumor boundary $\Sigma$ :

$$
[\mathbf{u}]=\mathbf{0} \text { on } \boldsymbol{\Sigma}_{\mathbf{N}},[\mathbf{u}]=\mathbf{0} \text { on } \boldsymbol{\Sigma}
$$

A regularized Laplace-Young jump condition is assumed at the tumor interface $\Sigma$ to model cell-cell adhesion:

$$
\left(\left(-p \mathbf{I}+\frac{\bar{\varepsilon}}{\bar{\mu}} \nabla \mathbf{u}\right) \cdot \zeta\right)=-\bar{\gamma} \kappa \zeta \text { on } \boldsymbol{\Sigma}
$$

where $\bar{\gamma}$ is surface tension related to cell-to-cell adhesive forces, and $\kappa$ is local total curvature. Note that in this case the pressure may still experience a jump at the boundary when $\bar{\gamma}=0$ and $\bar{\varepsilon} \neq 0$. At the outer boundary $\Sigma_{\infty}$ we enforce the natural boundary condition:

$$
\left(-p \mathbf{I}+\frac{\bar{\varepsilon}}{\bar{\mu}} \nabla \mathbf{u}\right) \cdot \zeta_{\infty}=\mathbf{0} \text { on } \boldsymbol{\Sigma}_{\infty}
$$




\section{Angiogenesis}

Once a tumor cell senses that the nutrient level has dropped below the minimum for viability, the cell releases tumor angiogenic factors (TAF) that diffuse throughout the extracellular space. Endothelial cells become activated and migrate through the extracellular matrix towards the highest concentration of TAF at the necrotic rim of the tumor. As cell clusters move towards the tumor, the vasculature extends, using endothelial cells to construct the lining of capillaries (endothelium). This process is illustrated in Fig. 8. TAF molecules are much smaller than cells and diffuse quickly through the extracellular spaces. The quasi-steady reaction-diffusion equation is assumed for the concentration $c(\mathbf{x}, t)$ of TAF in $\Omega_{H}$ and $\Omega_{V}$

$$
0=\bar{D}_{c} \nabla^{2} c-\bar{\beta}_{D} c-\bar{\beta}_{U} c e / \bar{e}_{0}
$$

where $\bar{D}_{c}$ is diffusion constant, $\bar{\beta}_{D}$ is rate of natural decay of TAF, $\bar{\beta}_{U}$ is rate of uptake of TAF by endothelial cells (of density $e$ ), and $\bar{e}_{0}$ is maximum density of endothelial cells in $\Omega$. Here, as a simplifying step and to reduce unknowns, parameters in the last equation are assumed homogeneous, which is in general not valid. In particular, diffusion can be different inside the tumor from outside, as the diffusivity should depend upon parameters such as cellular density of tumor and healthy tissues.

Concentration of TAF is constant at the necrotic interface, modeling release of TAF by tumor cells as they undergo necrosis

$$
c=\bar{c}_{0} \text { on } \Sigma_{N}
$$

No-flux boundary conditions are imposed on $\Sigma_{\infty}$ :

$$
\zeta_{\infty} \cdot \nabla c=0
$$

where $\zeta_{\infty}$ is the surface unit normal on $\Sigma_{\infty}$.

A primary component of the extracellular matrix is fibronectin, a long binding molecule that does not diffuse. Endothelial cells produce, degrade, and attach to these molecules during their migration toward the tumor. The concentration of fibronectin $f(\mathbf{x}, t)$ obeys

$$
\frac{\partial f}{\partial t}=\bar{\eta}_{p} \bar{f}_{0} e / \bar{e}_{0}-\bar{\eta}_{U} f e / \bar{e}_{0}-\bar{\eta}_{N} \chi_{\Omega_{N}} f
$$

where $\bar{\eta}_{p}$ is rate of production of fibronectin by endothelial cells and $\bar{\eta}_{U}$ is rate of degradation of fibronectin by endothelial cells. Degradation of fibronectin is modeled due to the presence of the necrotic core, where $\bar{\eta}_{N}$ is rate of decay in the necrotic core. The initial condition for concentration of fibronectin is taken as $f(\mathbf{x}, 0)=\bar{f}_{0}$, where $\bar{f}_{0}$ is fibronectin concentration in healthy tissue. $\chi_{S}$ is the characteristic function of the set $S: \chi_{S}(\mathbf{x})=1$ if $\mathbf{x} \in S$ and vanishes otherwise. 
Endothelial cells are comparable in size to healthy and tumor cells. However, we assume that the former are few enough in number that the interaction of the former does not affect the calculation of the cell velocity $\mathbf{u}$. Indeed, the ratio of endothelial to tissue cells is of the order $1 / 50$ or $1 / 100$ [35]. On the other hand, the motion of healthy and tumor cells is an important consideration on convection of endothelial cells. Density $e(\mathbf{x}, t)$ of endothelial cells (related to the probability to find the tip of a capillary at that location and time) obeys the convection-reaction-diffusion equation in $\Omega_{H}$ and $\Omega_{V}$

$$
\begin{aligned}
\frac{\partial e}{\partial t} & =\bar{D}_{e} \nabla^{2} e-\nabla \cdot\left(\left(\frac{\chi_{C}}{1+\alpha c / \bar{c}_{0}} \nabla c+\bar{\chi}_{f} \nabla f+\chi_{\mathbf{u}} \mathbf{u}\right) e\right) \\
& -\bar{\rho}_{D} e+\bar{\rho}_{p} \frac{e\left(\bar{e}_{0}-e\right)}{\bar{e}_{0}} \mathcal{H}\left(c-\bar{c}^{*}\right) \frac{\left(c-\bar{c}^{*}\right)}{\bar{c}_{0}}-\rho_{N} \chi_{\Omega_{N}} e
\end{aligned}
$$

where $\bar{D}_{e}$ is diffusion constant, $\bar{\chi}_{c}, \bar{\chi}_{f}$ are chemotaxis and haptotaxis coefficients, respectively, and $\bar{\chi}_{\mathbf{u}}$ and $\alpha$ are dimensionless constants (herein, take $\chi_{\mathbf{u}}=0.2$ and $\alpha=0.1$ ). As assumed in [11], diffusion is dominated by convection. $\bar{c}^{*}$ is a concentration of TAF above which proliferation occurs. $\bar{\rho}_{D}, \bar{\rho}_{p}$, and $\bar{\rho}_{N}$ are rates of natural degradation, production and necrosis of endothelial cells, respectively. Initial condition for $e$ is

$$
e(\mathbf{x}, 0)=\bar{e}_{0} \sum_{i=1}^{m} \exp \left(-r_{i}^{2} / s_{e}^{2}\right)
$$

where $m$ is number of initial capillary sprout-tips, $r_{i}$ is distance from the $i$ th spout tip, and $s_{e}$ is a distance measuring spread of initial endothelial cell clusters. Boundary condition for $e(\mathbf{x}, t)$ is

$$
\zeta_{\infty} \cdot\left(\overline{\mathbf{V}} e-\bar{D}_{e} \nabla e\right)=0 \text { on } \Sigma_{\infty}
$$

where $\zeta_{\infty}$ is the unit normal vector, and $\overline{\mathbf{V}}$ is the full convective velocity:

$$
\overline{\mathbf{V}}=\frac{\bar{\chi}_{C}}{1+\alpha c / \bar{c}_{0}} \nabla c+\bar{\chi}_{f} \nabla f+\chi_{\mathbf{u}} \mathbf{u}
$$

The model for motion of capillary sprout-tips is comprised of continuum and discrete components [11]. Equations (26), (29) and (30) constitute the continuum component. The discrete component is founded on the assumption that growth of the capillary is simply determined by the biased random migration (random walk) of a single endothelial cell at the sprout-tip. Given the time invariant spatial step size $h_{\mathrm{reg}}$, and continuum fields $c\left(\mathbf{x}, t_{0}\right)$ and $f\left(\mathbf{x}, t_{0}\right)$, the model predicts the probabilities that at time $t_{0}+\Delta t$ the single cell stays at $\mathbf{x}$ or moves to one of four nearest neighbor sites, $\mathbf{x} \pm h_{\mathrm{reg}} \mathbf{e}_{i}$, where $\mathbf{e}_{i}$ are the canonical orthonormal basis vectors. These probabilities are calculated by finite-differencing (30) following the method of [11]. The algorithm then updates the cell's position by a weighted random selection based on the five 
probabilities. Besides tip migration, the discrete-continuum scheme can predict capillary branching and anastomosis (fusion), and recently this framework has been used to include flow and the delivery of nutrients and drugs [141]. Unlike [11] or [141], we allow for the possibility that the entire capillary may be convected by the external cell velocity using the kinematic condition

$$
\frac{d \mathbf{x}}{d t}=\mu_{C} \mathbf{u}
$$

where $\mathbf{x}$ is the position on the capillary and $\mu_{C}$ is to be interpreted as the mobility of the capillary (herein use the small value $\mu_{C}=0.1$ ). This is a simplified model for the vasculature treating all points on the vessels the same. In fact, as the network becomes more established in time the capillaries become harder to move, which is not accounted for here. Also, the precise characteristics of flow in the capillary network are not considered, except for accounting for the pressure effect on flow according to (18).

\subsection{Results}

The reaction-diffusion equations in the model outlined above are solved using an adaptive $[54,9]$ finite-element/level-set method in two spatial dimensions. We present a simulation using the combined growth and angiogenesis models. Complete model parameters are given in Figure 9. Computational domain is $\Omega=(-40,40) \times(-40,40)$. Details of the simulation for nondimensional times $t=0$ to 900 are displayed in Fig. 10 (only part of the domain is shown). Left column shows current shape of the tumor interface (thick solid line), location of the necrotic rim (thin), and new capillaries (solid branching and looping lines) and contours of ECD (dashed). Plots of fibronectin concentration (FIB), nutrient concentration $(\mathrm{N})$, pressure $(\mathrm{P})$, endothelial cell density $(\mathrm{ECD})$, and tumor angiogenic factor (TAF) concentration are also calculated; nutrient is shown in the right column. The tumor is initially centered at the origin with its interface given by

$$
(x(\theta), y(\theta))=(2+0.1 \cos (2 \theta))(\cos (\theta), \sin (\theta))
$$

where $\theta$ is the angle measured counterclockwise off of the $x$-axis. The tumor is surrounded by six preselected capillary sprouts, which provide initial conditions for the free endothelial cell clusters. To accentuate the effect of angiogenesis, the transfer of nutrients from the uniform vasculature is assumed negligible in the near environment of the tumor $\left(v_{p}^{\text {pre }}=0\right)$, and the nutrient is assumed to come only from the outside environment $\left(n=1\right.$ on $\left.\Sigma_{\infty}\right)$ and from the neo-vasculature.

\section{Microphysical Parameters for Malignant Glioma}

Input parameters were selected based on the following considerations. In vivo, for example, malignant glioma (brain tumor), apoptosis is probably negligible 
$\left(\lambda_{A}=0\right)$ since mutated clones after the initial selection has occurred are typically characterized by suppression of the p 53 pathway and enhanced proliferation [137], and even normal glial cells are immortal. From experiments [74] on growth of glioblastoma cell lines (the most malignant and proliferative brain tumors) as multicellular millimeter-size spheroids in vitro, it was observed that typically mitosis rate $\bar{\lambda}_{M} \approx 0.3$ day $^{-1}$, that rate of volume loss in the necrotic core is comparable or less than the rate of volume gain from mitosis, hence the choice $\lambda_{N}=0.25$, and finally that minimum nutrient concentration for cell viability is a small fraction of outer uniform concentration in growth medium [74], hence the choice of a value for $n_{N} \ll 1$ (although the value of this parameter may be affected by the difficulty of reproducing the in vivo conditions in vitro). Dimensionless nutrient concentration in the blood is chosen as $n_{C}=1$, i.e., in equilibrium with the concentration in the undisturbed tissue environment. Nutrient transfer rate $v_{p}^{\text {ang }}$ is set after some experimentation to a value that provides a continuous supply of nutrient during the tumor's entire evolution, in order to reproduce in vivo growth and infiltration of neo-vascularized glioblastoma [137]. A value for the pressure is chosen as $p_{C}^{\text {ang }}=O(1)$ in the capillaries, thus in near equilibrium with interstitial pressure in glioma. By considering a characteristic diffusion constant for nutrient $\bar{D}_{n} \approx 10^{-5} \mathrm{~cm}^{2} / s$ and a nutrient consumption rate $v_{U} \approx 1 \mathrm{~min}^{-1}$ (the latter from the observation that brain cells run out of glucose and die on that time scale), a characteristic nutrient diffusion length $\mathcal{L} \approx 200-300 \mu \mathrm{m}$ is obtained. This is consistent with the observed thickness of the viable rim of cells in tumor spheroids in vitro $[2,74]$. For the simulation illustrated in Fig. 10, the tumor grows to a size $\approx 40 \cdot \mathcal{L} \approx 8 \mathrm{~mm}-1.2 \mathrm{~cm}$ on a time scale $\approx 900 \lambda_{M}^{-1} \approx 7$ years which is in quite good agreement with the observed time of growth of high grade malignant glioma (astrocytoma) to secondary glioblastoma [137]. The surface tension parameter was chosen at a value small enough that cell adhesive forces are weak and diffusional instability of the tumor shape occurs during growth as observed in the experiments [74]. Angiogenesis parameters were chosen to obtain continuous growth of neo-vasculature parallel to growth of the tumor as is observed clinically. The dimensionless diffusion constant of TAF was chosen to ensure a nonzero value of TAF concentration over the length scale of the tumor size. Rates characterizing fibronectin evolution were chosen to ensure an observable effect of haptotaxis (responsible for looping of the neo-capillaries).

\section{Diffusional Instability and Tissue Invasion}

For the parameters given in Fig. 9 and an initial, noncircular shape (35), the tumor will experience unstable growth due to a diffusional instability [36] caused by the competition of growth of the tumor mass and surface tension (cell adhesive forces) that tends to influence this growth. Note that without angiogenesis, and with a circular initial shape, instabilities would grow only 
due to random numerical error or because of the nonradially symmetric nutrient distribution due to the use of a square computational domain. The instability is enhanced by the development of a necrotic core and its associated volume sink. The tumor forms bulbs and breaks up into fragments. Indeed, the beginning of instability can be seen at time $t=200$ (Fig. 10b). The presence of the inhomogeneous nutrient field due to angiogenesis tends to further destabilize the tumor, because the tumor tends to co-opt the complexly shaped neo-vasculature in order to maximize nutrient transfer. Note that the resulting shape of the tumor is therefore stable to random noise or small perturbations because the patterns of growth of the tumor mass are mainly driven by the spatial distribution of the newly formed capillaries. By $t=400$ the tumor breaks up into three parts, as fragments of the tumor 'move' in opposite directions up the gradient of the nutrient concentration field (Fig. 10c). This net migration is due to the combination of cell death in low nutrient concentration and cell birth in high nutrient concentration. Note that in the simulations, this migration is exaggerated because we use a simplified model (Darcy's law) for the mechanical response of the tissue.

At the same time the formation of necrosis leads to release of tumor angiogenic factors (TAF) and thus triggers chemotaxis of endothelial cells and new capillary formation and migration towards the tumor from the preexisting blood vessels. At time $t=400$ penetration of the capillaries into the tumor has occurred (Fig. 10b). It is assumed that the same model describes angiogenesis outside and inside the tumor, which implies that an extracellular matrix (ECM) exists inside the tumor thus allowing chemotaxis and haptotaxis of endothelial cells to occur. This is probably a valid assumption for the case of malignant glioma, known to infiltrate through the brain ECM and co-exist there with healthy cells [137]. Note also that we assume infinitesimal thickness of capillaries and no mechanical interaction with the tissue (except for the small mobility $\mu_{C}$ ). Thus here capillaries are line sources of nutrient. As the disjoint tumor fragments continue to grow, the tumor reconnects $(700<t<800)$. Note also the small fragment in the middle $(t=400)$ later shrinks and disappears. By time $t=900$ (Fig. 10d), the tumor has almost completely co-opted the neo-vasculature. It has been hypothesized that tissue invasion occurs in cycles. Within one cycle, angiogenesis occurs followed by tumor mass growth and co-option of the newly formed vessels. These cycles would repeat themselves. Here, one of these cycles is simulated. Further growth would require further vascularization. The complex tumor morphologies predicted in this simulation are ultimately due to the nonuniform distribution of nutrient sources following angiogenesis. The capability of simulating the coupled growth and angiogenesis processes leads to predictions of tumor morphologies that are more realistic than those predicted in previous investigations (Sect. 1).

By $t=900$ two smaller tumor clusters have fragmented-off because of diffusional instability and are migrating towards the computational boundary $\Sigma_{\infty}$, where the nutrient level is highest (bottom of Fig. 10d), and a third will 
fragment-off shortly. If the surface tension $\gamma$ were larger, the tumor shape would remain compact, and fragmentation would not occur. Here low surface tension (and cell mobility) models weak adhesive forces that enable cell clusters to scatter and migrate and infiltrate through the extracellular matrix. In malignant glioma, weak cell adhesion is probably the effect of excitation of the FAK (focal adhesion kinase) pathways [149] that are triggered by overexpression of EGFR (epidermal growth factor receptor) and its numerous ligands on the tumor cell surfaces. EGFR signaling activates both MAPK proliferative pathways and FAK cell-scattering pathways [149]. In this simulation, fragment migration occurs in a manner similar to the migration of water droplets in ice in the presence of a temperature gradient [195] due to a melt-and-freeze mechanism. Proliferation occurs near the leading edge of the fragment, whereas necrosis occurs at the trailing edge, thus leading to a net migration of cells up the nutrient concentration levels (Fig. 11, left). This can be observed in the pressure field around the migrating fragments at time $t=900$ (pressure (P) in Fig. 11, right). The pressure is higher at the leading edge, and lower at the trailing edge. In fact there is a mass sink at the trailing edge owing to the necrotic core. This provides insight into a possible mechanism for tissue invasionby tumor cell clusters [76], especially in brain tumors where the cancer cells are observed to powerfully infiltrate the brain's extracellular matrix. In addition there is migration due to gradients of adhesiveness, which is neglected in this model, but considered by [48]. Finally, here tumor cell apoptosis is neglected. However, if $\lambda_{A}>0$, the same net migration is observed in the simulations without the presence of a necrotic core, in agreement with the infiltrative nature of low- and high-grade astrocytoma that do not exhibit necrosis [137].

By multiplying the dimensionless tumor area at $t=900$ by $\mathcal{L}^{2}$ with $\mathcal{L}=$ $300 \mu \mathrm{m}$, and the dimensionless capillary length by $\mathcal{L}$, and then by scaling the two dimensional simulation to a three-dimensional tumor using an exponent $3 / 2$, one obtains a total predicted tumor volume $\approx 1 \mathrm{~cm}^{3}$ and, by assuming an equivalent volume per cell of $1000 \mu \mathrm{m}^{3}$, a prediction of $\approx 1$ billion tumor cells and a ratio of tumor-to-capillary cells $\approx 61$, in good agreement with experimental observations [35].

\section{Nutrient Transfer from the Neo-vasculature}

Supply of nutrients is nonuniform and nonconstant in time in this model, owing to the extension of the capillaries to the tumor. This extension is a continuous feedback process because angiogenesis (i) occurs as a response to diminishing nutrient levels in the tumor and (ii) raises the nutrient supply in the tumor allowing for increased growth and increased nutrient demand. In addition there is a stabilizing effect of pressure in the nutrient transfer [(18)]. In the initial stages of neo-vascularization when the amount of nutrient coming from the new capillaries is low compared to that of other sources, the angiogenesis process is decoupled from growth, as in [11]. This is the case up 
to approximately time $t=200$ (Fig. 10b). Note that the diffusional shape instability is not due to angiogenesis. After this point, the nutrient supply coming from the new capillaries is important for the growth of the tumor. By time $t=400$ the capillaries penetrate the tumor. Splitting (branching) and looping (anastomosis) are also observed. In our implementation nutrients are transferred only from looped vessels, since these vessels can provide a more effective flow of blood. One can effectively control the density of looped vessels by adjusting the density of new capillaries through the splitting probability and the looping criteria through the minimum age for looping and the effective loop-receptive regions on the capillaries [11]. Subsequent to penetration, the tumor begins to co-opt the new capillaries. This process occurs because the tumor grows more near the (looped) capillaries. In this simulation (Fig. 10d), the tumor takes on the shape of the new vasculature, roughly. A recent hypothesis [137] for the infiltration of malignant glioma cells throughout the brain is that these cells co-opt and crawl around the preexisting brain vasculature. The behavior observed in the simulation seems to corroborate this hypothesis by providing an additional mechanism for infiltration.

In the present simulation, growth of the vasculature slows dramatically after about time $t=400$ (and the capillaries are only advected by the growing tumor according to (34) thereafter). In the model, a minimum amount of ECD is required for the continued growth of the sprout tips. Here, the amount of endothelial cell proliferation is smaller than degradation and necrosis, and the amount of endothelial cells decreases. However, with sufficient proliferation, the neovascularization can even speed-up, since the rate of capillary splitting generally depends on local levels of ECD [11]. Although only six activated sprout tips are included, one may also increase this number initially, or stochastically add more sites as the tumor grows, with activation probabilities depending upon the local TAF levels. In the simulation in Fig. 10, local levels of TAF generally increase along the radial direction as the tumor grows. Hence more active sprout tip sites are activated in time.

If suddenly the nutrient concentration became depleted, or the flow of nutrients were cut off ( $n=0$ everywhere in $\Omega$ ) the tumor would shrink and vanish, owing to the death term $\lambda_{N}$ in (21). If instead only the flow from the neo-vasculature were cut off, with the far field nutrient source in place ( $n=1$ on $\Sigma_{\infty}$ ), the tumor would shrink to a diffusion-limited, stationary size. Additionally, the growth of the vascularized tumor depends vitally on the nutrient transfer rate $\nu_{p}^{\text {ang }}$, the level of nutrient in the blood $n_{C}$, and the blood pressure in the new capillaries $p_{C}^{\text {ang }}$. Here we have taken the blood pressure to be $p_{C}^{\text {ang }}=1$, i.e., equal to the characteristic pressure due to tumor cell mitosis. Because of the form of $b^{\text {ang }}(p)$, there is no nutrient transfer in an environment for which $p>1$. This restricts growth by providing a stabilizing mechanism because the tumor must balance the rate of growth with pressure level its growth causes. The result is that total pressure tends to be bounded away from $p_{C}^{\text {ang }}$. Increasing pressure in capillaries increases the rate of nutrient 
transfer, and hence the rate of growth. But again the pressure will be bounded away from $p_{C}^{\text {ang }}$. In vivo this pressure is responsible for the difficulties in delivering chemotherapeutic agents through the vasculature to tumor cells. The simulations in Figs. 10c and 10d reveal that inside the tumor the transfer from the vasculature nearly vanishes. As a result of these phenomena the tumor configuration at time $t=900$ is nearly stationary; further growth will require an ongoing angiogenesis process. Finally note that the relatively small amount of necrosis in the last frame is an artifact of the model used, which implements a very sharp transition from live to necrotic cells at $n=n_{N}$. In reality, the transition is smoother and in the tumor body depicted in the last frame $(t=900)$ necrotic and nearly necrotic cells are mixed over a larger region.

\subsection{Summary}

The work presented here displays at least two new, important features vital for the development of a realistic virtual cancer simulator at the tumoral level. First, it successfully incorporates a realistic model of angiogenesis [11] with a continuum model for growth. Previous models for growth have only included constant vascularization [59] or a field variable representing spatially smooth vascularization [32]. In the model reviewed here, the capillary network extends due to direct interaction with the growing tumor, primarily through the TAF. At the same time, the tumor receives nutrient, by bloodto-tissue transfer from the extending capillary network. Second, this work is fully two-dimensional, incorporating more realistic tumor evolution than can be realized in lower-dimensional (e.g., spherically symmetric, cylindrical or one-dimensional) models of growth and angiogenesis. We used sophisticated numerical techniques to accomplish this: the level set method [154] for capturing complicated morphology and connectedness of the tumor interface, and finite element methods based on a fully-adaptive unstructured mesh for accurately resolving multiple length scales at the lowest possible computational expense. Such efforts enable the simulator to model phenomena such as invasive fingering, tumor fragmentation, and healthy tissue capture. This work significantly extends that of Sect. 1, which used a simpler model for growth and vascularization and the boundary integral method for two-dimensional computation.

Our simulation results are supported by experimental data. In a parallel study [74], we used the grade IV glioma (glioblastoma multiforme) human cell line ACBT to culture spheroids in vitro over several weeks and observe their growth pattern as a function of nutrient (glucose) and growth factor (serum) variation. At high serum concentrations, spheroids were observed to grow to a diffusion-limited millimeter size and then become unstable and assume dimpled shapes as in our simulation. These sub-spheroidal structures may eventually break off from the main spheroid (as a 'bubble' of cells or as spheroid fragments) or merge with neighboring structures to enlarge the 
overall parental spheroid mass. This process can repeat itself on the subspheroid, leading to recursive sub-spheroidal growth as the main mechanism of spheroid morphogenesis. The stability of the sub-spheroidal components is dependent on the rate of cell proliferation and death, as controlled by access to nutrient and growth factors. Our simulations and these experiments indicate that diffusional shape instability may be a powerful tissue invasion mechanism, since for the same mitosis and death rates spherical tumors would remain of millimeter size and never become clinically relevant. The results indicate that diffusional instability allows clusters of tumor cells to invade and infiltrate the surrounding brain even without resorting to further genetic alterations that differentiate these cells from the bulk tumor by enhancing their mobility. Note that it has been observed that cancer cell migration is different from model cells. It has been shown that tumor cells migrate in cell clusters rather than as single cells [76]. Note also that in malignant glioma genotypes, heterogeneity in the mutations of the focal adhesion kinase (FAK) pathways that regulate cell scattering and adhesion is rarely if ever observed [137], although this matter is still very unclear and caution should be used when drawing conclusions.

\section{Solid Tumor Growth Using a Ghost Cell/Level-Set Method ${ }^{\text {T }}$}

\subsection{Overview}

In $[135,136]$ we extended tumor growth models considered by $[59,133,212]$ and others, which reformulated classical models $[90,142,3,39,40,47]$ to include more detailed effects of the microenvironment. This was done by allowing variability in nutrient availability and the response to proliferationinduced mechanical pressure (which models hydrostatic stress) in the tissue surrounding the tumor. Using analysis and nonlinear numerical simulations, we explored the effects of the interaction between the genetic characteristics of the tumor and the tumor microenvironment on the resulting tumor progression and morphology. We found that the range of morphological responses can be placed in three categories that depend primarily upon the tumor microenvironment: tissue invasion via fragmentation due to a hypoxic microenvironment; fingering, invasive growth into nutrient rich, biomechanically unresponsive tissue; and compact growth into nutrient rich, biomechanically responsive tissue. The qualitative behavior of tumor morphologies was similar across a broad range of parameters that govern tumor genetic characteristics. These findings demonstrate the importance of the microenvironment on tumor growth and morphology and implications for therapy.

\footnotetext{
T This section includes an article published in J. Theor. Biol., Vol. 245, Macklin \& Lowengrub, Nonlinear simulation of the effect of microenvironment on tumor growth, pp. 677-704, Copyright (C) Elsevier (2007), and portions reprinted from Macklin \& Lowengrub (in review) [136].
} 
Previously $[132,133,134]$ we considered a level set-based extension of the tumor growth model by [59] (Sect. 1), where we developed new, highly accurate numerical techniques to solve the resulting system of partial differential equations in a moving domain. These methods are more accurate than those used by [212] (Sect. 2) and [97]. Using the new methods, we modeled tumor growth under a variety of conditions and investigated the role of necrosis in destabilizing the tumor morphology. We demonstrated that non-homogeneous nutrient diffusion inside a tumor leads to heterogeneous growth patterns that, when interacting with cell-cell adhesion, cause sustained morphological instability during tumor growth, as well as repeated encapsulation of non-cancerous tissue by a growing tumor. Building upon this earlier work, [136] developed an accurate ghost cell/level set technique for evolving interfaces whose normal velocity is given by normal derivatives of solutions to linear and nonlinear quasisteady reaction-diffusion equations with curvature-dependent boundary conditions. The technique is capable of describing complex morphologies evolving in heterogeneous domains. The algorithm involves several new developments, including a new ghost cell technique for accurately discretizing jumps in the normal derivative without smearing jumps in the tangential derivative, a new adaptive solver for linear and nonlinear quasi-steady reaction-diffusion problems (NAGSI), an adaptive normal vector discretization for interfaces in close contact, and an accurate discrete approximation to the Heaviside function.

In our model, the region surrounding a tumor aggregates the effects of ECM and non-cancerous cells, which are characterized by two non-dimensional parameters that govern diffusional and biomechanical properties of the tissue. Fluids are assumed to move freely through interstitium and ECM, and thus these effects are currently neglected. External nutrient and pressure variations, in turn, affect tumor evolution. Due to computational cost of 3-D simulations, we focus on 2-D tumor growth, although the model applies equally well in three dimensions. In [59] (Sect. 1), it was found that the baseline model predicts similar morphological behavior for 2-D and 3-D tumor growth. This has been borne out by recent 3-D simulations by [127], as seen in Sect. 1 . Note that 2-D tumor growth may be well suited to study cancers that spread relatively thinly, such as melanoma.

We also investigated internal structure of tumors, including volume fractions of necrotic and viable regions. We found that even during growth, the internal structure tends to stabilize due to apparent local equilibration of tumors as characteristic feature sizes and shapes emerge. Whereas tumor morphology depends primarily upon the microenvironment, internal structure is most strongly influenced by tumor genetic characteristics, including resistance to necrosis, the rate at which necrosis is degraded, and rate of apoptosis. These results are not obvious from examination of the model and underlying hypotheses alone. By hypothesis, the microenvironment, tumor genetics, and tumor morphology are all nonlinearly coupled. Tumor genetics determine biophysical properties like growth rates, which, in turn, are mediated by microenvironmental factors such as available nutrient supply. One would expect 
that tumor genetics have a greater impact on tumor morphology, and, indeed, [59] provides evidence that tumor genetics completely determine the morphological behavior when the microenvironment is not taken into account.

\subsection{Governing Equations}

We model an avascular tumor occupying volume $\Omega_{T}(t)$ with boundary $\partial \Omega$, denoted by $\Sigma$. The tumor is composed of a viable region $\Omega_{V}$ where nutrient (e.g., oxygen and glucose) levels are sufficient for tumor cell viability and a necrotic region $\Omega_{N}$ where tumor cells die due to low nutrient levels and are broken down. Note that $\Omega_{T}=\Omega_{V} \cup \Omega_{N}$. The growing tumor also interacts with the surrounding microenvironment in the host tissue; this region is denoted by $\Omega_{H}$, which contains ECM and a mixture of non-cancerous cells, fluid, and cellular debris. As observed in $[59,132,133]$, a growing tumor may encapsulate regions of $\Omega_{H}$, and so these regions may lack living noncancerous cells. Hereafter, we refer to $\Omega_{H}$ as non-cancerous tissue, although the model applies equally well to the case in which $\Omega_{H}$ contains only ECM, fluid, and cellular debris.

\section{Nutrient Transport}

As defined in Sect. 2, we set capillary-to-tissue nutrient transfer function $\bar{T}_{c}=$ 0 and use relative nutrient diffusivity $D$ to control level of nutrient in $\Omega_{H}$ : $\bar{D}_{n}=1$ in $\Omega_{T}$ and $\bar{D}_{n}=D$ in $\Omega_{H}$.

\section{Cellular Velocity Field}

Cells and ECMin host tissue $\Omega_{H}$ and viable tumor region $\Omega_{V}$ are affected by a variety of forces, each of which contributes to the cellular velocity field $\mathbf{u}$. Proliferating tumor cells in $\Omega_{V}$ generate an internal (oncotic) mechanical pressure (hydrostatic stress) that also exerts force on surrounding non-cancerous tissue in $\Omega_{H}$. Tumor and non-cancerous cells and ECM can respond to pressure variations by overcoming cell-cell and cell-ECM adhesion and moving within the scaffolding of collagen and fibroblast cells (i.e., ECM) that provides structure to host tissue. The ECM in $\Omega_{H}$ can deform in response to pressure. Following previous work, we assume constant cell density and model cellular motion within the ECM as incompressible fluid flow in a porous medium. Response of cells and ECM to pressure is governed by Darcy's law:

$$
\mathbf{u}=-\mu_{p} \nabla P, \quad x \in \Omega_{V} \cup \Omega_{H}
$$

where cellular mobility $\mu_{p}=\mu(\mathbf{x})$ measures overall ability of tissue to respond to pressure. Note that $\mu_{p}$ also measures permeability of tissue to tumor cells. See [7] and [43] for further motivation of this approach from a mixture modeling viewpoint. When tumor cells are hypoxic, cellular pathways that stimulate 
cell migration may be activated $[95,110,126,66,166]$. This may be modeled by increasing mobility $\mu_{p}$ as nutrient level decreases or as a tactic response to nutrient gradients [78]. Here, we focus upon the effects of proliferative pressure only; effects of increased cellular mobility in response to hypoxia will be considered in future work [13]. Outward normal velocity $V$ of the tumor boundary $\Sigma$ is given by

$$
V=\mathbf{u} \cdot \mathbf{n}=-\mu_{p} \nabla P \cdot \mathbf{n}
$$

where $\mathbf{n}$ is the outward unit normal vector along $\Sigma$.

\section{Proliferation, Apoptosis, and Necrosis}

In the viable region $\Omega_{V}$, proliferation increases the number of tumor cells and thus the volume occupied by the viable region. Apoptosis decreases total volume of $\Omega_{V}$ at a constant rate $\lambda_{A}$. We assume that cell birth and death are in balance in $\Omega_{H}$, and so there is no change in volume in that region. (Note that if there are no cells in $\Omega_{H}$, then there is no cell birth or death, and the assumption still holds.) In fact, poorly vascularized tumors are often hypoxic, leading to anaerobic glycolysis and acidosis [85, 86]. Non-cancerous cells struggle survive in this condition, providing a relative survival advantage for tumor cells and a potential volume loss in $\Omega_{H}$ when cells are present.

As a computational convenience, we can achieve the correct volume loss by continuously extending the velocity $\mathbf{u}$ into $\Omega_{N}$. We also assume that the normal velocity is continuous across the tumor boundary $\Sigma$, i.e., voids do not form between tumor and host tissue. Therefore, we choose our extension such that the normal velocity is continuous across the necrotic boundary, i.e., $[\mathbf{u} \cdot \mathbf{n}]=0$ across $\Sigma_{N}$. Because the nutrient level determines $\Sigma_{N}$, the latter is not a material boundary and is not advected by the velocity field $\mathbf{u}$; the extension of the velocity field is used solely to yield the correct volume change in the tumor necrotic core. One means to attain this is to extend the pressure continuously into the necrotic core as well, by setting $\bar{\varepsilon}=0$ in 22 :

$$
\begin{array}{r}
\mathbf{u}=-\mu_{p} \nabla P, \quad \mathbf{x} \in \Omega_{N} \\
{[P]=0, \quad \mathbf{x} \in \Sigma_{N}} \\
{\left[-\mu_{p} \nabla P \cdot \mathbf{n}\right]=0, \quad \mathbf{x} \in \Sigma_{N}}
\end{array}
$$

The jump condition $[P]=0$ across $\Sigma_{N}$ models low cellular adhesion and is consistent with increased cellular mobility observed in hypoxic cells [33, 44, $96,165,173,166]$. Note that (38) automatically satisfies $[\mathbf{u} \cdot \mathbf{n}]=0$ on $\Sigma_{N}$.

\section{Mechanical Pressure}

We can obtain an equation for the mechanical pressure in $\Omega_{T} \cup \Omega_{H}$ by noting the pressure extension in Eq. (38): 


$$
\nabla \cdot\left(\mu_{p} \nabla P\right)= \begin{cases}0 & \text { if } \mathbf{x} \in \Omega_{H} \\ b c-\lambda_{A} & \text { if } \mathbf{x} \in \Omega_{V} \\ -\lambda_{N} & \text { if } \mathbf{x} \in \Omega_{N}\end{cases}
$$

By the continuity of the normal velocity across the tumor boundary, by Darcy's law (36) there is no jump in the normal derivative $\mu_{p} \nabla P \cdot \mathbf{n}$ across $\Sigma$. Following [59] and others, we model cell-cell adhesion forces in the tumor by introducing a Laplace-Young surface tension boundary condition. Therefore,

$$
\begin{gathered}
{[P]=\gamma \kappa, \quad \mathbf{x} \in \Sigma} \\
0=[\mathbf{u} \cdot \mathbf{n}]=-\left[\mu_{p} \nabla P \cdot \mathbf{n}\right], \quad \mathbf{x} \in \Sigma
\end{gathered}
$$

where $\kappa$ is the mean curvature and $\gamma$ is a constant cell-cell adhesion parameter. Cellular proliferation and death are in balance outside of $\Omega_{T} \cup \Omega_{H}$. Therefore,

$$
P \equiv P_{\infty}, \quad \mathbf{x} \in \partial\left(\Omega_{T} \cup \Omega_{H}\right)
$$

on the far-field boundary.

Here we consider the special case of avascular growth in piecewise homogeneous tissue and take $\mu_{p} \equiv \mu$ in $\Omega_{H}$ and $\mu_{p} \equiv 1$ in $\Omega_{T}$, where $\mu$ is a constant. Note that because $\mu$ is constant within the tumor (and across $\Sigma_{N}$ ), the pressure boundary conditions across $\Sigma_{N}$ in (38) are automatically satisfied for any $C^{1}$ smooth solution $P$.

\subsection{Results}

We investigate the effects of the tumor microenvironment on the morphology and growth patterns of 2D, avascular tumors growing into piecewise homogeneous tissues. Recall that $D$ characterizes relative nutrient diffusivity, $A$ is amount of apoptosis, $G$ measures tumor aggressiveness (proliferation compared to cellular adhesion), $G_{N}$ characterizes rate of degradation of the necrotic tissue, and $N$ is threshold nutrient level for tumor cell viability. Viable rim size is determined by $D, A$, and $N$, while the necrotic core size is determined by $G_{N}$. In all simulations, we set the apoptosis parameter $A=0$ because the tumors are assumed to ignore inhibitory signals for self-destruction (apoptosis). We numerically compute the solutions using a computational mesh with $\triangle x=\triangle y=0.08$. All tumors are simulated to a scaled nondimensional time of $T=G t=\lambda_{M} t^{\prime}=20$, where $t^{\prime}$ is dimensional time. (The dimensional time is given by $t^{\prime}=T / \lambda_{M}$.) Because $\lambda_{M}^{-1} \sim 1$ day, this non-dimensional time allows us to compare tumors of varying simulated genotypes at fixed physical times (e.g., $T=20 \approx 20$ days).

We characterize the effects of the modeled tumor microenvironment on growth by presenting a morphology diagram (Fig. 12). We simulate growth over a wide range of microenvironmental parameters $(D$ and $\mu$ ) with $G=20$; 
$G_{N}=1$, and $N=0.35$, each with identical initial shape. Later, we shall consider the effect of $G, G_{N}$, and $N$. We let $D \in\{1,50,100, \infty\}$ and $\mu \in$ $\{0.25,1,50, \infty\}$. When $D=\infty$, we set $\sigma \equiv 1$ in non-encapsulated regions of $\Omega_{H}$ and only solve the Poisson equation for $\sigma$ in $\Omega_{T}$ and the encapsulated portions of $\Omega_{H}$ (with diffusion constant 1). Likewise, when $\mu=\infty$, we set $p \equiv 0$ in non-encapsulated regions of $\Omega_{H}$ and only solve the Poisson equation for $p$ in $\Omega_{T}$ and the encapsulated portions of $\Omega_{H}$ (with mobility 1). In Fig. 12 , we plot the shape of each tumor at time $T=20.0$. In all figures, the black regions denote $\Omega_{N}$ where the tumor is necrotic, the gray regions show the viable tumor region $\Omega_{V}$, and the white regions correspond to $\Omega_{H}$, which consists of the ECM, non-cancerous cells, and any other material outside of the tumor.

On the horizontal axis, we vary the nutrient diffusivity of the surrounding tissue; as $D$ increases from left to right, the simulated microenvironment varies from nutrient poor to nutrient rich. On the vertical axis, we vary the mobility of the surrounding material; as $\mu$ increases from bottom to top, the microenvironment ranges from low mobility to high mobility. The greater the mobility $\mu$, the greater the ability of the external, non-cancerous tissue to respond to the pressure generated by the growing tumor, and tumor cells are more able to penetrate the tissue. We observe three distinct tumor morphologies through this broad range of simulated tissue types. In the nutrient poor regime on the left side of the diagram, tumors demonstrate fragmenting growth, characterized by the repeated breakup of the tumor in response to the low nutrient level. The nutrient-rich, low-mobility regime in the bottom right of the morphology diagram is characterized by fingering growth, where buds develop on the tumor boundary that invade the surrounding tissue, forming long, invasive fingers. The nutrient-rich, high-mobility regime in the top right of the diagram demonstrates compact/hollow growth, where the tumors tend to grow into spheroids and typically form abscesses filled with non-cancerous tissue and fluid, similar to a necrotic core. These morphologies are similar to those observed experimentally in vitro (e.g., [74].

We have found that the tumor morphologies in Fig. 12 are qualitatively similar when recomputed with different genetic characteristics (modeled by $A$, $G, G_{N}$, and $N$ ), although, as demonstrated in Sec. 3.3, large changes in the genetic parameter values can shift the morphology from one type to another. Therefore, a tumor's morphology depends primarily upon the characteristics of the microenvironment, as we shall see next.

\section{Fragmenting Growth Into Nutrient-Poor Microenvironments}

Tumors growing into nutrient-poor microenvironments demonstrate repeated fragmentation through a wide range of mitosis rates (governed by the parameter $G$ ) and necrotic tissue degradation rates $\left(G_{N}\right)$. Tumor fragmentation is observed in almost all cases, particularly for fast-proliferating, aggressive tumors with higher values of $G$. An increased aggressiveness $(G)$ increases the 
rate of tumor fragmentation. Similarly, increasing the rate of necrotic tissue degradation $\left(G_{N}\right)$ tends to destabilize the tumor, also leading to an increased rate of fragmentation. However, this effect is highly nonlinear: if $G_{N}$ is large relative to $G$, then proliferation, necrosis, and cellular adhesion can balance to maintain spheroids and prevent further tumor fragmentation. Note that for sufficiently low levels of tumor aggressiveness (e.g., $G=0.10$ ), tumor instability decreases until the steady-state configuration is as tumor spheroids, as predicted in [59] for non-necrotic tumors.

We found that the volume fractions of viable and necrotic tissue were largely independent of the tumor aggressiveness parameter $G$ and the microenvironmental characteristics $(D$ and $\mu$ ) and were primarily functions of $N$ and $G_{N}$. The occurrence of repeated tumor fragmentation is found to occur over a broad range of $G$ and $G_{N}$. This demonstrates that in the nutrient-poor regime tumor morphology is largely determined by the characteristics of the surrounding microenvironment, while the genetic characteristics of the tumor $\left(G, G_{N}, A\right.$, and $\left.N\right)$ determine the size and rate of evolution of the tumor. In addition, increasing the apoptosis rate $A$ to positive values results in similar morphological behavior, only with more rapid tumor fragmentation and a greater number of fragments (results not shown).

The finding that tumor morphology in the nutrient-poor regime depends primarily upon the tumor microenvironment and not upon the tumor's genetic characteristics has important implications for cancer treatment. In antiangiogenic therapy, drugs are supplied to prevent the neovascularization of the growing tumor and the surrounding tissue. The resulting nutrient-poor microenvironment may cause the tumors to fragment and invade nearby tissues, particularly in higher-mobility tissues. This can negate the positive effects of anti-angiogenic therapy and lead to recurrence and metastasis. This result is consistent with the findings of [55], who suggested that combining anti-angiogenic therapy with adhesion therapy may counteract the negative problems associated with tumor fragmentation in the nutrient-poor regime.

\section{Invasive, Fingering Growth}

In Fig. 13, we show the evolution of a tumor growing into a low-mobility, nutrient-rich tissue, where $D=50$ and $\mu=1$. As in the previous section, $G=20, G_{N}=1$, and $N=0.35$. Because nutrient readily diffuses through the surrounding tissue $\Omega_{H}$, the tumor is initially non-necrotic, allowing for unchecked growth and the development of buds on the tumor periphery that protrude into the surrounding tissue (see time $T=10$ in Fig. 13). Due to the cell-cell adhesion (modeled by the pressure jump in (40), the proliferationinduced mechanical pressure is greatest surrounding any protrusions of the tumor into the healthy tissue and approximately zero near flatter regions of the tumor boundary. Because the cellular mobility $\mu$ is low in the noncancerous tissue, the individual cells and the ECM cannot move to equilibrate the pressure. As a result, the cellular velocity field is mostly parallel to the 
buds, in spite of adequate nutrient levels between the growing buds. This makes it difficult for buds to merge, leading to the formation of long, invasive fingers (see $T=30.0-50.0$ in Fig. 13). The net effect is highly invasive growth into the surrounding tissue.

Within the nutrient-rich, low-mobility tissue regime, we examined two levels of tissue mobility $(\mu \in\{0.25,1\}$ ) and three nutrient diffusivities $(D \in\{50,100, \infty\})$, for a total of six combination of mobility and nutrient diffusivity. We found that the shape depended primarily upon the tissue mobility: the three lower-mobility tissue examples $(\mu=0.25)$ had an overall higher shape parameter than the higher-mobility tissue $(\mu=1)$, which reflects a higher degree of deformation. This trend is indeed observed in the morphologies along the $\mu=0.25$ row of Fig. 12. This is because the lower the tissue mobility, the more difficult it is for cells in the healthy tissue to overcome the cell-cell and cell-ECM adhesion and move to equilibrate pressure variations, and the more difficult it is for the ECM to deform in response to the pressure, allowing for the formation of sharper corners and greater shape instabilities.

Overall, the larger deformation in the lower-mobility tissue simulations leads to overall larger perimeters in the low-mobility tissue cases than in the higher-mobility tissue cases. As a result of the increased surface area, the low-mobility tissue tumors had greater access to nutrient. This leads to a surprising result: the increased morphological instability from growing into lower-mobility tissues improves access to nutrient and leads to larger tumors, for each fixed nutrient diffusivity, the volume of the viable area of each tumor was larger for the lower-mobility tissue simulation $(\mu=0.25)$ than for the corresponding higher-mobility tissue example $(\mu=1)$. For all examples, interface length, relative to a circle with same area, steadily rose as a function of time, which reflects the increasing shape instability as the tumors invade the surrounding tissue; this is characteristic of invasive, fingering growth. This has implications for therapies that target cell-cell and cell-ECM adhesiveness: if the therapy decreases the mobility in the surrounding microenvironment (by increasing the cell-cell or cell-ECM adhesiveness or rendering the ECM more rigid), then invasive, fingering growth into the surrounding tissue is likely. Likewise, a treatment that decreases the permeability of the host tissue to tumor cells may lead to an increase in tumor invasiveness.

The finger width is most strongly dependent upon the nutrient diffusivity $D$, and largely independent of the tissue mobility $\mu$. As the nutrient diffusivity increases, nutrient is better able to diffuse between the growing fingers, allowing the nutrient to penetrate farther into the fingers. This allows the tumor to support thicker fingers. In all cases, the length scale tended toward a roughly fixed value, which demonstrates that each tissue can support a specific finger thickness.

In Fig. 14, we examine the effect of the tumor aggressiveness parameter $G$ and the necrotic degradation parameter $G_{N}$ on the invasive, fingering morphology. We fix $D=50, \mu=1 ; N=0.35$, and take $0.1 \leq G_{N} \leq 10.0$ and $1 \leq G \leq 100$. For lower tumor aggressiveness values $(G=1)$ and $G_{N} \geq 1$, the 
fingering effect was significantly reduced, resulting in more stable, tubularshaped tumors, an effect that has been observed in experiments [74]. These structures form because tumor cell proliferation (the numerator of $G$ ) and cell-cell adhesion (the denominator of $G$ ) are roughly in balance when $G=1$. The competition between proliferationand adhesion smoothes but does not completely prevent shape instabilities, which may continue to grow. For sufficiently large values of $G$, the invasive fingering morphology was observed in all simulated tumors. For lower values of $G_{N}$ (left column in Fig. 14), the low rate of degradation of the necrotic tumor tissue leads to the formation of very wide fingers; this morphology may be better described as a collection of spheroids. As $G_{N}$ is increased, the necrotic core is degraded more quickly, leading to a decreased finger thickness, less stable morphology, and more aggressive tissue invasion. As $G_{N}$ is increased toward $G_{N}=10$ (right column in Fig. 14), the finger thickness is decreased to the point where the tumor periodically breaks into fragments and then reconnects, leading to the encapsulation of non-cancerous tissue (white enclosed regions). This morphology, which we refer to as compact/hollow, is characterized by the presence of a large abscess containing a mixture of necrotic cells, fluid, ECM, and cellular debris, much like a necrotic core. In a long-time simulation of a tumor with the compact/hollow morphology, the effect of $G_{N}$ on growth is seen to be nonmonotonic: increasing $G_{N}$ at first limits the size of the tumor by decreasing the thickness of the invasive fingers and limiting the overall spread of the tumor, but after a certain point, instability breaks the tumor and allows greater spread through the surrounding non-cancerous tissue.

\section{Compact, Hollow Growth}

We examine the effect of the tumor aggressiveness $G$ and the necrotic degradation rate $G_{N}$ on the compact tumor morphology. In these simulations, we fix $D=50 ; \mu=\infty$, and $N=0.35$. For lower values of $G$, the tumors remain in compact morphologies that fail to encapsulate non-cancerous tissue, although shape instabilities may occur at long times. When $G=1$, cell proliferation (numerator of $G$ ) and adhesion (denominator of $G$ ) are roughly in balance, which shrinks but does not completely prevent shape instabilities. For larger values of $G$, the cell proliferation rate outstrips cell-cell adhesion, resulting in folds in the outer tumor surface that encapsulate non-cancerous tissue. For fixed values of $G$, we find that increasing the necrotic tissue degradation rate parameter $G_{N}$ shrinks the necrotic volume fraction of the tumors. In the cases where non-cancerous tissue has been encapsulated $(G>1)$, increasing $G_{N}$ increases the size of the central tumor abscess. Lastly, as in the fragmenting and fingering cases, we find that varying $N$ changes the tumor evolution quantitatively but not qualitatively. As $N$ increases, the thickness of the viable rim decreases, the necrotic volume fraction increases, and morphological instability also increases. 


\section{Complex Tissue}

We model growth in a complex, heterogeneous brain tissue as shown in the first frame in Fig. 15. We define $B$ as the pre-existing blood vessel density (as an indicator of bulk supply of nutrient to the vascularized tumor tissue). In the white region at the right side, $\mu=0.0001, D=0.0001$, and $B=0$, which models a rigid material such as the skull. In the black regions, $\mu=10, D=1$, and $B=0$, which models an incompressible fluid (cerebrospinal fluid). The light and dark gray regions model tissues of differing biomechanical properties (white and gray matter). In the light gray regions, $\mu=1.5, D=1$, and $B=1$; in the dark gray regions, $\mu=0.5, D=1$, and $B=1$. The tumor is denoted by a white thin boundary in the middle right of the frame. We smoothed $\mu$, $B$, and $D$ using a Gaussian filter with standard deviation $\sigma=3 \triangle x=0.3$ to satisfy smoothness requirements of the reaction-diffusion equations. We used (linear) extrapolation boundary conditions on the pressure along $x=0, y=0$, and $y=50$ to simulate growth into a larger (not shown) tissue and set $p=0$ along the rigid boundary at $x=50$.

We simulated from $t=0$ to $t=60$. Using a $3.3 \mathrm{GHz}$ Pentium 4 workstation and a $\mathrm{C}++$ implementation, the $501 \times 501$ simulation required less than 24 hours to compute. Because the (mitosis) time scale ranges from approximately 18 to 36 hours for this problem, this corresponds to 45 to 90 days of growth. We plot the solution in $t=10.0$ (approximately 10 days) increments in Fig. 15. In these plots, regions corresponding to viable tumor tissue (where $\left.c>c_{H}\right)$, hypoxic tumor tissue $\left(c_{H} \geq c>c_{N}\right)$, and necrotic tumor cells $\left(c_{N} \geq c\right)$ are shown. In hypoxic tissue, there is assumed to be no proliferation. In this simulation, the tumor grows rapidly until the nutrient level drops below $c_{H}=0.30$, at which time a large portion of the tumor becomes hypoxic. The tumor continues to grow at a slower rate until the interior of the tumor becomes necrotic (see $t=10.0$ ). This causes non-uniform volume loss within the tumor and contributes to morphological instability. Note that because the biomechanical responsiveness is continuous across the tumor boundary and the microenvironment has a moderate nutrient gradient, this simulation corresponds to the border between the invasive, fingering growth regime and the invasive, fragmenting growth regime that was investigated earlier.

However, additional effects can be seen that were not observed before. As the tumor grows out of the biomechanically permissive tissue (light gray; $\mu=1.5$ ) and into the biomechanically resistant tissue (dark gray; $\mu=0.5$ ), its rate of invasion into the tissue slows (see $t=20.0$ ). This results in preferential growth into the permissive (light gray) material, a trend which can be clearly seen from $t=30.0$ onward. When the tumor grows through the resistive tissue (dark gray) and reaches the fluid (black) $(t=40.0)$, the tumor experiences a sudden drop in biomechanical resistance to growth. As a result, the tumor grows rapidly and preferentially in the $1 / 2 \mathrm{~mm}$ fluid structures that separate the tissue $(t=50.0-60.0)$. Such growth patterns are not observed when simulating homogeneous tissues. Other observed differences are due to our 
new treatment of hypoxic (quiescent) tumor cells. Certain regions that had previously been classified as necrotic (in $[132,133,134,135])$ are now treated as quiescent. As a result, tumor volume loss is reduced, and in particular, this may result in large hypoxic regions that have little or no viable rim. Had these regions been treated as necrotic, the invasive fingers would have been thinner, and the tumor may have fragmented. Therefore, the separate treatment of the hypoxic regions can have a significant impact on the details of the invasive morphology of the tumor.

\subsection{Summary}

In this work $[135,136]$ we developed a framework to investigate the interaction between avascular solid tumors and their microenvironment. In particular, we model perfusion of nutrient through a tumor and surrounding microenvironment, build-up of pressure in the tissue from proliferation of cancerous cells, cell-cell and cell-ECM adhesion, and loss of tumor volume due to necrosis. We observed three distinct morphologies: fragmenting, invasive/fingering, and compact/hollow growth. If the microenvironment is nutrient poor, tumors tend to break into small fragments and spread throughout the microenvironment, regardless of cellular mobility. Within this nutrient-poor growth regime, decreasing microenvironmental mobility (by increasing noncancerous cell-cell and cell-ECM adhesion or increasing ECM rigidity) decreases extent of fragmentation and slows invasion into surrounding tissue, but does not completely prevent hypoxia-induced morphological instability. (Unstable tumor morphologies in the nutrient-poor regime have been observed by [10] and [55].) An invasive, fingering morphology was found in cases of growth into nutrient-rich, low-mobility microenvironments. Increasing nutrient perfusion does not prevent this invasive morphology, and the lower the microenvironmental mobility, the greater the degree of morphological instability and invasiveness. Tumors growing into nutrient-rich, high-mobility tissues develop compact/ hollow morphologies. A hallmark of this growth regime is formation and merger of buds on the tumor periphery, which leads to encapsulation of noncancerous regions and formation of a large abscess that qualitatively is similar to a necrotic core.

Since decreasing nutrient levels in the microenvironment tends to increase tumor fragmentation and invasion into the surrounding tissue, this may have to be taken into consideration during anti-angiogenic therapies, as it could lead to morphological instability in the form of fragmentation and invasion. A number of experimental studies have recently shown that anti-angiogenic therapies may have this result $[180,177,61,173,185,122,24]$. Conversely, we found that increasing nutrient levels leads to greater morphological stability and increased tumor compactness, thereby rendering some tumors more resectable. These results support the contention [55] that treatments that seek to normalize tumor vasculature (by selectively "pruning" weak blood vessels with targeted anti-angiogenic therapy) may stabilize tumor morphology 
by providing increased access to nutrient. Since such treatments may also increase the accessibility to chemotherapeutic agents [105, 188], our results provide additional support for the use of targeted anti-angiogenic therapy as adjuvant to chemotherapy and resection.

As pointed out by [55], another approach to therapy is to use anti-invasive drugs such as Met inhibitors [30, 20, 146] or hepatocyte growth factor (HGF) antagonists $[60,144]$ in addition to anti-angiogenic therapies. Such therapies affect cell-cell and cell-ECM adhesive properties of the tumor. A recent experimental study on mouse models of malignant glioma shows that fragmentation can be prevented, and tumor satellites may be eliminated by a combined antiangiogenic and anti-invasive therapy [24]. In the nutrient-poor growth regime, increasing cell-cell and cell-ECM adhesion of the microenvironment can help limit the rate of fragmentation and the extent of invasion. Decreasing permeability of the microenvironmental ECM to tumor cells by other means, such as making the ECM more dense, stiffer, and less able to support tumor cell movement, could also attain this effect.

Interestingly, the opposite approach is warranted in the nutrient-rich growth regime. In this case, decreasing cell-cell and cell-ECM adhesion in the microenvironment (while leaving tumor cells unaffected) or increasing the permeability of the microenvironment to tumor cells decreases invasive fingering. This could also be accomplished by increasing tumor cell-cell and cell-ECM adhesion or by decreasing the stiffness or density of the surrounding ECM, respectively. Such subtleties highlight the importance of considering tumormicroenvironment interactions when planning therapies that affect adhesive and mechanical properties of a tumor, its surrounding tissue, or both.

\section{Vascularized 3-D Tumor Growth Using a Diffuse Interface Approach"}

\subsection{Overview}

This section describes development of a model of Functional Collective CellMigration Units (FCCMU) [72] that describes the large-scale morphology and 3 -D cell spatial arrangements during tumor growth and invasion. This includes the application of mathematical and empirical methods to quantify the competition between cell substrate gradient-related pro-invasion phenomena and molecular forces that govern proliferation and taxis, and forces opposing invasion through cell adhesion. The latter, under normoxic conditions, often enforce compact non-infiltrative tumor morphology while local oxygen gradients promote invasion $[190,156,185,120,160,122,24,177,173,174,61,55$, $74,135]$. Interactions between cellular proliferation and adhesion and other

\footnotetext{
"| This article was published in NeuroImage, Vol. 37, Frieboes et al., Computer simulation of glioma growth and morphology, pp. S59-S70, Copyright 2007 C) Elsevier.
} 
phenotypic properties may be reflected in both the surface characteristics, e.g., stability, of the tumor-host interface and the growth characteristics of tumors $[59,55,74,87,135]$. These characteristics give rise to various tumor morphologies and influence treatment outcomes. The model thus enables the deterministic linking of collective tumor cell motion on the balance between cellular properties and the microenvironment.

We assemble this 3-D multiscale computational model of cancer as a key step towards the transition from qualitative, empirical correlations of molecular biology, histopathology, and imaging to quantitative and predictive mathematical laws founded on the underlying biology. The model provides resolution at various tissue physical scales, including the microvasculature, and quantifies functional links of molecular factors to phenotype that currently for the most part can only be tentatively established through laboratory or clinical observation. This mathematical and computational approach allows observable properties of a tumor, e.g., its morphology, to be used to both understand the underlying cellular physiology and predict subsequent growth (or treatment outcome), providing a bridge between observable, morphologic properties of the tumor and its prognosis [56, 182].

\subsection{Method Description}

The FCCMU model is based on conservation laws (e.g., of mass and momentum) with conserved variables that describe the known determinants of glioma (e.g., cell density) and with parameters that characterize a specific glioma tissue. The conservation laws consist of well established, biologically founded convection-reaction-diffusion equations that govern the densities of the tumor cell species, the diffusion of cytokines and the concentration of vital nutrients. The model describes the cells' (collective) migratory response and interaction with the extra-cellular matrix (ECM) and an evolving neovasculature. The collective tumor cell velocity depends on proliferation-driven mechanical pressure in the tissue, chemotaxis and haptotaxis due to gradients of soluble cytokines and insoluble matrix macromolecules. The cell species velocity is obtained from a Darcy's law coarse scale reformulation of the inertialess momentum equation, which is the instantaneous equilibrium among the following forces: pressure, resistance to motion (cell-adhesion), elastic forces, forces exchanged with the ECM leading to haptotaxis and chemotaxis and other mechanical effects (e.g. [59, 212, 55, 74, 181, 127, 11, 10, 12, 51, 3, 25, $48,79,16,133,90,39,40,5,135,6,43,50]$. Cells produce proteases, which degrade the matrix locally, making room for cells to migrate. In the model, matrix degradation releases cytokines and growth promoters, thus having biological effects on tumor cells (e.g., [48]). The model can account for cell-cell interactions (cell-cell adhesion and communications), high polarity, and strong pulling forces exchanged by cells and ECM [78].

The FCCMU model is coupled nonlinearly to a hybrid continuum-discrete, lattice-free model of tumor-induced angiogenesis [161, 162]. The angiogene- 
sis component describes proliferation and migration due to chemotaxis and haptotaxis of endothelial cells in response to tumor angiogenic factors (e.g. VEGF) and matrix macromolecules, respectively. The angiogenic factors are released by peri-necrotic tumor cells and host cells near the tumor-host interface $[193,186]$, which stimulate vascular endothelial cells of the brain vasculature to proliferate and begin to form vessels [107]. Anastomosed vessels may provide a source of nutrient in the tissue and may undergo spontaneous shutdown and regression during tumor growth [99]. We note that there are other related lattice-based models of tumor neo-vascularization (e.g. [191, 140]). Although tumor angiogenesis may occur via the formation of sprouts or intussuception [158], for simplicity here only the former process is incorporated in the model. Input parameter values to the model, e.g., cell proliferation and apoptosis, are estimated from in vitro cell lines and ex-vivo patient data. The parameters governing the extent of neovascularization and nutrient supply due to blood flow are estimated in part from Dynamic Contrast Enhanced Magnetic Resonance Imaging (DCE-MRI) observations in patients [152].

The model describes nutrient/oxygen delivery from the neo-vasculature (via convection and diffusion $[46,105,106]$ ) and cellular uptake, and nutrient, oxygen, and growth factor diffusion through the tumor tissue [36]. Oxygen/nutrient availability limits the fraction of cycling cells. Regions of tissue become hypoxic and then necrotic where nutrient/oxygen concentration falls below a threshold. The model describes evolution of local mass fractions of viable tumor species, necrotic and host tissues. Cell mass exchange occurs due to mutations, mitosis, necrosis, and apoptosis. Lysis rates describe the disintegration of tumor cell mass and the radial effusion of fluid away from the necrotic regions. All rates are inverse times (unit time $=1$ day). At any given time during tumor growth, the model outputs the computed values of all relevant variables at every location within the three-dimensional tumor tissue, e.g., the spatial distributions of oxygen, nutrients and tumor cell species. The result is a description of the complex, multi-scalar dynamics of in vivo 3-dimensional tumors through avascular, neo-vascular, vascular growth, and invasion stages.

\subsection{Multiscale Model}

The minimal formulation of the FCCMU model is based on reaction-diffusion equations that govern a tumor cell density, an evolving neovasculature, a vital nutrient concentration, the ECM, and matrix degrading enzymes. Extensions to include more complex biophysics, e.g., multiple nutrients, growth inhibitors and matrix remodeling, are straightforward.

In this approach, each constituent moves with its own velocity field; mass, momentum and energy equations are posed for each constituent. Through experimental comparisons and the inclusion of molecular-scale effects, we formulate functional relationships that close the FCCMU model. Generically, the reaction-diffusion equations take the form 


$$
\nu_{t}=-\nabla \cdot \mathbf{J}+\Gamma_{+}-\Gamma_{-}
$$

where $\nu$ is the evolving variable, $\mathbf{J}$ is the flux, $\Gamma_{+}$and $\Gamma_{-}$are the sources and sinks. Letting $\nu=\rho_{i}, \sigma, f, m$, respectively be the tumor cell density of species $i$ or host density, the vital cell substrate concentration (e.g. oxygen), the (nondiffusible) matrix macromolecule (e.g. fibronectin [11, 10], and matrix degrading enzyme, MDE (e.g. matrix metalloproteinases, urokinase plasminogen activators $[12,10,51]$ concentrations, we may take

$$
\begin{gathered}
\mathbf{J}= \begin{cases}\rho_{i} \mathbf{u}_{i}+\mathbf{J}_{\text {mechanics }, i} & \text { if } \nu=\rho_{i} \\
\sigma \mathbf{u}_{W}-D_{\sigma} \nabla \sigma & \text { if } \nu=\sigma \\
0 & \text { if } \nu=f\end{cases} \\
\Gamma_{+}= \begin{cases}\rho_{i} \lambda_{\text {prolif }, i}+\sum_{j} S_{i j}^{+} & \text {if } \nu=\rho_{i} \\
\lambda_{\text {blood }}-D_{m} \nabla m & \text { if } \nu=\sigma \\
\lambda_{f} & \text { if } \nu=f \\
\lambda_{\text {mde }} & \text { if } \nu=m\end{cases} \\
\Gamma_{-}= \begin{cases}\rho_{i} \lambda_{\text {death }, i}+\sum_{j} S_{i j}^{-} & \text {if } \nu=\rho_{i} \\
\lambda_{\sigma, \text { uptake }} & \text { if } \nu=\sigma \\
\lambda_{f, \text { degrade }} & \text { if } \nu=f \\
\lambda_{\text {mde,degrade }} & \text { if } y\end{cases}
\end{gathered}
$$

where $\mathbf{u}_{i}$ is the cell-velocity of species or host $i, \mathbf{u}_{w}$ is the velocity of water (i.e. assuming transport of chemical factors is primarily through the interstitial liquid), the $D$ 's are diffusion constants, and $\lambda_{\text {prolif }}, \lambda_{\text {death }}, \lambda_{\text {blood }}, \lambda_{\text {uptake }}$, $\lambda_{f}, \lambda_{f, \text { degrade }}, \lambda_{\text {mde }}$, and $\lambda_{\text {mde,degrade }}$ are the mitosis, apoptosis and necrosis, blood-tissue nutrient transfer and uptake and decay rates, respectively, for matrix molecules and MDE. An additional equation (not shown) is posed for the mass fraction of water. The flux $\mathbf{J}_{\text {mechanics }, i}$ accounts for the mechanical interactions among the different cell species. A major component of the FCCMU model is the development of the constitutive law for $\mathbf{J}_{\text {mechanics, } i}$. This is obtained from a variational approach from an energy formulation that accounts for the mechanical forces, e.g. cell-cell and cell-matrix adhesion, and elastic effects (residual stress). A feature of this approach is the incorporation 
of a novel continuum model of adhesion in this flux. Following the variational approach developed for diffuse interface models of multiphase flows and materials by Lowengrub and coworkers (e.g., [130, 124, 123, 112, 113, 203, 114]) and others (e.g. $[84,101,11])$ we introduce a continuum model of cell-cell and cellmatrix adhesion energy that can be written as an integral taken over the entire tumor/host domain $E_{\text {adhesion } i}=\int f_{i}\left(\rho_{1}, \ldots, \rho_{N}\right)+\frac{1}{2} \sum_{j=1}^{N-1} \varepsilon_{i, j}^{2}\left|\nabla \rho_{j}\right|^{2} d x$. The first term is a bulk energy, which accounts for the degree of miscibility of cell and host species, as directed by experiments. The second term introduces cell-cell adhesion forces that generate a surface tension between the phases and further accounts for intermixing across a diffuse interface of thickness that roughly scales with $\varepsilon_{i, i}$. Typically, the cell-adhesion energy enforces phase separation of tumor and host tissues sharing a diffuse interface with thickness 1-100 $\mu \mathrm{m}$. Under the assumption that tumor cells prefer to stay bonded with each other rather than being in any other configuration, that the cell density is roughly constant and that there is only isotropic stress (pressure $p$ ), this reduces, in an asymptotic limit, to the "jump" boundary condition $[p]=\tau \kappa$ where $\tau$ measures the affinity and $\kappa$ is the total curvature of the interface $[202,73]$. This is akin to surface tension in multiphase flows and can also be used to describe tumor encapsulation by ECM fragments and is characteristic of collective cell migration [78].

A thermodynamically consistent constitutive law for the flux $\mathbf{J}_{\text {mechanics }, i}$ is obtained by taking the gradient of the variational derivative of the total energy: $\mathbf{J}_{\text {mechanics }, i} \infty \nabla\left(\delta E_{\text {mechanics }, i} / \delta \rho_{i}\right)$, where $E_{\text {mechanics }, i}$ is obtained by adding the contributions from each mechanism modeled, i.e. adhesion, elasticity, etc. The velocities $\mathbf{u}_{i}$ and $\mathbf{u}_{w}$ are determined from momentum equations. For example, following previous approaches (e.g., [59, 212, 55, $74,127,133,135])$ that are reformulations and generalizations of models in $[90,39,40,3,25,48]$ and neglecting viscoelastic effects, we take Darcy's law as a coarse scale reformulation of the inertialess momentum equation, which is the instantaneous equilibrium among the following forces: pressure, resistance to motion, elastic forces, forces exchanged with the ECM leading to haptoand chemo- taxis and other mechanical effects within $E_{\text {mechanics }}$ as discussed above. This leads to

$$
\mathbf{u}_{i}=-M_{i} \nabla p+\gamma_{i}\left(\delta E_{\text {mechanics }, i} / \delta \rho_{i}\right) \nabla \rho_{i}+\chi_{f, i} \nabla f+\chi_{\sigma, i} \nabla \sigma
$$

where $p$ is the pressure (isotropic stress) and $M, \gamma_{i}, \chi_{f}$, and $\chi_{\sigma}$ are the spatially inhomogeneous mobility, mechano-, hapto- haptotaxis and chemo- taxis tensors that also take into account cell-matrix adhesion. The parameter $M$ depends on the extent of cell-to-cell and cell-to-ECM adhesion in bulk regions. Since a number of different parameters in the model describe various effects of cell-cell and cell-ECM adhesion it is expected that this model should have enough complexity to reproduce nontrivial and non-monotonic dependences of migration on CAMs [78]. Note that other models such as Stokes, viscoelastic and nonlinear-elastic/plastic can be incorporated as required. 
The FCCMU continuum-scale convection-reaction-diffusion equations are solved numerically using a novel adaptive finite-difference method [202, 73, 201]. This method features an adaptive, block-structured Cartesian mesh refinement algorithm (e.g. [27]), centered differences in space and an implicit time discretization for which there is no stability constraint on the space and time steps. The nonlinear equations at the implicit time level are solved efficiently using a multilevel, nonlinear multigrid method (e.g. [31]).

\subsection{Tumor Vasculature}

We model the physiology and evolution of glioma neovasculature in 3D using a hybrid continuum-discrete, lattice-free model of tumor angiogenesiswhich is a refinement of earlier work $[161,162]$. This was shown to create dendritic structures consistent with experimentally observed tumor capillaries [125, 189]. This random walk model generates vascular topology based on tumor angiogenic factors, e.g., vascular endothelial growth factor (VEGF) [193], represented by a single continuum variable that reflects the excess of proangiogenic factors compared to inhibitory ones. Peri-necrotic tumor cells and host tissue cells close to the tumor boundary are assumed to be a source of angiogenic factors (e.g., VEGF). Endothelial cells near the sprout tips proliferate and their migration is described by chemotaxis and haptotaxis (e.g., motion up gradients of angiogenic factors and matrix proteins such as fibronectin). For simplicity, only leading endothelial cells are modelled and trailing cells passively follow. The vasculature architecture, i.e., interconnectedness and anastomoses, is captured via a set of rules, e.g., a leading endothelial cell has a fixed probability of branching at each time step while anastomosis occurs if a leading endothelial cell crosses a vessel trailing path. Glioma vessels are more tortuous than normal vessels [34]. This can be quantified by various means including a "Sum of Angles Metric" (SOAM) that sums total curvature along a space curve and normalizes by path length, indicating high frequency, low-amplitude sine waves or coils [34].

The tumor-induced vasculature does not initially conduct blood, as the vessels need to form loops first (anastomosis) [19]. As observed experimentally, the neovasculature model may also account for increasing vessel diameters and spontaneous shutdown and consecutive regression of initially functioning tumor vessel segments or whole microvascular areas [99]. Here, functional anastomosed vessels were assumed to provide a source of nutrient in the tissue proportionally to local pressure.

\subsection{Calculation of Model Parameters}

Previous measurements of growth and histopathology of in vitro ACBT (human glioblastoma multiforme) tumor spheroids [74], and of human glioma [23] were used to inform the parameters of the simulation presented herein. Briefly, higher-grade glioma mitosis and apoptosis rates were taken to be 1 
day $^{-1}$ and 0.32 day $^{-1}$ respectively. The characteristic time scale was taken to be the inverse mitosis rate. The diffusion penetration length was measured to be $100 \mu \mathrm{m}[59,74]$, and is used herein as the characteristic unit of length. The necrosis threshold was taken to be $\sigma_{N} / \sigma_{V}=0.5$, where $\sigma_{N}$ is the nutrient concentration needed for viability and $\sigma_{V}$ is the nutrient concentration in the far-field. Mutation rates from low to high grade glioma were also estimated in previous work [23] but not utilized here. In previous work, a critical value of cell adhesion parameter was determined from shape stability analysis of experimental and simulated spheroids [74]: compact spherical morphologies exist only for sufficiently large adhesion, which is implemented via the parameter (after non-dimensionalization) in (47). The above set of parameters provided the baseline for our simulations (the results show a simulation using a sub-critical value of the adhesion parameter). Simulations were performed using one fixed set of parameters as described above. Parameter sensitivity studies were performed where cell adhesion $\left(\gamma_{i}\right)$ and cell chemotaxis $\left(\chi_{\sigma, i}\right)$ parameters were varied to study their effect on the morphology of infiltrating collective-cell patterns (i.e., cell chains vs. strands vs. detached clusters [78]). Representative resulting morphologies are reported elsewhere and confirm that for relatively low cell adhesion morphologic instability occurs when nutrient heterogeneity is present leading to the development of infiltrative cell protrusions [59, 74, 135, 23]. The shape features of these protrusions further depends on the relative strengths of cell proliferation and cell chemotaxis. A control was provided by simulations corresponding to relatively high cell adhesion, for which tumors grow spherical and morphologic instability does not occur.

\subsection{Results}

Figure 16 shows a mm-sized glioblastoma during early stages of growth simulated using our 3-D multiscale model. The model predicts regions of viable cells, necrosis in inner tumor areas, and a tortuous neovasculature as observed in vivo [34]. Conducting vessels are capable of releasing nutrient. The rate of nutrient released may depend on their age and the solid pressure in the tissue. The vessels migrate towards the tumor/host interface since peri-necrotic tumor cells and host tissue cells close to the tumor boundary are assumed to produce angiogenic factors and other regulators. The tumor eventually coopts and engulfs the vessels. The tumor-induced vasculature does not initially conduct blood, as the vessels need to form loops first (anastomosis) [19], i.e., more mature vessels that have anastomosed conduct blood and may release nutrient. By hypothesizing the underlying mechanisms driving these phenomena, the model enables a quantitative analysis, e.g., viable region thickness of about 100-200 $\mu \mathrm{m}$ and extent of necrosis as seen in Fig. 16 are shown to be strongly dependent on diffusion gradients of oxygen/nutrient in the microenvironment and agree with previous experiments [94, 74]. Chaotic angiogenesis leads to heterogeneous perfusion in the tumor that then might be responsi- 
ble for regression of parts of the vascular network and necrosis of tumor cells $[45,158]$, further enhancing variable tumor cell proliferation. By taking vessel maturation into account, the simulations correctly predict that as tumor size increases, inner vessels may regress or shut down, leading to nutrient depletion and resulting in the formation of a large necrotic core (data not shown), as observed in patients. For example, cm-sized human glioblastoma at later stages as seen through Magnetic Resonance Imaging (MRI) in patients (e.g., [205]) is composed of viable cells delineating its boundary and surrounding extensive necrosis in its inner region.

The multiscale model enables the prediction of tumor morphology by quantifying the spatial diffusion gradients of cell substrates maintained by heterogeneous cell proliferation and an abnormal, constantly evolving vasculature. Figure 16 shows a simulated time-sequence over the course of three months predicting that the glioblastoma grows with a thin layer of viable tissue on its periphery, displacing nearby tissue and internally generating necrosis. The morphology is directly influenced by angiogenesis, vasculature maturation, and vessel cooption $[197,19,98]$. The model predicts that the tumor boundary moves at a rate of about $50-100 \mu \mathrm{m}$ per week, presenting a mass of diameter of about $5 \mathrm{~cm}$ in one year (data not shown). These results are supported by well-known clinical observations (e.g., [148]). As the tumor grows and engulfs vessels in its vicinity, the tumor may compress the vessels [155] and disrupt flow of nutrients, leading to further necrosis and even temporary mass and vascular regression [211, 99]. A growing tumor contends with increasing mechanical resistance from normal brain tissue, which has physical properties resembling a gel $[153,68]$. Nevertheless, this resistance is insufficient to contain tumor growth, e.g., gliomas have been observed to displace cartilage [119]. Only hard bone (e.g., the skull) will be a physical barrier. The effect of such physical barriers on tumor morphology and growth can be incorporated in the multiscale model model (see methods in [72]).

\subsection{Summary}

We performed 3-D computer simulations of growing glioma and neovascular morphologies employing a multiscale mathematical model based on first principles and informed by experimental and clinical data, e.g., histopathology data transformed into model input parameters, and calibrated so that tumor morphology can be predicted beyond a purely empirical, observational approach [72]. The multiscale model predicts that glioma tissue structure and tumor invasiveness are significantly influenced by diffusion gradients in the microenvironment, as observed experimentally $[177,120,122,24,74]$ and in human patients [23]. These gradients may have a strong effect on glioma morphology [55, 135], and are hypothesized in the model to reciprocally influence a growing tumor's continuously evolving vasculature in complex ways. The 3 $\mathrm{D}$ model facilitates this study by calculating the chemotactic and haptotactic 
response of the blood vessels at the cell scale and its cumulative effect at the tumor scale.

\section{Conclusion}

The mathematical models and simulation results reviewed in this chapter aim to develop theories and numerical analyses to study cancer as a system across physical scales linking tumor morphology with cellular and microenvironmental characteristics through experimentally tested functional relationships. The central hypothesis underlying this research is inspired by an engineering approach to tumor lesions as complex micro-structured materials, where three-dimensional tissue architecture ("morphology") and dynamics are coupled in complex, nonlinear ways to cell phenotype, and this to molecular properties (e.g., genetics) and phenomena in the environment (e.g., hypoxia). These properties and phenomena act both as regulators of morphology and as determinants of invasion potential by controlling cell proliferation and migration mechanisms $[78,187,198]$. The importance of this close connection between tumor morphology and the underlying cellular/molecular scale is that it could allow observable properties of a tumor (e.g., morphology) to be used to understand the underlying cellular physiology and predict invasive behavior through mathematical modeling. Tumor morphology could also serve as a clinical prognostic factor because it may indicate the potential to respond to treatment, for instance, an indication of hypoxia could adversely affect oxygen-dependent such as radiation therapy and some chemotherapies. This approach opens the possibility of using mathematical modeling to design novel therapeutic strategies in which the microenvironment and cellular factors are manipulated with the aim of both imposing compact morphology and reducing tumor invasion - an outcome that would benefit cancer therapy by improving local tumor control through surgery or radiation.

The models also strive to provide a more comprehensive understanding of the molecular and environmental bases of cellular diversity and adaptation by describing the complex interactions among tumor cells and their microenvironment $[187,198]$. This approach is expected to improve current cancer modeling efforts because a multiscale approach model connects previous work focused on specific scales and specific processes (e.g., single cell motion) by performing 3 -dimensional simulations of in vivo tumors. This methodology further allows the possibility to see beyond the current reductionist picture of invasion and migration $[78,111,187,198,204,117,206,64,179,75,77,53,172]$, with the goal to enable prediction of disease progression and treatment response based on patient-specific tumor characteristics. 


\section{References}

1. Abbott, R.G., Forrest, S., Pienta, K.J.: Simulating the hallmarks of cancer. Artif. Life, 12, 617-634 (2006)

2. Acker, H.: Spheroids in cancer research: methods and perspectives. SpringerVerlag, Berlin and New York (1984)

3. Adam, J.: General aspects of modeling tumor growth and the immune response. In: Adam, J., Bellomo, N. (eds), A Survey of Models on Tumor Immune Systems Dynamics. Birkhauser, Boston, MA (1996)

4. Alarcón, T., Byrne, H.M., Maini, P.K.: A cellular automaton model for tumour growth in inhomogeneous environment. J. Theor. Biol., 225, 257-274 (2003)

5. Ambrosi, D., Guana, F.: Stress-modulated growth. Math. Mech. Solids, doi:10.1177/1081286505059739 (in press)

6. Ambrosi, D., Mollica, F.: On the mechanics of a growing tumor. Int. J. Eng. Sci., 40, 1297-1316 (2002)

7. Ambrosi, D., Preziosi, L.: On the closure of mass balance models for tumor growth. Math. Mod. Meth. Appl. Sci., 12, 737-754 (2002)

8. Andersen, H., Mejlvang, J., Mahmood, S., Gromova, I., Gromov, P., Lukanidin, E., Kriajevska, M., Mellon, J.K., Tulchinsky, E.: Immediate and delayed effects of E-cadherin inhibition on gene regulation and cell motility in human epidermoid carcinoma cells. Mol. Cell. Biol., 25, 9138-9150 (2005)

9. Anderson A, Zheng X, Cristini V.: Adaptive unstructured volume remeshing-I: The method. J. Comput. Phys., 208, 616-625 (2005)

10. Anderson, A.R.A.: A hybrid mathematical model of solid tumour invasion: the importance of cell adhesion. IMA Math. Appl. Med. Biol., 22, 163-186 (2005)

11. Anderson, A.R.A., Chaplain, M.A.J.: Continuous and discrete mathematical models of tumor-induced angiogenesis. Bull. Math. Biol., 60, 857-900 (1998)

12. Anderson, A.R.A. Chaplain, M.A.J., Newman, E.L., Steele, R.J.C., Thompson, A.M.: Mathematical modeling of tumour invasion and metastasis, J. Theor. Med., 2, 129-154 (2000)

13. Anderson, A.R.A., Weaver, A.M., Cummings, P.T., Quaranta, V.: Tumor Morphology and Phenotypic Evolution Driven by Selective Pressure from the Microenvironment. Cell, 127, 905-915 (2006)

14. Anderson, A.R.A., Chaplain, M.A.J., Lowengrub, J.S., Macklin, P., McDougall, S.: Nonlinear Simulation of Tumour Invasion and Angiogenesis. Bull. Math. Biol., (in preparation)

15. Anderson, D.M., McFadden, G.B., Wheeler, A.A.: Diffuse interface methods in fluid mechanics. Ann. Rev. Fluid Mech., 30, 139-165 (1998)

16. Araujo, R.P., McElwain, D.L.S.: A history of the study of solid tumor growth: the contribution of mathematical modeling. Bull. Math. Biol., 66, 1039-1091 (2004)

17. Araujo, R.P., McElwain, D.L.S.: A linear-elastic model of anisotropic tumor growth. Eur. J. Appl. Math., 15, 365-384 (2004)

18. Araujo, R.P., McElwain, D.L.S.: A mixture theory for the genesis of residual stresses in growing tissues II: solutions to the biphasic equations for a multicell spheroid. SIAM J. Appl. Math., 66, 447-467 (2005)

19. Augustin, H.G.: Tubes, branches, and pillars: The many ways of forming a new vasculature. Circ. Research, 89, 645-647 (2001) 
20. Bardelli, A., Basile, M.L., Audero, E., Giordano, S., Wennström, S., Ménard, S., Comoglio, P.M., Ponzetto, C.: Concomitant activation of pathways downstream of Grb2 and PI 3-kinase is required for MET-mediated metastasis. Oncogene, 18, 1139-1146 (1999)

21. Batchelor, G.: An Introduction to Fluid Dynamics. Cambridge University Press, Cambridge (1967)

22. Bauer, T.W., Liu, W.B., Fan, F., Camp, E.R., Yang, A., Somcio, R.J., Bucana, C.D., Callahan, J., Parry, G.C., Evans, D.B., Boyd, D.D., Mazar, A.P., Ellis, L.M.: Targeting of urokinase plasminogen activator receptor in human pancreatic carcinoma cells inhibits c-met- and insulin-like growth factor-1 receptormediated migration and invasion and orthotopic tumor growth in mice. Cancer Res., 65, 7775-7781 (2005)

23. Bearer, E.L., Cristini, V.: Computational modeling identifies morphologic predictors of tumor invasion. Science, (in review)

24. Bello, L., Lucini, V., Costa, F., Pluderi, M., Giussani, C., Acerbi, F., Carrabba, G., Pannacci, M., Caronzolo, D., Grosso, S., Shinkaruk, S., Colleoni, F., Canron, X., Tomei, G., Deleris, G., Bikfalvi, A.: Combinatorial Administration of Molecules That Simultaneously Inhibit Angiogenesis and Invasion Leads to Increased Therapeutic Efficacy in Mouse Models of Malignant Glioma. Clin. Cancer Res., 10, 4527-4537 (2004)

25. Bellomo, N., Preziosi, L.: Modelling and mathematical problems related to tumor evolution and its interaction with the immune system. Math. Comput. Modelling, 32, 413-542 (2000)

26. Bellomo, N., de Angelis, E., Preziosi, L.: Multiscale modelling and mathematical problems related to tumor evolution and medical therapy. J. Theor. Med., 5, 111-136 (2003)

27. Berger, M., Colella, P.: Local adaptive mesh refinement for shock hydrodynamics. J. Comp. Phys., 82, 64-84 (1989)

28. Bernsen, H.J.J.A., Van der Kogel, A.J.: Antiangiogenic therapy in brain tumor models. J. Neuro-oncology, 45, 247-255 (1999)

29. Bloemendal, H.J., Logtenberg, T., Voest, E.E.: New strategies in anti-vascular cancer therapy. Euro. J. Clinical Investig., 29, 802-809 (1999)

30. Boccaccio, C., Andò, M., Tamagnone, L., Bardelli, A., Michieli, P., Battistini, C., Comoglio, P.M.: Induction of epithelial tubules by growth factor HGF depends on the STAT pathway. Nature, 391, 285-288 (1998)

31. Brandt, A.: Multi-level adaptive solutions to boundary-value problems, Math. Comput., 31, 333-390 (1977)

32. Breward, C., Byrne, H., Lewis, C.: A multiphase model describing vascular tumour growth. Bull. Math. Biol., 65, 609-640 (2003)

33. Brizel, D.M., Scully, S.P., Harrelson, J.M., Layfield, L.J., Bean, J.M., Prosnitz, L.R., Dewhirst, M.W.: Tumor oxygenation predicts for the likelihood of distant metastases in human soft tissue sarcoma. Cancer Res., 56, 941-943 (1996)

34. Bullitt, E., Zeng, D., Gerig, G., Aylward, S., Joshi, S., Smith, J.K., Lin, W., Ewend, M.G.: Vessel tortuosity and brain tumor malignance: a blinded study. Acad. Radiol., 12, 1232-1240 (2005)

35. Bussolino, F., Arese, M., Audero, E., Giraudo, E., Marchiò, S., Mitola, S., Primo, L., Serini, G.: Biological aspects of tumour angiogenesis. In: Cancer Modelling and Simulation. Chapman and Hall/CRC, London, (2003)

36. Byrne, H.M.: The importance of intercellular adhesion in the development of carcinomas. IMA J. Math. Med. Biol., 14, 305-323 (1997) 
37. Byrne, H.M., Alarcón, T., Owen, M.R., Webb, S.D., Maini, P.K.: Modeling aspects of cancer dynamics: a review. Philos. Trans. R. Soc. A, 364, 15631578 (2006)

38. Byrne, H.M., Chaplain, M.A.J.: Growth of nonnecrotic tumors in the presence and absence of inhibitors. Mathl. Biosci., 130, 151-181 (1995)

39. Byrne, H.M., Chaplain, M.A.J.: Growth of necrotic tumors in the presence and absence of inhibitors. Math. Biosci., 135, 187-216 (1996)

40. Byrne, H.M., Chaplain, M.A.J.: Modelling the role of cell-cell adhesion in the growth and development of carcinomas. Math. Comput. Model., 24, 117 (1996)

41. Byrne, H.M., Chaplain, M.A.J.: Free boundary value problems associated with the growth and development of multicellular spheroids. Eur. J. Appl. Math., 8, 639-658 (1997)

42. Byrne, H.M., Matthews, P.: Asymmetric growth of models of avascular solid tumors: exploiting symmetries. IMA J. Math. Appl. Med. Biol., 19, 1-29 (2002)

43. Byrne, H., Preziosi, L.: Modelling solid tumour growth using the theory of mixtures. Math. Med. Biol., 20, 341-366 (2003)

44. Cairns, R.A., Kalliomaki, T., Hill, R.P.: Acute (cyclic) hypoxia enhances spontaneous metastasis of KHT murine tumors. Cancer Res., 61, 8903-8908 (2001)

45. Carmeliet, P., Jain, R.K.: Angiogenesis in cancer and other diseases. Nature, 407, 249-257 (2000)

46. Chaplain, M.A.J.: Avascular growth, Angiogenesis and Vascular growth in Solid Tumours: The Mathematic Modelling of the Stages of Tumour Development. Mathl. Comput. Modelling, 23, 47-87 (1996)

47. Chaplain, M.A.J.: Pattern formation in cancer. In: Chaplain, M.A.J., Singh, G.D., MacLachlan, J.C. (eds), On Growth and Form: Spatio-Temporal Pattern Formation in Biology. Wiley Series in Mathematical and Computational Biology. Wiley, New York, NY (2000)

48. Chaplain, M.A.J., Anderson, A.: Mathematical modelling of tissue invasion. In: Cancer Modelling and Simulation. Chapman and Hall/CRC, London (2003)

49. Chaplain, M.A.J., Ganesh, M., Graham, I.G.: Spatio-temporal pattern formation on spherical surfaces: numerical simulation and application to solid tumour growth. J. Math. Biol., 42, 387-423 (2001)

50. Chaplain, M.A.J., Graziano, L., Preziosi, L.: Mathematical modelling of the loss of tissue compression responsiveness and its role in solid tumour development. Math. Med. Biol., 23, 192-229 (2006)

51. Chaplain, M.A.J., Lolas, G.: Mathematical modeling of cancer cell invasion of tissue: The role of the urokinase plasminogen activation system. Math. Models Meth. Appl. Sci., 15, 1685-1734 (2005)

52. Chaplain, M.A.J., Stuart, A.: A model mechanism for the chemotactic response of endothelial cells to tumor angiogenesis factor. IMA J. Math. Appl. Med. Biol., 10, 149-168 (1993)

53. Condeelis, J., Singer, R.H., Segall, J.E.: The great escape: When cancer cells hijack the genes for chemotaxis and motility. Annu. Rev. Cell Dev. Biol., 21, 695-718 (2005)

54. Cristini, V., Blawzdziewicz, J., Loewenberg, M.: An adaptive mesh algorithm for evolving surfaces: Simulations of drop breakup and coalescence, J. Comput. Phys., 168, 445-463 (2001) 
55. Cristini, V., Frieboes, H.B., Gatenby, R., Caserta, S., Ferrari, M., Sinek, J.: Morphological instability and cancer invasion. Clin. Cancer Res., 11, 67726779 (2005)

56. Cristini, V., Gatenby, R., Lowengrub, J.: Multidisciplinary studies of tumor invasion and the role of the microenvironment. NIH 1R01CA127769-01., (2006)

57. Cristini, V., Li, X., Lowengrub, J., Wise, S.: Solid tumor growth: Mixture models., (in preparation)

58. Cristini, V., Lowengrub, J.: Three-dimensional crystal growth. I. Linear analysis and self-similar evolution. J. Crystal Growth, 240, 267-276 (2002)

59. Cristini, V., Lowengrub, J.S., Nie, Q.: Nonlinear simulation of tumor growth. J. Math. Biol., 46, 191-224 (2003)

60. Date, K., Matsumoto, K., Kuba, K., Shimura, H., Tanaka, M., Nakamura, T.: Inhibition of tumor growth and invasion by a four-kringle antagonist (HGF/NK4) for hepatocyte growth factor. Oncogene, 17, 3045-3054 (1998)

61. DeJaeger, K., Kavanagh, M.C., Hill, R.: Relationship of hypoxia to metastatic ability in rodent tumors. Br. J. Cancer, 84, 1280-1285 (2001)

62. Derycke, L., Van Marck, V., Depypere, H., Bracke, M.: Molecular targets of growth, differentiation, tissue integrity, and ectopic cell death in cancer cells. Cancer Biother. Radiopharm., 20, 579-588 (2005)

63. Eble, J.A., Haier, J.: Integrins in cancer treatment. Curr. Cancer Drug Targets, 6, 89-105 (2006)

64. Elvin, P., Garner, A.P.: Tumour invasion and metastasis: challenges facing drug discovery. Curr. Opin. Pharmacol., 5, 374-381 (2005)

65. Enam, S.A., Rosenblum, M.L., Edvardsen, K.: Role of extracellular matrix in tumor invasion: migration of glioma cells along fibronectinpositive mesenchymal cell processes. Neurosurgery, 42, 599-608 (1998)

66. Erler, J.T., Bennewith, K.L., Nicolau, M., Dornhoefer, N., Kong, C., L, Q.-T., Chi, J.-T.A., Jeffrey, S.S., Siaccia, A.J.: Lysyl oxidase is essential for hypoxiainduced metastasis. Nature, 440, 1222-1226 (2006)

67. Esteban, M.A., Maxwell, P.H.: HIF, a missing link between metabolism and cancer. Nature Med., 11, 1047-1048 (2005)

68. Fallenstein, G.T., Hulce, V.D., Melvin, J.W.: Dynamic mechanical properties of human brain tissue. J. Biomechanics, 2, 217-226 (1969)

69. Festjens, N., Vanden Berghe, T., Vandenabeele, P.: Necrosis, a well orchestrated form of cell demise: signalling cascades, important mediators and concomitant immune response. Biochim. Biophys. Acta, 1757, 1371-1387 (2006)

70. Forsythe, J.A., Jiang, B.H., Iyer, N.V., Agani, F., Leung, S.W., Koos, R.D., Semenza, G.L.: Activation of vascular endothelial growth factor gene transcription by hypoxia-inducible factor 1. Mol. Cell. Biol., 16, 4604-13 (1996)

71. Freyer, J.P.: Role of necrosis in regulating the growth saturation of multicellular spheroids. Cancer Res., 48, 2432-2439 (1988)

72. Frieboes, H.B., Lowengrub, J.S., Wise, S., Zheng, X., Macklin, P., Bearer, E.L., Cristini, V.: Computer simulation of glioma growth and morphology. NeuroImage, 37, S59-S70 (2007)

73. Frieboes, H.B., Wise, S.M., Lowengrub, J.S., Cristini, V.: Three dimensional diffuse-interface simulation of multispecies tumor growth-II: investigation of tumor invasion. Bull. Math. Biol., (in review)

74. Frieboes, H.B., Zheng, X., Sun, C.-H., Tromberg, B., Gatenby, R., Cristini, V.: An integrated computational/experimental model of tumor invasion. Cancer Res., 66, 1597-1604 (2006) 
75. Friedl, P.: Prespecification and plasticity: shifting mechanisms of cell migration. Curr. Opin. Cell Biol., 16, 14-23 (2004)

76. Friedl, P., Brocker, E., Zanker, K.: Integrins, cell matrix interactions and cell migration strategies: fundamental differences in leukocytes and tumor cells. Cell Adhe. Commun., 6, 225 (1998)

77. Friedl, P., Hegerfeldt, Y., Tilisch, M.: Collective cell migration in morphogenesis and cancer. Int. J. Dev. Biol., 48, 441-449 (2004)

78. Friedl, P., Wolf, A.: Tumor cell invasion and migration: diversity and escape mechanisms. Nat. Rev. Cancer, 3, 362-374 (2003)

79. Friedman, A.: A hierarchy of cancer models and their mathematical challenges, Discrete Cont. Dyn. Systems Ser. B, 4, 147-159 (2004)

80. Friedman, A., Reitich, F.: Analysis of a mathematical model for the growth of tumors. J. Math. Biol., 38, 262 (1999)

81. Friedman, A., Reitich, F.: On the existence of spatially patterned dormant malignancies in a model for the growth of non-necrotic vascular tumors. Math. Models Meth. Appl. Sci., 11, 601-625 (2001)

82. Galaris, D., Barbouti, A., Korantzopoulos, P.: Oxidative Stress in Hepatic Ischemia- Reperfusion Injury: The Role of Antioxidants and Iron Chelating Compounds. Current Pharma. Design, 12, 2875-2890 (2006)

83. Garber, K.: Energy boost: the Warburg effect returns in a new theory of cancer. JNCI, 96, 1805-1806 (2004)

84. Garcke, H., Nestler, B. and Stinner, B.: A diffuse interface model for alloys with multiple components and phases, SIAM J. Appl. Math., 64, 775-799 (2004)

85. Gatenby, R., Gawlinski, E.: A reaction-diffusion model of cancer invasion. Cancer Res., 56, 5745 (1996)

86. Gatenby, R.A., Gawlinski, E.T.: The glycolytic phenotype in carcinogenesis and tumor invasion: insights through mathematical models. Cancer Res., 63, 3847-3854 (2003)

87. Gatenby, R.A., Gawlinski, E.T., Gmitro, A.F., Kaylor, B., Gillies, R.J.: AcidMediated Tumor Invasion: a Multidisciplinary Study. Cancer Res., 66, 5216$5223(2006)$

88. Graeber, T.G., Osmanian, C., Jacks, T., Housman, D.E., Koch, C.J., Lowe, S.W., Giaccia, A.J.: Hypoxia-mediated selection of cells with diminished apoptotic potential in solid tumours. Nature, 379, 88-91 (1996)

89. Greenspan, H.P.: Models for the Growth of a Solid Tumor by diffusion. Stud. Appl. Math. LI, 4, 317-340 (1972)

90. Greenspan, H.P.: On the growth and stability of cell cultures and solid tumors. J. Theor. Biol., 56, 229-242 (1976)

91. Harris, A.L.: Hypoxia-a key regulatory factor in tumor growth. Nature Rev. Cancer 2, 38-47 (2002)

92. Hashizume, H., Baluk, P., Morikawa, S., McLean, J.W., Thurston, G., Roberge S., Jain, R.K., McDonald, D.M.: Openings between defective endothelial cells explain tumor vessel leakiness. American J. Pathology, 156, 1363-80 (2000)

93. Hayot, C., Debeir, O., Van Ham, P., Van Damme, M., Kiss, R., Decaestecker, C.: Characterization of the activities of actin-affecting drugs on tumor cell migration. Toxicology Appl. Pharma., 211, 30-40 (2006)

94. Helmlinger, G., Yuan, F., Dellian, M., Jain, R.K.: Interstitial pH and pO2 gradients in solid tumors in vivo: high-resolution measurements reveal a lack of correlation. Nat. Med., 3, 177-182 (1997) 
95. Höckel, M., Schlenger, K., Aral, B., Mitze, M., Schaffer, U., Vaupel, P.: Association between tumor hypoxia and malignant progression in advanced cancer of the uterine cervix. Cancer Res., 56, 4509-4515 (1996)

96. Höckel, M., Vaupel, P.: Tumor hypoxia: definitions and current clinical, biologic, and molecular aspects. J. Natl. Cancer Inst., 93, 266-276 (2001)

97. Hogea, C.S., Murray, B.T., Sethian, J.A.: Simulating complex tumor dynamics from avascular to vascular growth using a general level-set method. J. Math. Biol., 53, 86-134 (2006)

98. Holash, J. Maisonpierre, P.C., Compton, D., Boland, P., Alexander, C.R., Zagzag, D., Yancopoulos, G.D., Wiegand, S.J.: Vessel cooption, regression, and growth in tumors mediated by angiopoietins and VEGF. Science, 284, 19941998 (1999)

99. Holash, J., Wiegand, S.J., Yancopoulos, G.D.: New model of tumor angiogenesis: dynamic balance between vessel regression and growth mediated by angiopoietins and VEGF. Oncogene, 18, 5356-5362 (1999)

100. Huang, Q., Shen, H.M., Ong, C.N.: Emodin inhibits tumor cell migration through suppression of the phosphatidylinositol 3-kinase-Cdc42/Rac1 pathway. Cell. Molecular Life Sci., 62, 1167-1175 (2005)

101. Jacqmin, D.: Calculation of two-phase Navier-Stokes flows using phase-field modeling. J. Comp. Phys., 155, 96-127 (1999)

102. Jain, R.K.: Determinants of tumor blood flow: a review. Cancer Res., 48, 26412658 (1988)

103. Jain, R.K.: Physiological Barriers to Delivery of Monoclonal Antibodies and Other Macromolecules in Tumors. Cancer Res. (Suppl.), 50, 814s-819s (1990)

104. Jain, R.K: Delivery of molecular and cellular medicine to solid tumors. J. Control. Release, 53, 49-67 (1998)

105. Jain, R.K.: Normalizing tumor vasculature with anti-angiogenic therapy: a new paradigm for combination therapy. Nat. Med., 7, 987-989 (2001)

106. Jain, R.K.: Delivery of molecular medicine to solid tumors: lessons from in vivo imaging of gene expression and function. J. Controlled Release, 74, 7-25 (2001)

107. Jain, R.K.: Molecular regulation of vessel maturation. Nature Med., 9, 685-693 (2003)

108. Jones, A.F., Byrne, H.M., Gibson, J.S., Dold, J.W.: A mathematical model of the stress induced during avascular tumor growth. J. Math. Biol., 40, 473-499 (2000)

109. Kansal, A.A., Torquato, S., Harsh IV, G.R., Chiocca, E.A., Deisboeck, T.S.: Simulated brain tumor growth dynamics using a 3-D cellular automaton. J. Theor. Biol., 203, 367-382 (2000)

110. Kaur, B., Khwaja, F.W., Severson, E.A., Matheny, S.L., Brat, D.J., VanMeir, E.G.: Hypoxia and the hypoxia-inducible-factor pathway in glioma growth and angiogenesis. Neuro-oncol., 7, 134-153 (2005)

111. Keller, P.J., Pampaloni, F., Stelzer, E.H.K.: Life sciences require the third dimension. Curr. Op. Cell Biol., 18, 117-124 (2006)

112. Kim, J.S., Kang, K., Lowengrub, J.S.: Conservative multigrid methods for Cahn-Hilliard fluids. J. Comp. Phys., 193, 511-543 (2004)

113. Kim, J.S., Kang, K., Lowengrub, J.S.: Conservative multigrid methods for ternary Cahn-Hilliard systems. Comm. Math. Sci., 12, 53-77 (2004)

114. Kim, J.S., Lowengrub, J.S.: Phase field modeling and simulation of three-phase flows. Int. Free Bound., 7, 435 (2005) 
115. Kloner, R.A., Jennings, R.B.: Consequences of brief ischemia: stunning, preconditioning, and their clinical implications: part 1. Circulation, 104, 2981-2989 (2001)

116. Konopleva, M., Zhao, S.R., Hu, W., Jiang, S.W., Snell, V., Weidner, D., Jackson, C.E., Zhang, X., Champlin, R., Estey, E., Reed, J.C., Andreeff, M.: The anti-apoptotic genes Bcl-X-L and Bcl-2 are over- expressed and contribute to chemoresistance of non-proliferating leukaemic CD34 cells. Br. J. Haematol., 118, 521-534 (2002)

117. Kopfstein, L., Christofori, G.: Metastasis: cell-autonomous mechanisms versus contributions by the tumor microenvironment. Cell. Mol. Life Sci., 63, 449-468 (2006)

118. Kuiper, R.A.J., Schellens, J.H.M., Blijham, G.H., Beijnen, J.H., Voest, E.E.: Clinical research on antiangiogenic therapy. Pharmacol Res., 37, 1-16 (1998)

119. Kumar, M., Krishnan, G., Arumainanthan, U., Singh, K.: Endoscopic Excision of A Nasal Glioma. The Internet Journal of Otorhinolaryngology, 2 (2003)

120. Kunkel, P., Ulbricht, U., Bohlen, P., Brockmann, M.A., Fillbrandt, R., Stavrou, D., Westphal, M., Lamszus, K.: Inhibition of Glioma Angiogenesis and Growth in Vivo by Systemic Treatment with a Monoclonal Antibody against Vascular Endothelial Growth Factor Receptor-2. Cancer Res., 61, 6624-6628 (2001)

121. Lah, T.T., Alonso, M.B.D., Van Noorden, C.J.F.: Antiprotease therapy in cancer: hot or not? Exp. Op. Biol. Ther., 6, 257-279 (2006)

122. Lamszus, K., Kunkel, P., Westphal, M.: Invasion as limitation to antiangiogenic glioma therapy. Acta Neurochir. Suppl., 88, 169-177 (2003)

123. Lee, H., Lowengrub, J.S., Goodman, J.: Modeling pinchoff and reconnection in a Hele-Shaw cell I. The models and their calibration, Phys. Fluids, 14, 492-513 (2002)

124. Leo, P.H., Lowengrub, J.S., Jou, H.-J.: A diffuse interface model for elastically stressed solids. Acta Metall., 46, 2113-2130 (1998)

125. Less, J.R., Skalak, T.C., Sevick, E.M., Jain, R.K.: Microvascular architecture in a mammary carcinoma: branching patterns and vessel dimensions. Cancer Res., 51, 265-273 (1991)

126. Lester, R.D., Jo, M., Campana, W.M., Gonias, S.L.: Erythropoietin promotes MCF-7 breast cancer cell migration by an ERK/mitogenactivated protein kinase-dependent pathway and is primarily responsible for the increase in migration observed in hypoxia. J. Biol. Chem., 280, 39273-39277 (2005)

127. Li, X., Cristini, V., Nie, Q., Lowengrub, J.S.: Nonlinear three dimensional simulation of solid tumor growth. Disc. Cont. Dyn. Sys. B, 7, 581-604 (2007)

128. Lockett, J., Yin, S.P., Li, X.H., Meng, Y.H., Sheng, S.J.: Tumor suppressive maspin and epithelial homeostasis. J. Cell. Biochem., 97, 651-660 (2006)

129. Lowengrub, J.S., Macklin, P.: A Centimeter-Scale Nonlinear Model of Tumor Growth in Complex, Heterogeneous Tissues. J. Math. Biol., (in preparation)

130. Lowengrub, J.S., Truskinovsky, L.: Quasi-incompressible Cahn-Hilliard fluids and topological transitions. Proc. R. Soc. London A, 454, 2617-2654 (1998)

131. Lubarda, V., Hoger, A.: On the mechanics of solids with a growing mass. Int. J. Solids Structures, 39, 4627 (2002)

132. Macklin, P.: Numerical Simulation of Tumor Growth and Chemotherapy. MS thesis, University of Minnesota, Minnesota (2003)

133. Macklin, P., Lowengrub, J.S.: Evolving interfaces via gradients of geometrydependent interior Poisson problems: application to tumor growth. J. Comput. Phys., 203, 191-220 (2005) 
134. Macklin, P., Lowengrub, J.: An improved geometry-aware curvature discretization for level-set methods: Application to tumor growth, J. Comput. Phys., 215, 392-401 (2006)

135. Macklin, P., Lowengrub, J.: Nonlinear simulation of the effect of microenvironment on tumor growth. J. Theor. Biol., 245, 677-704 (2007)

136. Macklin, P., Lowengrub, J.: A new ghost cell/level set method for moving boundary problems: application to tumor growth. J. Scientific Comp., (in review)

137. Maher, E., Furnari, F., Bachoo, R., Rowitch, D., Louis, D., Cavenee, W., DePinho, R.: Malignant glioma: genetics and biology of a grave matter. Genes Dev., 15, 1311 (2001)

138. Mallett, D.G., de Pillis, L.G.: A cellular automata model of tumorimmune system interactions. J. Theor. Biol., 239, 334-350 (2006)

139. Mansury, Y., Kimura, M., Lobo, J., Deisboeck, T.S.: Emerging patterns in tumor systems: simulating the dynamics of multicellular clusters with an agentbased spatial agglomeration model. J. Theor. Biol., 219, 343-370 (2002)

140. McDougall, S.R., Anderson, A.R.A., Chaplain, M.A.J.: Mathematical modeling of dynamic adaptive tumour-induced angiogenesis: Clinical applications and therapeutic targeting strategies. J. Theor. Biol., 241, 564-589 (2006)

141. McDougall, S.R., Anderson, A.R.A., Chaplain, M.A.J., Sherratt, J.: Mathematical modelling of flow through vascular networks: implications for tumourinduced angiogenesis and chemotherapy strategies. Bull. Math. Biol., 64, 673$702(2002)$

142. McElwain, D.L.S., Morris, L.E.: Apoptosis as a volume loss mechanism in mathematical models of solid tumor growth. Math. Biosci., 39, 147-157 (1978)

143. McLean, G.W., Carragher, N.O., Avizienyte, E., Evans, J., Brunton, V.G., Frame, M.C.: The role of focal-adhesion kinase in cancer. A new therapeutic opportunity. Nat. Rev. Cancer, 5, 505-515 (2005)

144. Michieli, P., Basilico, C., Pennacchietti, S., Maffè, A., Tamagnone, L., Giordano, S., Bardelli, A., Comoglio, P.M.: Mutant Met mediated transformation is ligand-dependent and can be inhibited by HGF antagonists. Oncogene, 18, 5221-5231 (1999)

145. Montesano, R., Matsumoto, K., Nakamura, T. Orci, L.: Identification of a fibroblast-derived epithelial morphogen as hepatocyte growth factor. Cell, 67, 901-908 (1991)

146. Morotti, A., Mila, S., Accornero, P., Tagliabue, E., Ponzetto, C. K252a inhibits the oncogenic properties of Met, the HGF receptor. Oncogene, 21, 4885-4893 (2002)

147. Mueller-Klieser, W.: Multicellular spheroids: a review on cellular aggregates in cancer research. J. Cancer Res. Clin. Oncol., 113, 101-122 (1987)

148. Naganuma, H., Kimurat, R., Sasaki, A., Fukamachi, A., Nukui, H., Tasaka, K.: Complete remission of recurrent glioblastoma multiforme following local infusions of lymphokine activated killer cells. Acta Neurochir., 99, 157-160 (1989)

149. Natarajan, M., Hecker, T.P., Gladson, C.L.: Fak signaling in anaplastic astrocytoma and glioblastoma tumors. Cancer J. 9, 126-133 (2003)

150. Netti, P.A., Baxter, L.T., Boucher, Y., Skalak, R., Jain, R.K.: Time dependent behavior of interstitial fluid pressure in solid tumors: Implications for drug delivery. Cancer Res., 55, 5451-5458 (1995) 
151. Nor, J.E., Christensen, J., Liu, J., Peters, M., Mooney, D.J., Strieter, R.M., Polverini, P.J.: Up-Regulation of Bcl-2 in Microvascular Endothelial Cells Enhances Intratumoral Angiogenesis and Accelerates Tumor Growth. Cancer Res., 61, 2183-2188 (2001)

152. O'Connor, J.P.B., Jackson, A., Parker, G.J.M., Jayson, G.C.: DCE-MRI biomarkers in the clinical evaluation of antiangiogenic and vascular disrupting agents. Br. J. Cancer, 96, 189-195 (2007)

153. Ommaya, A.K.: Mechanical properties of tissues of the nervous system. J. Biomechanics, 1, 127-138 (1968).

154. Osher, S., Sethian, J.: Fronts propagating with curvature-dependent speed: algorithms based on Hamilton-Jacobi formulation. J. Comput. Phys., 79, 12 (1988)

155. Padera, T.P., Stoll, B.R., Tooredman,,J.B., Capen, D., di Tomaso, E., Jain, R.: Cancer cells compress intratumour vessels. Nature, 427, 695 (2004)

156. Page, D.L., Anderson, T.J., Sakamoto, G: In: Diagnostic Histopathology of the Breast Churchill Livingstone, New York (1987)

157. Paku, S.: Paweletz, N.: First step of tumor-related angiogenesis. Lab. Invest., 65, 334-346 (1991)

158. Patan, S., Tanda, S., Roberge, S., Jones, R.C., Jain, R.K., Munn, L.L.: Vascular morphogenesis and remodeling in a human tumor xenograft: blood vessel formation and growth after ovariectomy and tumor implantation. Circ. Research, 89, 732-739 (2001)

159. Paweletz, N., Knierim, M.: Tumor-related angiogenesis. Crit. Rev. Oncol. Hematol., 9, 197-242 (1989)

160. Pennacchietti, S., Michieli, P., Galluzzo, M., Giordano, S., Comoglio, P.: Hypoxia promotes invasive growth by transcriptional activation of the met protooncogene. Cancer Cell, 3, 347-361 (2003)

161. Plank, M.J., Sleeman, B.D.: A reinforced random walk model of tumour angiogenesis and anti-angiogenic strategies. Math. Med. Biol., 20, 135-181 (2003)

162. Plank, M.J., Sleeman, B.D.: Lattice and non-lattice models of tumour angiogenesis. Bull. Math. Biol., 66, 1785-1819 (2004)

163. Please, C.P., Pettet, G., McElwain, D.L.S.: A new approach to modeling the formation of necrotic regions in tumors. Appl. Math. Lett., 11, 89-94 (1998)

164. Polette, M., Gilles, C., de Bentzmann, S., Gruenert, D., Tournier, J.M., Birembaut, P.: Association of fibroblastoid features with the invasive phenotype in human bronchial cancer cell lines. Clin. Exp. Metastasis, 16, 105-112 (1998)

165. Postovit, L.M., Adams, M.A., Lash, G.E., Heaton, J.P., Graham, C.H.: Oxygen-mediated regulation of tumor cell invasiveness. Involvement of a nitric oxide signaling pathway. J. Biol. Chem., 277, 35730-35737 (2002)

166. Pouysségur, J., Dayan, F., Mazure, N.M.: Hypoxia signalling in cancer and approaches to enforce tumour regression. Nature, 441, 437-443 (2006)

167. Putz, E., Witter, K., Offner, S., Stosiek, P., Zippelius, A., Johnson, J., Zahn, R., Riethmüller, G., Pantel, K.: Phenotypic characteristics of cell lines derived from disseminated cancer cells in bone marrow of patients with solid epithelial tumors: establishment of working models for human micrometastases. Cancer Res., 59, 241-248 (1999)

168. Quaranta, V., Weaver, A.M., Cummings, P.T., Anderson, A.R.A.: Mathematical modeling of cancer: the future of prognosis and treatment. Clin. Chim. Acta, 357, 173-179 (2005) 
169. Ramakrishnan, S.: Department of Pharmacology, University of Minnesota. Personal communication., (2003)

170. Ramanathan, A., Wang, C., Schreiber, S.L.: Perturbational profiling of a cellline model of tumorigenesis by using metabolic measurements. Proc. Natl Acad. Sci., 102, 5992-5997 (2005)

171. Ravandi, F., Estrov, Z.: Eradication of leukemia stem cells as a new goal of therapy in leukemia. Clin. Cancer Res., 12, 340-344 (2006)

172. Ridley, A.J., Schwartz, M.A., Burridge, K., Firtel, R.A., Ginsberg, M.H., Borisy, G., Parsons, J.T., Horwitz, A.R.: Cell migration: Integrating signals from front to back. Science., 302, 1704-1709 (2003)

173. Rofstad, E., Halsør, E.: Hypoxia-associated spontaneous pulmonary metastasis in human melanoma xenographs: involvement of microvascular hotspots induced in hypoxic foci by interleukin. Br. J. Cancer, 86, 301-308 (2002)

174. Rofstad, E., Rasmussen, H., Galappathi, K., Mathiesen, B., Nilsen, K., Graff, B.A.: Hypoxia promotes lymph node metastasis in human melanoma xenografts by up-regulating the urokinase-type plasminogen activator receptor. Cancer Res., 62, 1847-1853 (2002)

175. Rohzin, J., Sameni, M., Ziegler, G., and Sloane, B.F.: Pericellular pH affects distribution and secretion of cathepsin B in malignant cells. Cancer Res., 54, 6517-6525 (1994)

176. Roose, T., Netti, P.A., Munn, L.L., Boucher, Y., Jain, R.: Solid stress generated by spheroid growth estimated using a linear poroelastic model. Microvasc. Res., 66, 204-212 (2003)

177. Rubenstein, J.L., Kim, J., Ozawa, T., Zhang, K., Westphal, M., Deen, D.F., Shuman, M.A.: Anti-VEGF antibody treatment of glioblastoma prolongs survival but results in increased vascular cooption. Neoplasia, 2, 306-314 (2000)

178. Saad, Y., Schultz, M.H. GMRES: A Generalized Minimal Residual Algorithm for Solving Nonsymmetric Linear Systems, SIAM J. Sci. Stat. Comput. 7, 856869 (1986)

179. Sahai, E.: Mechanisms of cancer cell invasion. Curr. Opin. Genet. Dev., 15, 87-96(2005)

180. Sakamoto, G.: Infiltrating carcinoma: major histological types. In: Page, D.L., Anderson, T.J. (eds), Diagnostic Histopathology of the Breast. ChurchillLivingstone, London, UK (1987)

181. Sanga, S., Sinek, J.P., Frieboes, H.B., Fruehauf, J.P., Cristini, V.: Mathematical modeling of cancer progression and response to chemotherapy. Expert. Rev. Anticancer Ther., 6, 1361-1376 (2006)

182. Sanga, S., Frieboes, H.B., Zheng, X., Bearer, E.L., Cristini V.: Predictive oncology: a review of multidisciplinary, multiscale in silico modeling linking phenotype, morphology and growth. NeuroImage., (in press)

183. Sanson, B.C., Delsanto, P.P., Magnano, M., Scalerandi, M.: Effects of anatomical constraints on tumor growth. Phys. Rev. E, 64, 21903ff (2002)

184. Schmeichel, K.L., Weaver, V.M., Bissel, M.J.: Structural cues from the tissue microenvironment are essential determinants of the human mammary epithelial cell phenotype. J. Mammary Gland Biol. Neoplasia, 3, 201-213 (1998)

185. Seftor, E.A., Meltzer, P.S., Kirshmann, D.A., D.A., Pe'er, J., Maniotis, A.J., Trent, J.M., Folberg, R., Hendrix, M.J.: Molecular determinants of human uveal melanoma invasion and metastasis. Clin. Exp. Metastasis, 19, 233-246 (2002) 
186. Shweiki, D., Itin, A., Soffer, D., Keshet, E.: Vascular endothelial growth factor induced by hypoxia may mediate hypoxia-initiated angiogenesis. Nature, 359, 843-845 (1992)

187. Sierra, A.: Metastases and their microenvironments: linking pathogenesis and therapy. Drug Resistance Updates, 8, 247-257 (2005)

188. Sinek, J., Frieboes, H., Zheng, X., Cristini, V.: Two-dimensional chemotherapy simulations demonstrate fundamental transport and tumor response limitations involving nanoparticles. Biomed. Microdev., 6, 197-309 (2004)

189. Skinner, S.A.: Microvascular architecture of experimental colon tumors in the rat. Cancer Res., 50, 2411-2417 (1990)

190. Steeg, P.S.: Angiogenesis inhibitors: motivators of metastasis? Nature Med., 9, 822-823 (2003)

191. Sun S., Wheeler, M.F., Obeyesekere, M., Patrick Jr., C.: Multiscale angiogenesis modeling using mixed finite element methods, Multiscale Model. Simul., 4, 1137-1167 (2005)

192. Sutherland, R.M.: Cell and Environment interactions in tumor microregions: the multicell spheroid model. Science, 240, 177-184 (1988)

193. Takano, S., Yoshii, Y., Kondo, S., Suzuki, H., Maruno, T., Shirai, S., Nose, T.: Concentration of vascular endothelial growth factor in the serum and tumor tissue of brain tumor patients. Cancer Res., 56, 2185-2190 (1996)

194. Tester, A. M., Ruangpani, N., Anderso, R. L. \& Thompson, E.W.: MMP-9 secretion and MMP-2 activation distinguish invasive and metastatic sublines of a mouse mammary carcinoma system showing epithelial-mesenchymal transition traits. Clin. Exp. Metastasis, 18, 553-560 (2000)

195. Tiller, W. Migration of a liquid zone through a solid. 3. J. Appl. Phys., 36, $261(1965)$

196. Truskey, G., Yuan, F., Katz, D.: Transport Phenomena in Biological Systems. Pearson Prentice Hall, Upper Saddle River, NJ. (2004)

197. Vajkoczy, P., Farhadi, M., Gaumann, A., Heidenreich, R., Erber, R., Wunder, A., Tonn, J.C., Menger, M.D., Breier, G.: Microtumor growth initiates angiogenic sprouting with simultaneous expression of VEGF, VEGF receptor-2, and angiopoietin-2. J. Clin. Invest., 109, 777-785 (2002)

198. van Kempen, LCLT; Ruiter, DJ; van Muijen, GNP; Coussens, LM.: The tumor microenvironment: a critical determinant of neoplastic evolution. Eur. J. Cell. Biol., 82, 539-548 (2003)

199. Vaupel, P., Haugland, H.K., Nicklee, T., Morrison, A.J., Hedley, D.W.: Hypoxia-inducible factor-1 alpha is an intrinsic marker for hypoxia in cervical cancer xenografts. Cancer Res., 61, 7394-7398 (2001)

200. Vaupel, P., Kallinowski, F., Okunieff, P.: Blood flow, oxygen and nutrient supply, and metabolic microenvironment of human tumours: a review. Cancer Res., 49, 6449-6465 (1989)

201. Wise, S.M., Kim, J., Lowengrub, J.: Solving the regularized, strongly anisotropic Cahn-Hilliard equation by an adaptive nonlinear multigrid method. J. Comput. Phys., doi:10.1016/j.jcp.2007.04.020 (in press)

202. Wise, S.M., Lowengrub, J.S., Frieboes, H.B., Cristini, V.: Three dimensional diffuse-interface simulation of multispecies tumor growth-I: Numerical. Method. Bull. Math. Biol., (in review)

203. Wise, S.M., Lowengrub, J.S., Kim, J.S., Johnson, W.C.: Efficient phase-field simulation of quantum dot formation in a strained heteroepitaxial film. Superlattices and Microstructures, 36, 293-304 (2004) 
204. Wolf, K., Friedl, P.: Molecular mechanisms of cancer cell invasion and plasticity. Br. J. Dermatology, 154, 11-15 (2006)

205. Wurzel, M., Schaller, C., Simon, M., Deutsch, A.: Cancer cell invasion of brain tissue: guided by a prepattern? J. Theor. Medicine, 6, 21-31 (2005)

206. Yamaguchi, H., Wyckoff, J., Condeelis, J.: Cell migration in tumors. Curr. Op. Cell Biol., 17, 559-564 (2005)

207. Yin, S.P., Lockett, J., Meng, Y.H., Biliran, H., Blouse, G.E., Li, X.H., Reddy, N., Zhao, Z.M., Lin, X.L., Anagli, J., Cher, M.L., Sheng, S.J.: Maspin retards cell detachment via a novel interaction with the urokinase-type plasminogen activator/urokinase-type plasminogen activator receptor system. Cancer Res., 66, 4173-4181 (2006)

208. Young, S.D., Hill, R.P.: Effects of reoxygenation of cells from hypoxic regions of solid tumors: anticancer drug sensitivity and metastatic potential. J. Natl. Cancer Inst., 82, 338-339 (1990)

209. Young, S.D., Marshall, R.S., Hill, R.P.: Hypoxia induces DNA overreplication and enhances metastatic potential of murine tumor cells. Proc. Natl. Acad. Sci. USA, textbf85, 9533-9537 (1988)

210. Yu, J., Rak, J., Coomber, B., Hicklin, D., Kerbel, R.: Effect of p53 status on tumor response to antiangiogenic therapy. Science, 295, 1526-1528 (2002)

211. Zagzag, D., Amirnovin, R.,Greco, M.A., Yee, H., Holash, J., Wiegand, S.J., Zabski, S., Yancopoulos, G.D., Grumet, M.: Vascular apoptosis and involution in gliomas precede neovascularization: a novel concept for glioma growth and angiogenesis. Lab Invest., 80, 837-849 (2000)

212. Zheng, X., Wise, S.M., Cristini, V.: Nonlinear simulation of tumor necrosis, neo-vascularization and tissue invasion via an adaptive finite element/level set method. Bull. Math. Biol., 67, 211-259 (2005)

\section{Figures}




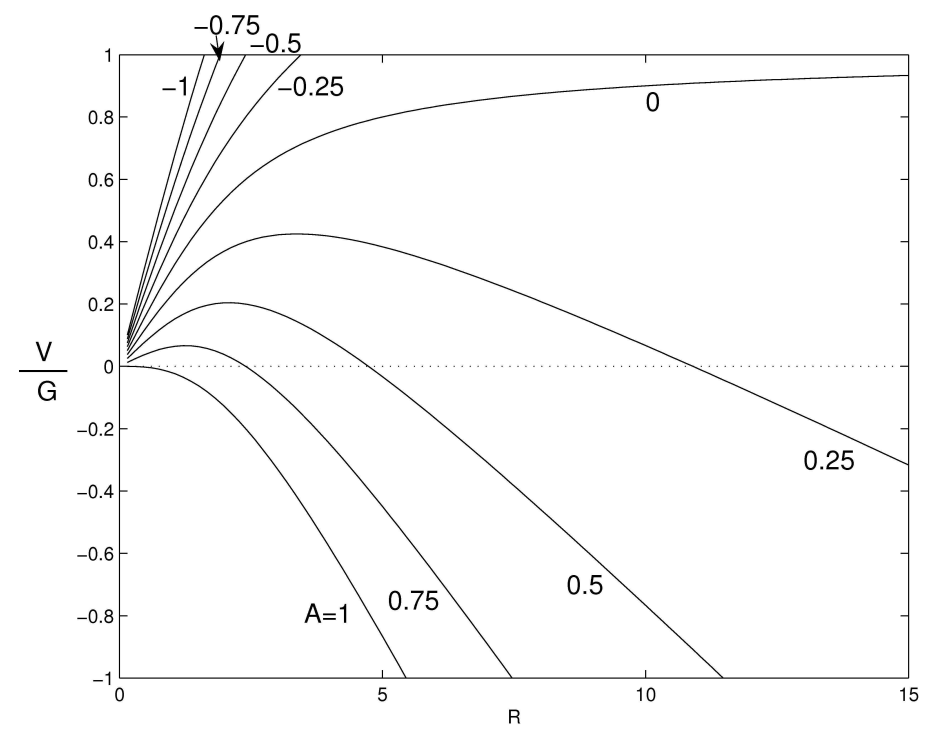

Fig. 1. Rescaled rate of growth $G^{-1} V$ from (7) as a function of rescaled tumor radius $R$ for radially symmetric tumor growth and $d=2 ; A$ labelled. Reprinted from Journal of Mathematical Biology, Cristini et al. Vol. 46, p. 195, Copyright 2003 Springer. With kind permission of Springer Science and Business Media. 

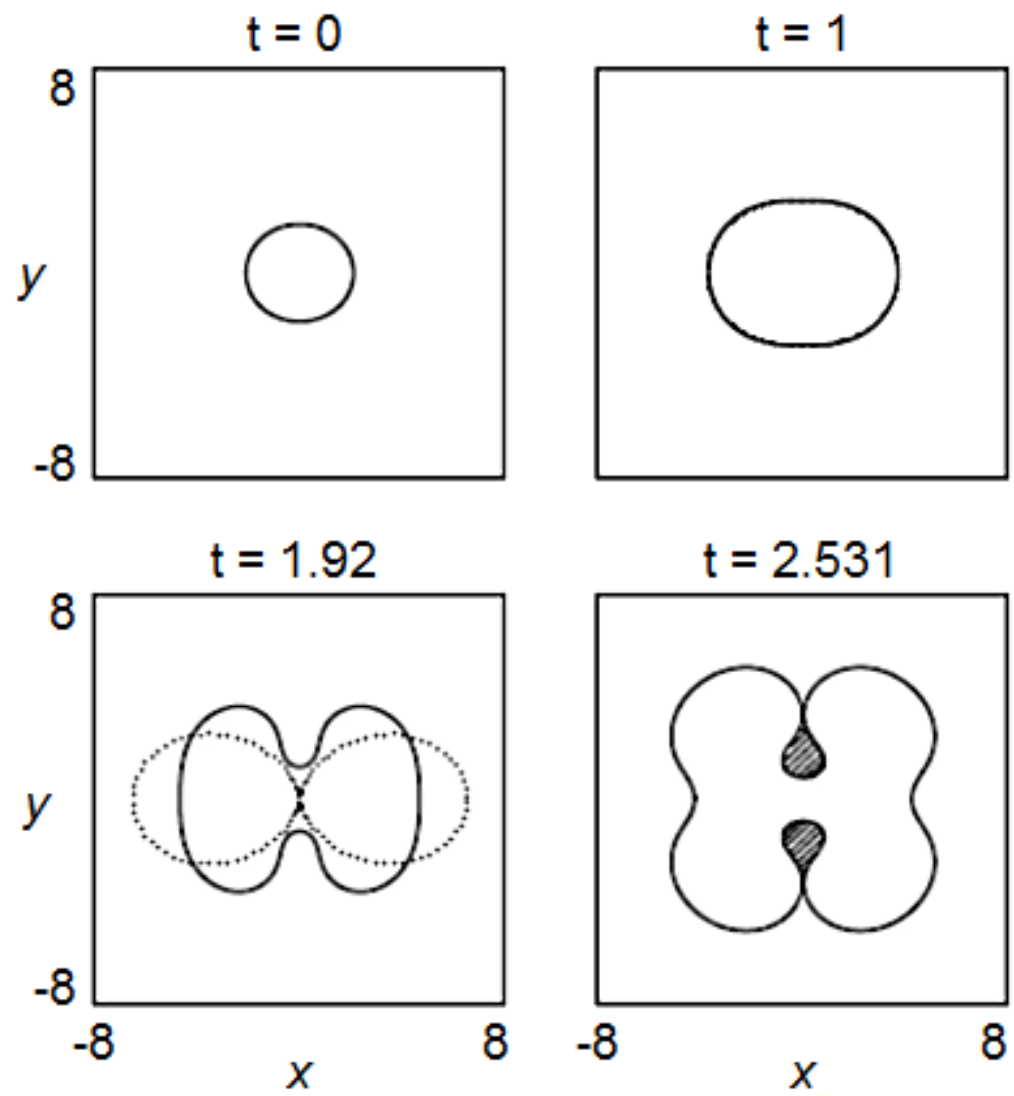

Fig. 2. Evolution of the tumor surface in the low-vascularization regime, for $d=2$, $A=0.5, G=20$, and initial tumor surface as in equation (12). Dotted lines: solution from linear analysis; solid: solution from a nonlinear calculation with time step $\triangle t=10^{-3}$ and a number of marker points $N=1024$, reset, after time $t=2.51$, to $\triangle t=10^{-4}$ and $N=2048$. Reprinted from Journal of Mathematical Biology, Cristini et al. Vol. 46, p. 202, Copyright 2003 Springer. With kind permission of Springer Science and Business Media. 


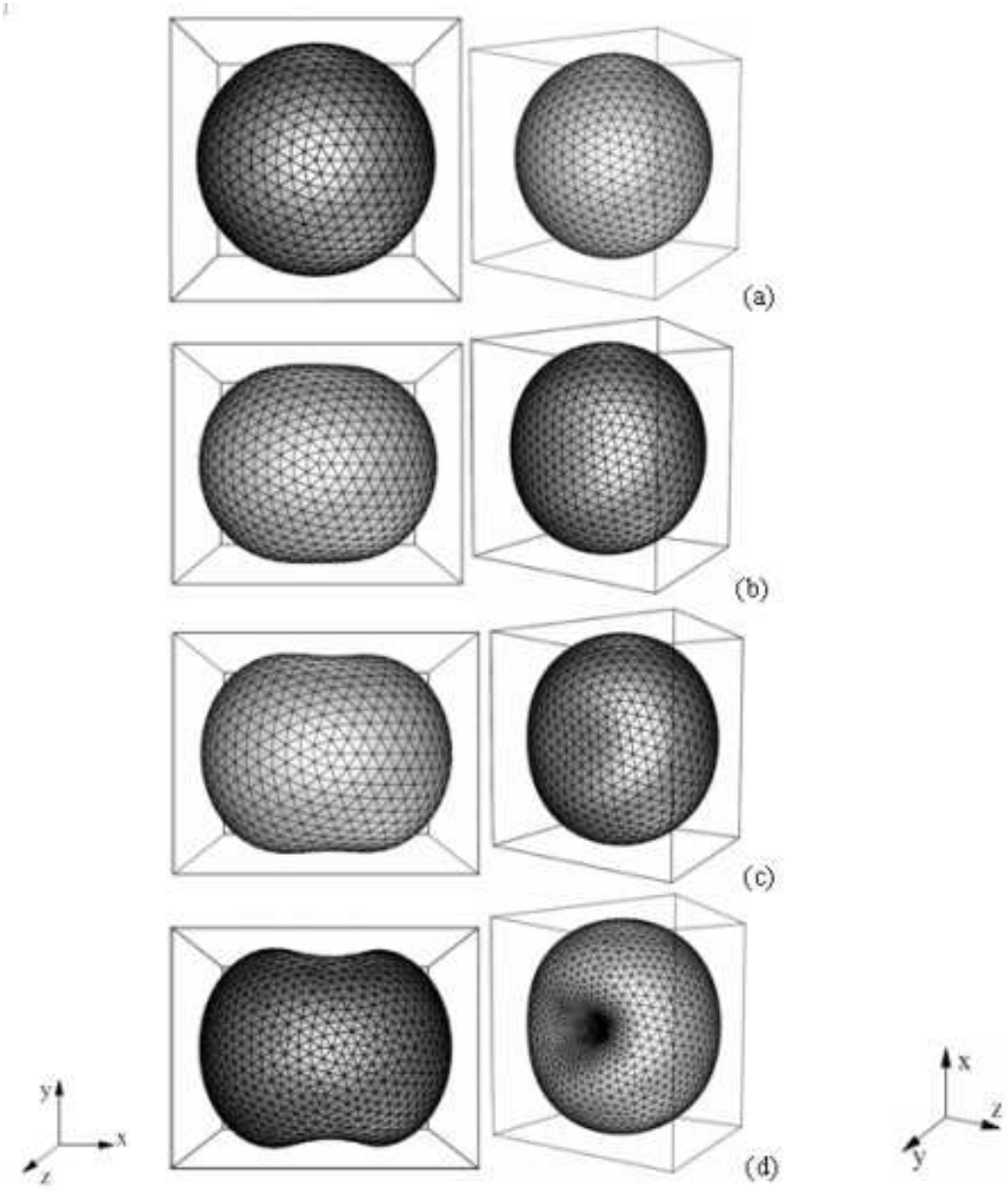

Fig. 3. Evolution of the tumor surface in the low vascularization regime, $A=0.5$, $G=20$, and initial tumor surface as in (13). (a) $t=0, \bar{\delta}=0.0137, S=3.0$ (b) $t=2.21, \bar{\delta}=0.12, S=4.732$ (c) $t=2.42, \bar{\delta}=0.2, S=4.745$ (d) $t=2.668$, $\bar{\delta}=0.496, S=4.781$. Reprinted with permission from Discrete and Continuous Dynamical Systems - Series B, Li et al., Vol. 7, p. 599. Copyright 2007 American Institute of Mathematical Sciences. 


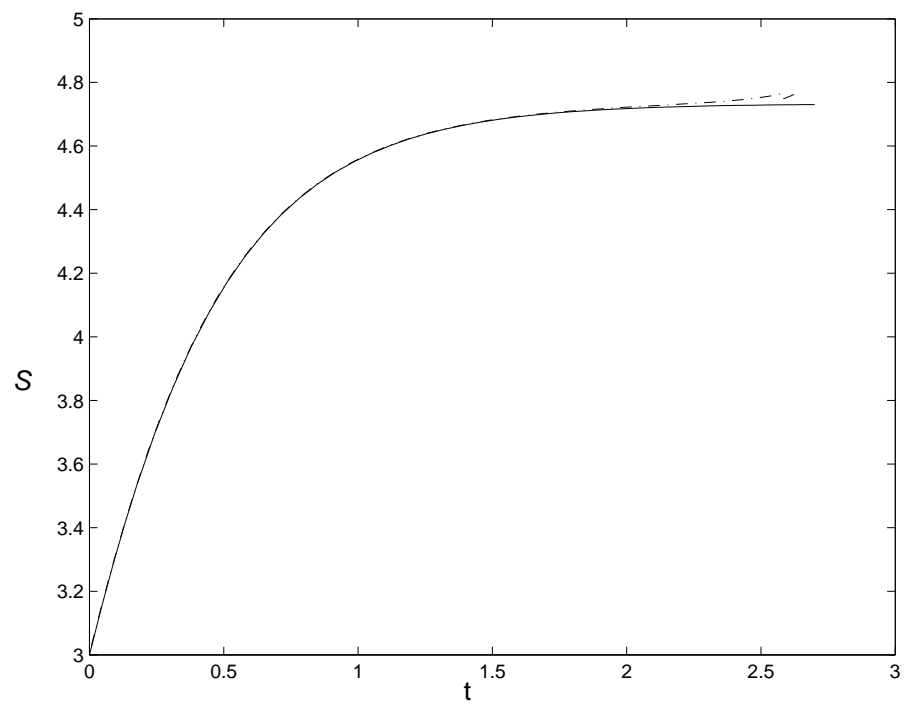

(a)

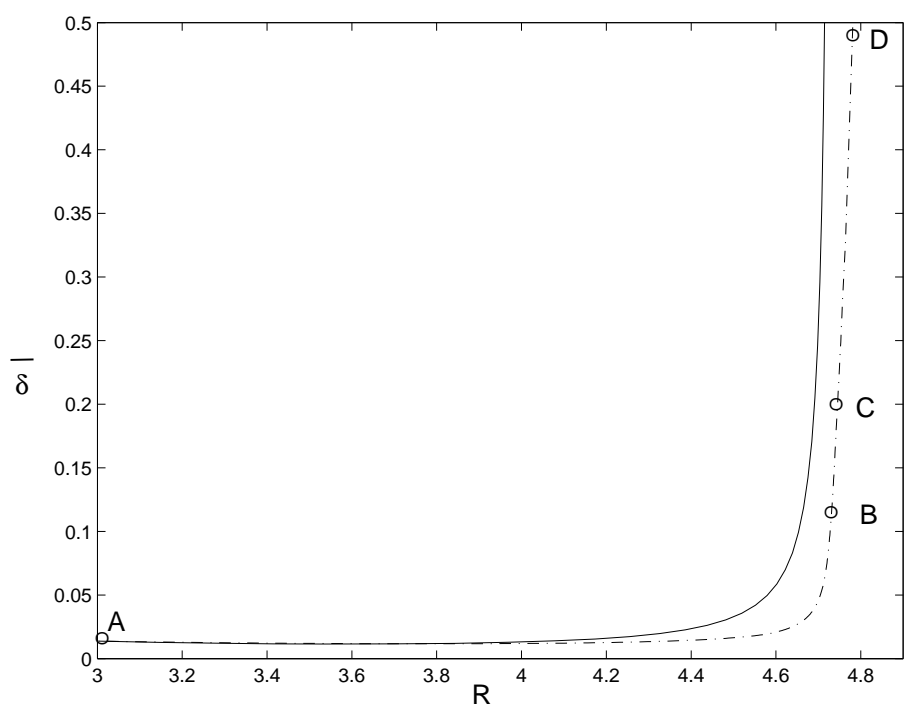

(b)
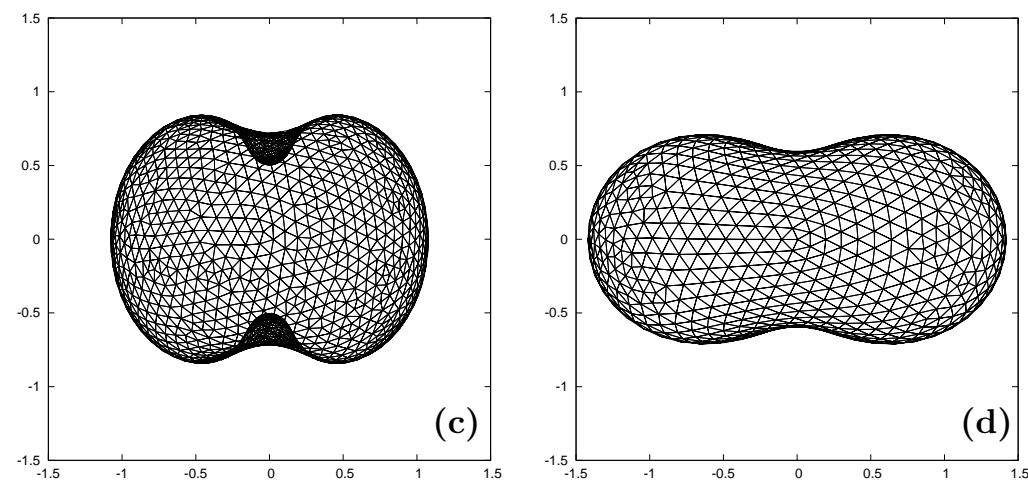

Fig. 4. Comparison of linear analysis (Solid) and nonlinear results (Dash-dotted) for the simulation in Fig. 3. (a) Scale factor $S(t)$; (b) Perturbation size $\bar{\delta}$; circles correspond to morphologies in Fig. 3 (a)-(d). (c) Nonlinear tumor morphology at $t=2.668$, with $S=4.78$ and $\bar{\delta}=0.496$; (d). Linear solution morphology (shown with a triangulated mesh) at the same time, $S=4.73$ and $\bar{\delta}=0.42$. Positive z-axis view. Reprinted with permission from Discrete and Continuous Dynamical Systems Series B, Li et al., Vol. 7, p. 600. Copyright 2007 American Institute of Mathematical Sciences. 

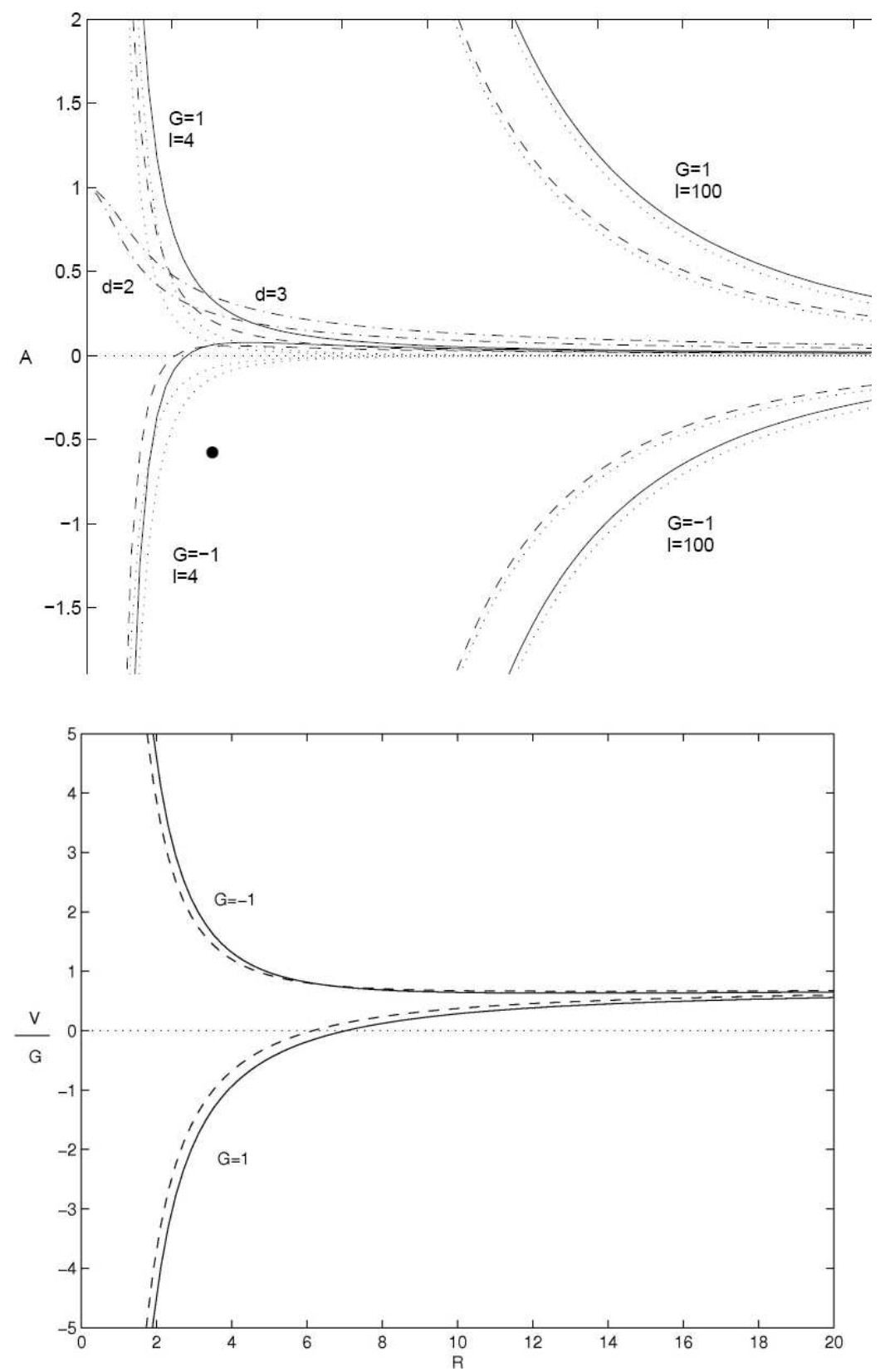

Fig. 5. Top: apoptosis parameter $A$ as a function of unperturbed radius $R$ from condition (16) for self-similar evolution; $d=2$ (dashed) and $d=3$ (solid); $G$ and $l$ labelled. Asymptotic behaviors are dotted (see [59]). The two solid curves labelled with values of $d$ correspond to stationary radii. Reprinted with permission from Discrete and Continuous Dynamical Systems - Series B, Li et al., Vol. 7, p. 598. Copyright 2007 American Institute of Mathematical Sciences. Bottom: corresponding growth velocity $G^{-1} V$ for $l=4$. 

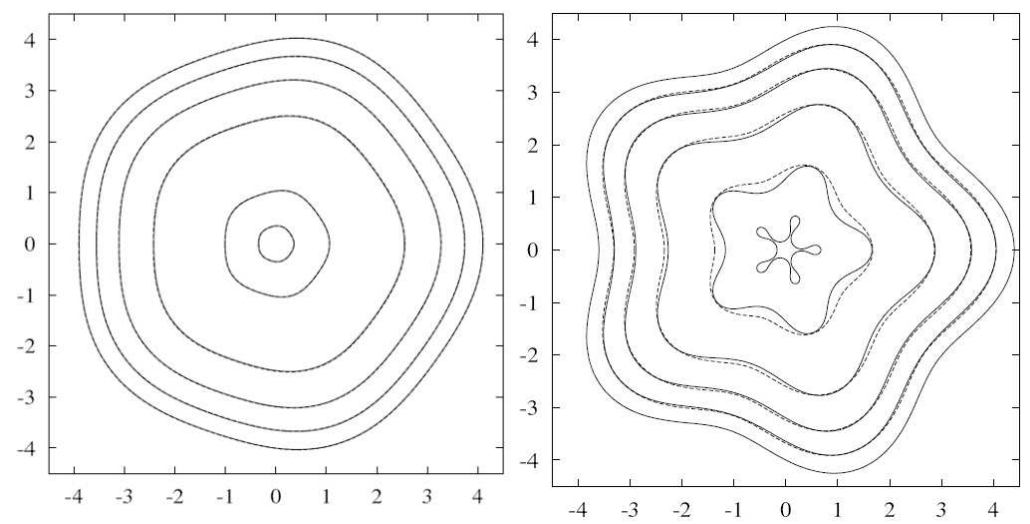

Fig. 6. Left: self-similar shrinkage for $R_{0}=4$ and $\delta_{0}=0.2$ ( $t=0$ to 0.96 shown). Right: Unstable shrinkage for $R_{0}=4$ and $\delta_{0}=0.4(t=0$ to 0.99$)$. The solid curves correspond to the nonlinear solution and the dashed curves to the linear. In both cases, $d=2, G=1, l=5$ and the evolution is in the low-vascularization regime. $A=A(l, G, R)$ given in (16) and plotted in Fig. 5 (top). Reprinted from Journal of Mathematical Biology, Cristini et al. Vol. 46, p. 215, Copyright 2003 Springer. With kind permission of Springer Science and Business Media. 


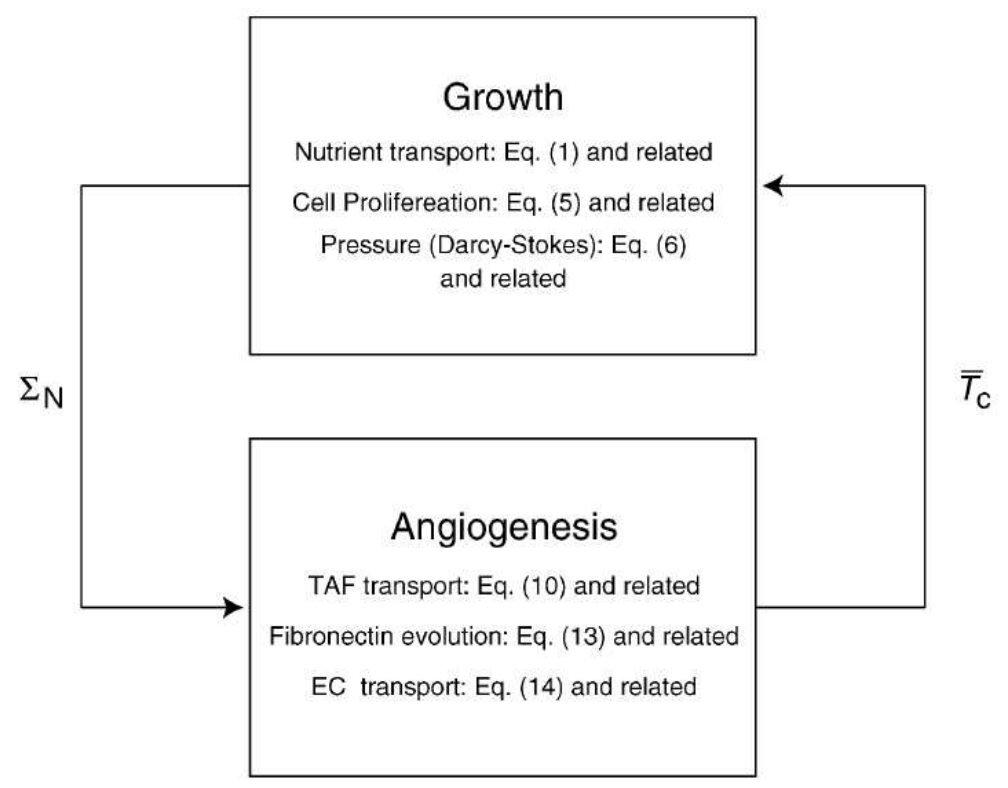

Fig. 7. Progression of a solid tumor is the result of coupling of growth and angiogenesis components that are mainly linked by the instantaneous position of the necrotic $\operatorname{rim} \Sigma_{N}$ and the blood-to-tissue nutrient transfer rate $\bar{T}_{c}$. Reprinted with permission from Bull. Math. Biol., Zheng et al. Vol. 67, p. 214, Copyright 2005 Springer (with kind permission of Springer Science and Business Media). 


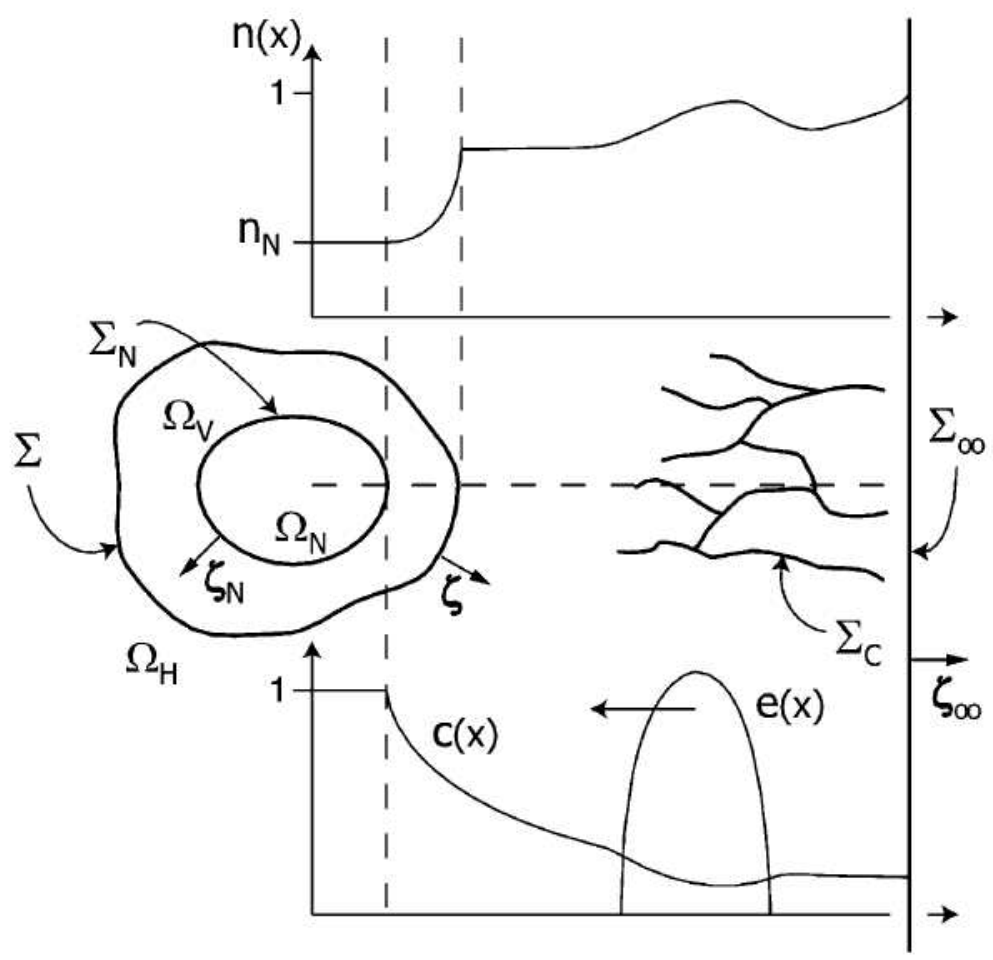

Fig. 8. Schematic (not to scale) of a necrotic tumor in transition from avascular to vascular growth gives a basic description of the model. Disjoint regions $\Omega_{H}, \Omega_{V}$ and $\Omega_{N}$ represent healthy tissue, viable tumoral tissue, and necrotic core domains, respectively. Tumor region is $\Omega_{T}=\Omega_{V} \cup \Omega_{N}$. $\Sigma_{\infty}$ is far field boundary, $\Sigma$ is tumor interface, and $\Sigma_{N}$ is necrotic rim. Capillaries are defined on $\Sigma_{C}$. For illustration, nutrient concentration $n(x)$, TAF concentration $c(x)$, and endothelial cell density $e(x)$ are plotted along horizontal dashed line. Reprinted with permission from Bull. Math. Biol., Zheng et al. Vol. 67, p. 215, Copyright 2005 Springer (with kind permission of Springer Science and Business Media). 


\begin{tabular}{llll}
\hline \multicolumn{1}{c}{ Growth } & \multicolumn{2}{c}{ Angiogenesis } \\
\hline & & $D_{c}=\bar{D}_{c} /\left(\bar{\beta}_{\mathrm{D}} \mathcal{L}^{2}\right)$ & $2.5 \times 10^{3}$ \\
$\nu_{\mathrm{P}}^{\text {ang }}=\bar{\nu}_{\mathrm{P}}^{\text {ang }} / \bar{\nu}_{\mathrm{U}}$ & $2.5 \times 10^{-2}$ & $\beta_{\mathrm{U}}=\bar{\beta}_{\mathrm{U}} / \bar{\beta}_{\mathrm{D}}$ & 1.0 \\
$\nu_{\mathrm{P}}^{\mathrm{pre}}=\bar{\nu}_{\mathrm{P}}^{\text {pre }} / \bar{\nu}_{\mathrm{U}}$ & 0 & $\eta_{\mathrm{P}}=\bar{\eta}_{\mathrm{P}} / \bar{\lambda}_{\mathrm{M}}$ & $2.5 \times 10^{-3}$ \\
$n_{\mathrm{C}}=\bar{n}_{\mathrm{C}} / \bar{n}_{\infty}$ & 1.0 & $\eta_{\mathrm{U}}=\bar{\eta}_{\mathrm{U}} / \bar{\lambda}_{\mathrm{M}}$ & $5 \times 10^{-3}$ \\
$n_{\mathrm{N}}=\bar{n}_{\mathrm{N}} / \bar{n}_{\infty}$ & $2.5 \times 10^{-2}$ & $\eta_{\mathrm{N}}=\bar{\eta}_{\mathrm{N}} / \bar{\lambda}_{\mathrm{M}}$ & $3 \times 10^{-3}$ \\
$p_{\mathrm{C}}^{\text {ang }}=\bar{p}_{\mathrm{C}}^{\text {ang }} / \bar{p}_{\mathrm{T}}$ & 1.0 & $D_{e}=\bar{D}_{e} /\left(\bar{\lambda}_{\mathrm{M}} \mathcal{L}^{2}\right)$ & $1.5 \times 10^{-3}$ \\
$p_{\mathrm{C}}^{\mathrm{pre}}=\bar{p}_{\mathrm{C}}^{\mathrm{pre}} / \bar{p}_{\mathrm{T}}$ & 0 & $\chi_{c}=\frac{\bar{\chi}_{c} \bar{c}_{0}}{\bar{\lambda}_{\mathrm{M}} \mathcal{L}^{2}}$ & 2.0 \\
$\lambda_{\mathrm{A}}=\bar{\lambda}_{\mathrm{A}} / \bar{\lambda}_{\mathrm{M}}$ & 0 & $\chi_{f}=\frac{\bar{\chi}_{f} \bar{f}_{0}}{\bar{\lambda}_{\mathrm{M}} \mathcal{L}^{2}}$ & 0.3 \\
$\lambda_{\mathrm{N}}=\bar{\lambda}_{\mathrm{N}} / \bar{\lambda}_{\mathrm{M}}$ & 0.25 & $\rho_{\mathrm{D}}=\bar{\rho}_{\mathrm{D}} / \bar{\lambda}_{\mathrm{M}}$ & $1.0 \times 10^{-3}$ \\
$\gamma=\bar{\gamma} /\left(\bar{\lambda}_{\mathrm{M}} \mathcal{L}^{3} / \bar{\mu}\right)$ & 0.25 & $\rho_{\mathrm{P}}=\bar{\rho}_{\mathrm{P}} / \bar{\lambda}_{\mathrm{M}}$ & $1.0 \times 10^{-2}$ \\
& & $c^{\star}=\bar{c}^{\star} / \bar{c}_{0}$ & 0.1 \\
& & $\rho_{\mathrm{N}}=\bar{\rho}_{\mathrm{N}} / \bar{\lambda}_{\mathrm{M}}$ & $1.0 \times 10^{-2}$ \\
\hline
\end{tabular}

Fig. 9. Complete model parameters used in the full simulation, the results for which are displayed in Fig. 10. Reprinted with permission from Bull. Math. Biol., Zheng et al. Vol. 67, p. 239, Copyright 2005 Springer (with kind permission of Springer Science and Business Media). 

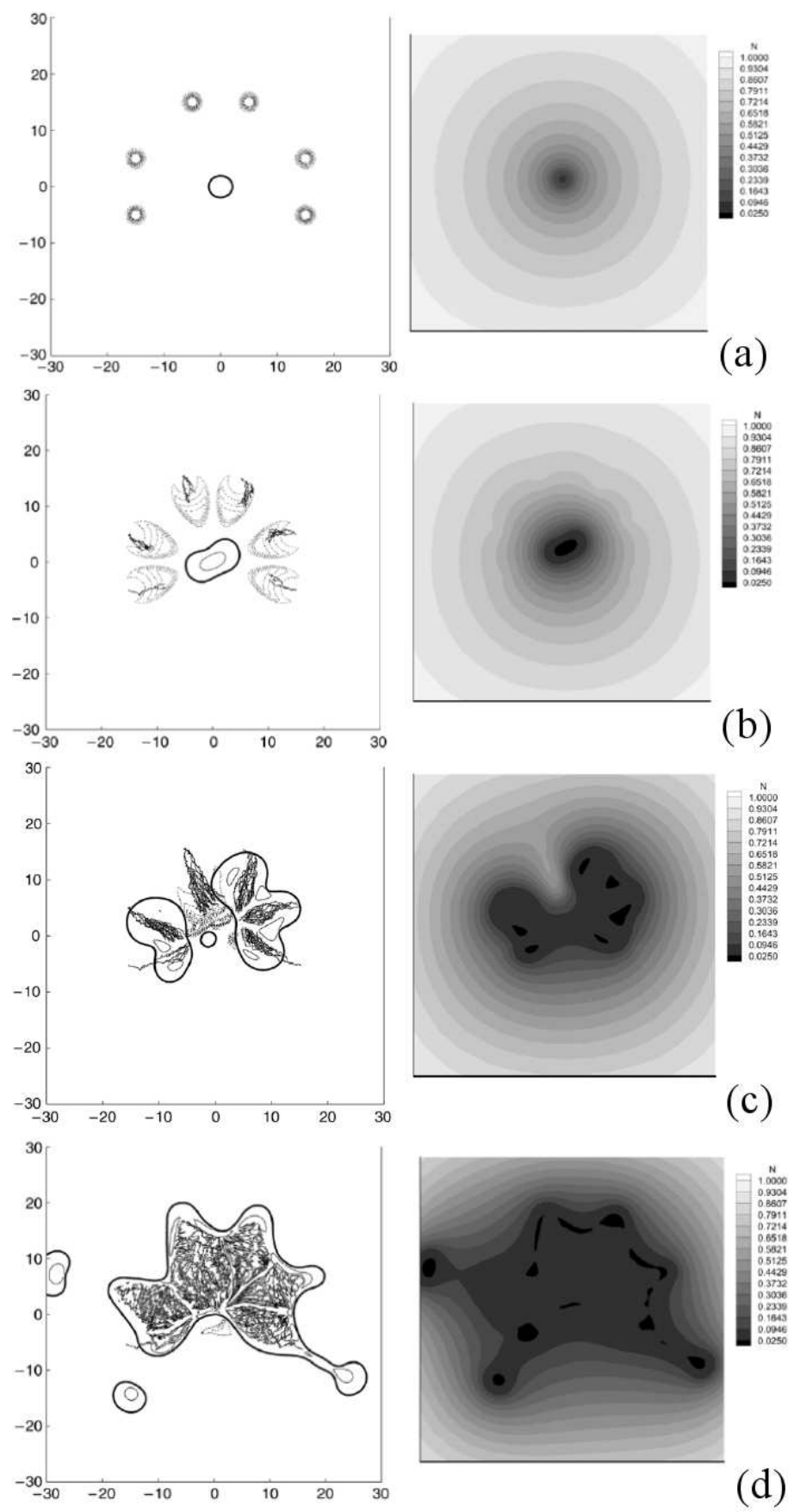

Fig. 10. Simulation of growth, neo-vascularization and infiltration of the brain by a malignant glioma. Parameters are listed in Fig. 9. (a) Time $t=0$; (b) Time $t=200$; (c) Time $t=400$ shows penetration into the tumor by the neovasculature and splitting of tumor into three fragments due to diffusional instability; (d) Time $t=900$ shows that tumor fragments have coopted the neo-vasculature, rejoined, and reached a nearly stationary state of centimeter-size. Small clusters separate from the main tumor and migrate up nutrient concentration gradients. Reprinted with permission from Bull. Math. Biol., Zheng et al. Vol. 67, pp. 240, 242, 244, 247, Copyright 2005 Springer (with kind permission of Springer Science and Business Media). 

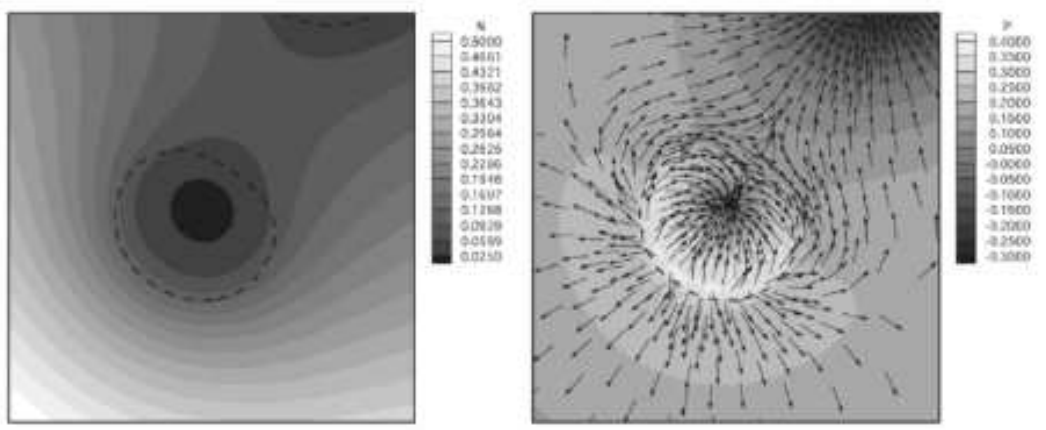

Fig. 11. Magnified view of the infiltrating tumor fragment in the bottom left of Fig. 10(d) $(t=900)$. Left: nutrient levels $(N)$ and tumor fragment interface (dashed). Right: cell velocity (arrows) and interstitial pressure $(P)$. A net migration of this fragment away from the main tumor body is the consequence of cell birth on the leading edge and death on the trailing edge due to the gradient of nutrient concentration. Note the high pressure in the proliferative leading edge, and the mass sink due to necrosis in the trailing edge. Reprinted with permission from Bull. Math. Biol., Zheng et al. Vol. 67, p. 254, Copyright 2005 Springer (with kind permission of Springer Science and Business Media). 


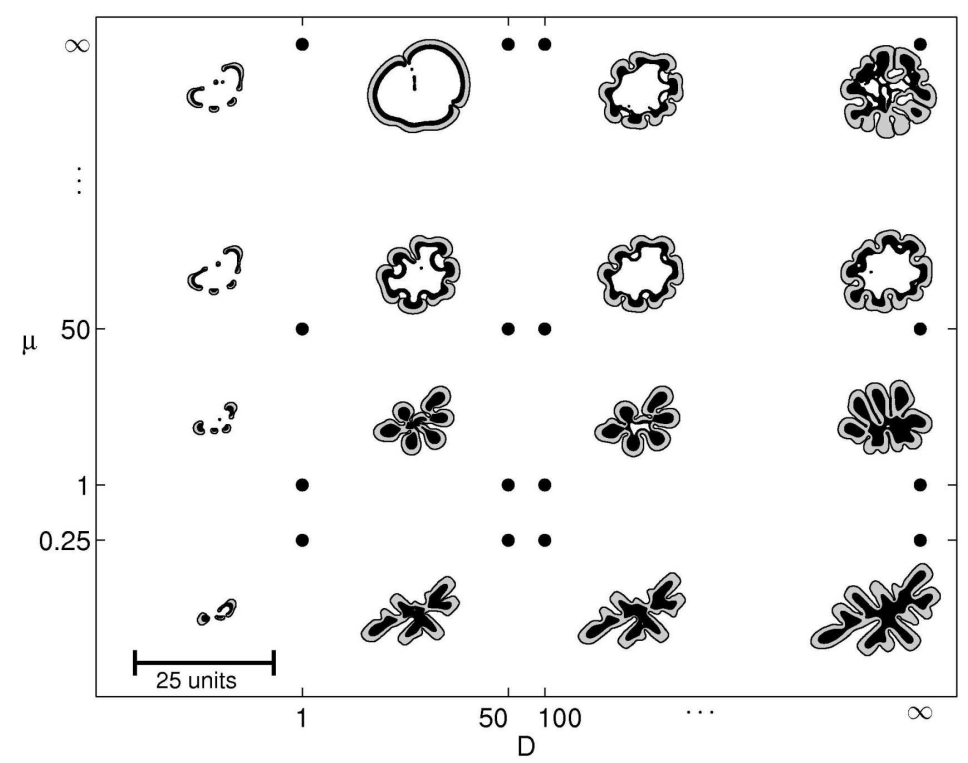

Fig. 12. Tumor morphological response to the microenvironment. External tissue nutrient diffusivity $D$ increases from left to right, and the external tissue mobility $\mu$ increases from bottom to top. Three major morphologies are observed: fragmenting growth (left), invasive fingering (lower right), and compact/hollow (upper right). All tumors are plotted to the same scale, where the indicated length is $25 L \approx 0: 5 \mathrm{~cm}$. Necrosis in black, viable region in gray. Reprinted with permission from Macklin \& Lowengrub, J. Theor. Biol. Vol. 245, p. 687, (2007). Copyright (C) Elsevier. 

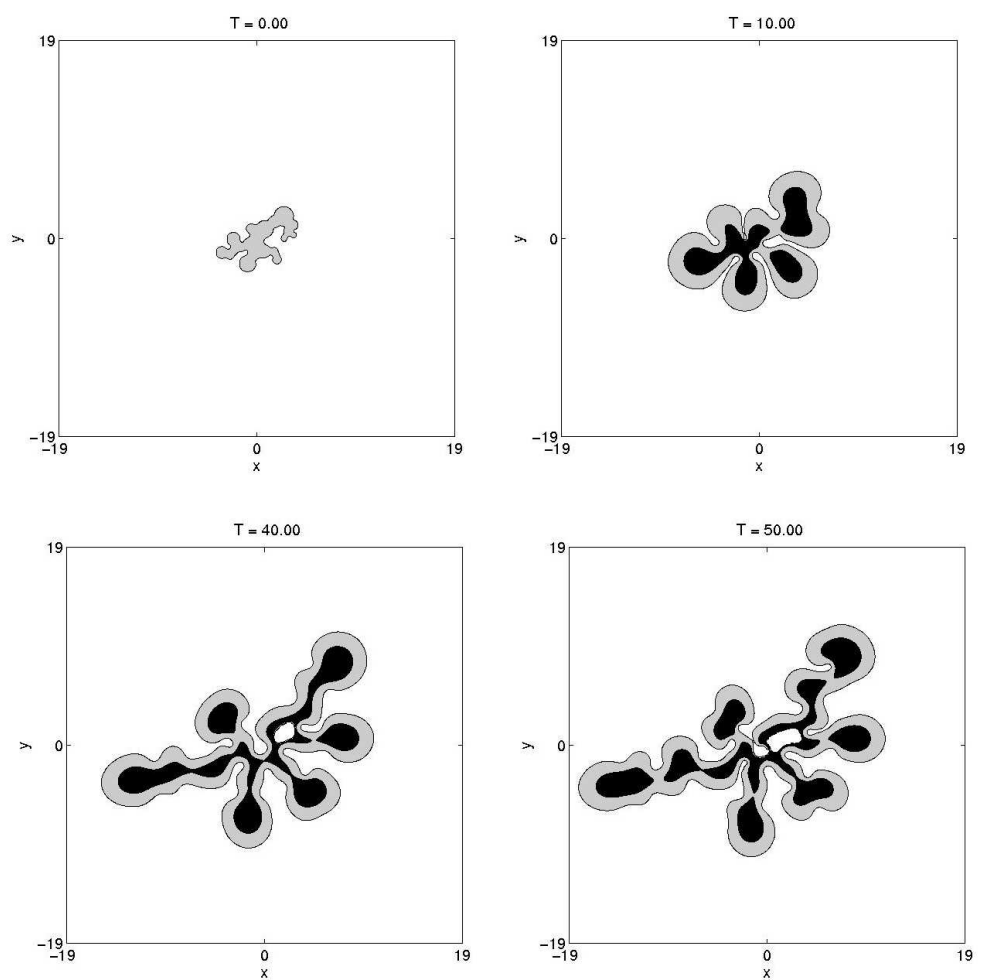

Fig. 13. Long-time simulation of invasive, fingering growth into nutrient-rich $(D=$ $50)$, low-mobility $(\mu=1)$ tissue. $G=20 ; G_{N}=1 ; N=0.35$, and $A=0$. Necrosis in black, viable region in gray. Reprinted with permission from Macklin \& Lowengrub, J. Theor. Biol. Vol. 245, p. 693, (2007). Copyright (c) Elsevier. 


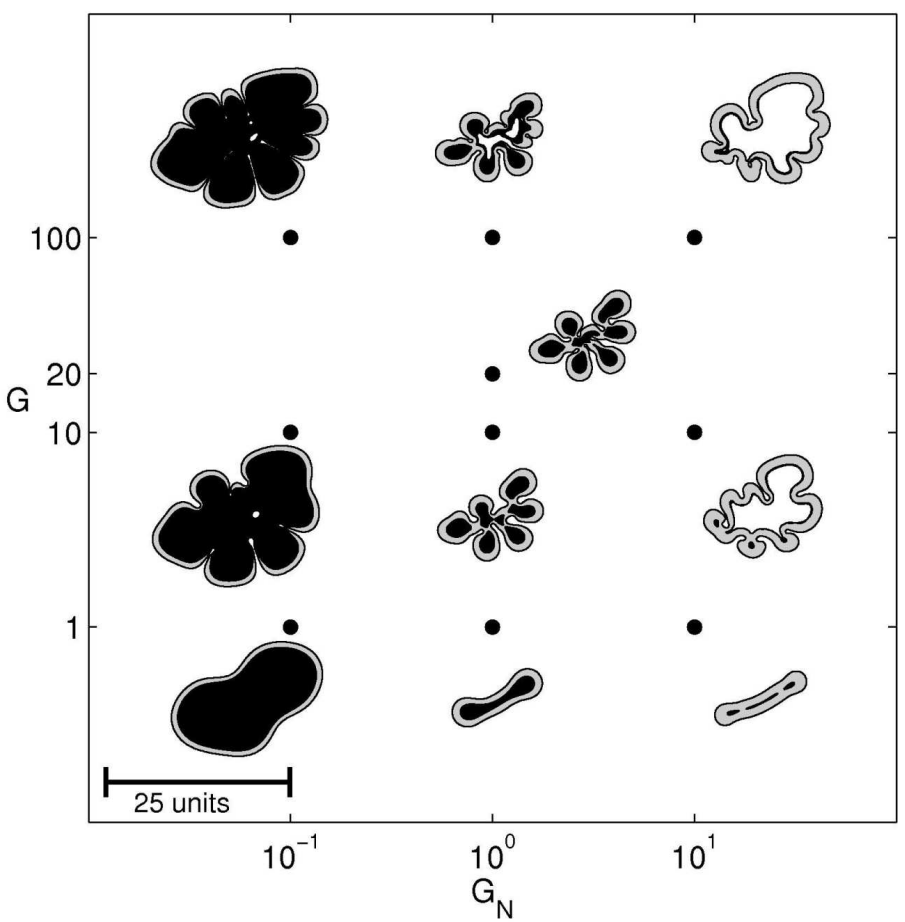

Fig. 14. Parameter study in $G$ and $G_{N}$ for invasive, fingering tumor growth into nutrient-rich, low-mobility tissue $(D=50 ; \mu=1)$. The tumor aggressiveness parameter $G$ increases from bottom to top, and the necrotic degradation parameter $G_{N}$ increases from left to right. Necrosis in black, viable region in gray. Reprinted with permission from Macklin \& Lowengrub, J. Theor. Biol. Vol. 245, p. 696, (2007). Copyright (c) Elsevier. 

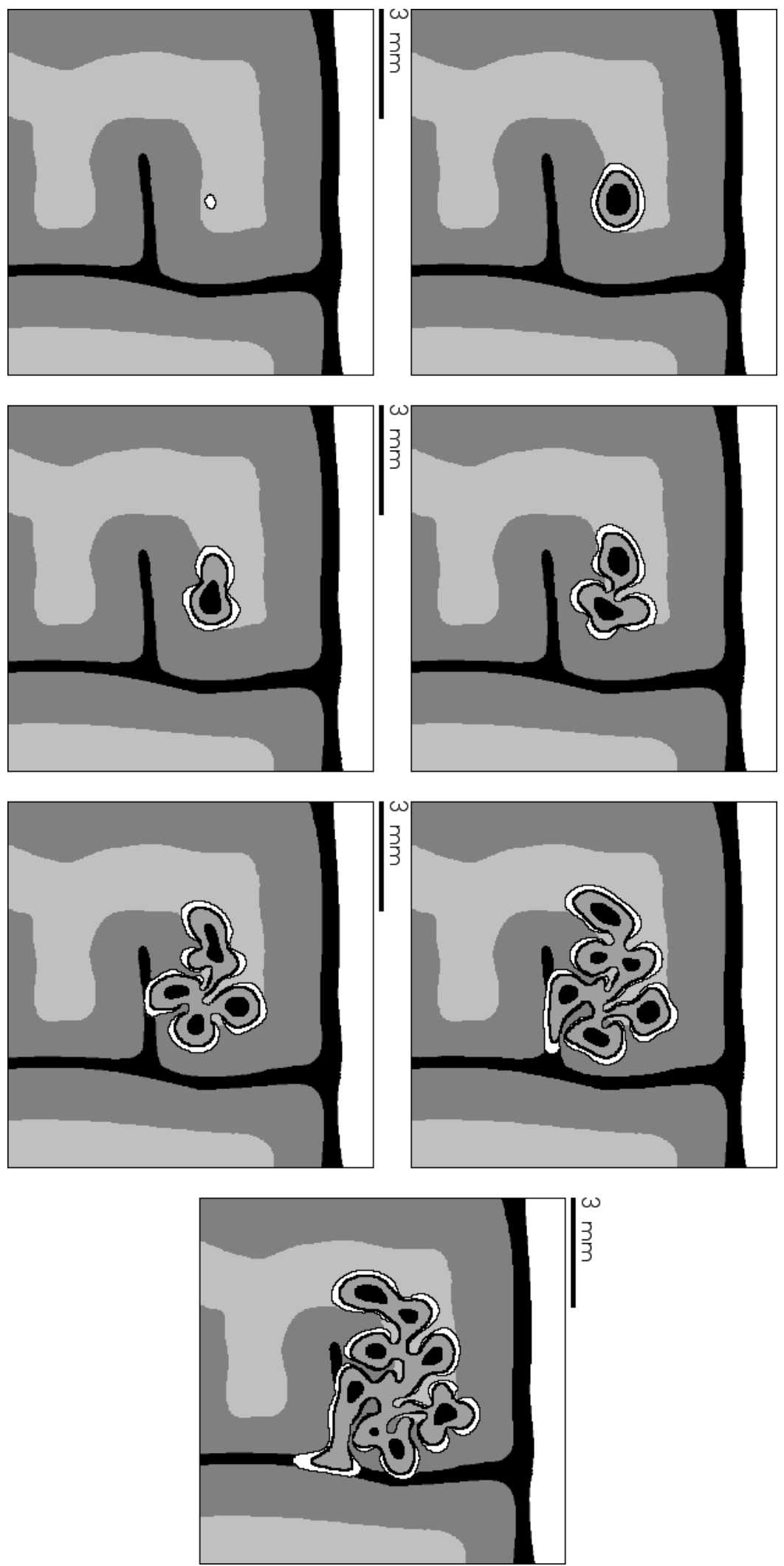

Fig. 15. Tumor simulation from $t=0.0$ days (top left) to $t=60.0$ days (bottom right) in 10 day increments. White band on the right of each frame models a rigid material such as the skull; black denotes an incompressible fluid (e.g., cerebrospinal fluid); light and dark gray regions represent tissues of differing biomechanical properties (e.g., white and gray matter). Tumor tissue is shown growing in the middle right with viable (outer layer, white), hypoxic (middle layer, gray), and necrotic (core, black) regions. Reprinted from Macklin \& Lowengrub (in review) [136]. 


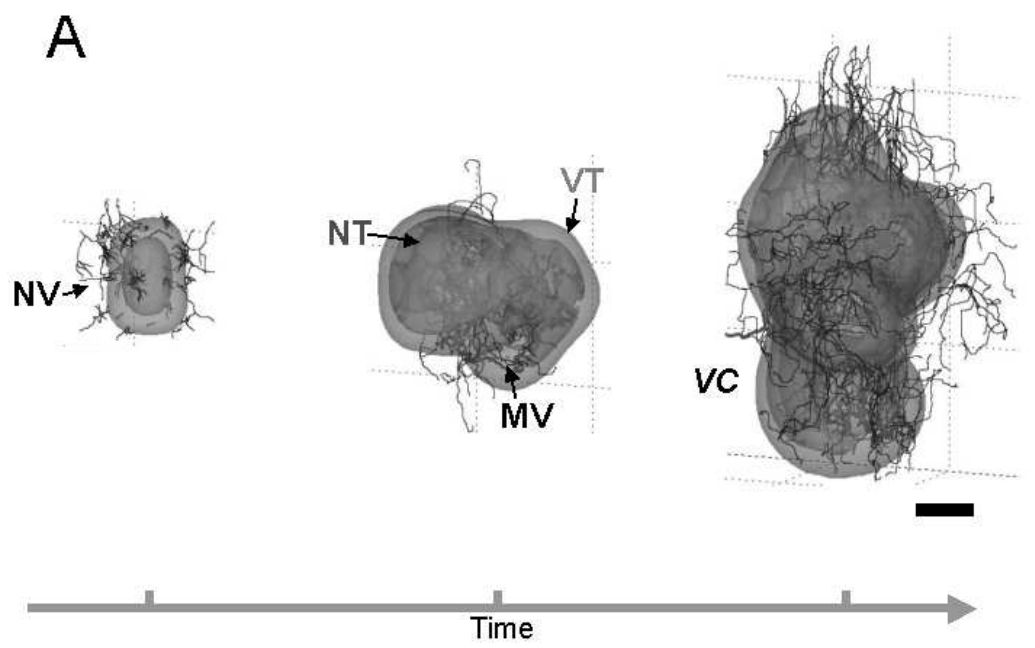

Fig. 16. Multiscale 3-D computer model predicts gross morphologic features of a growing glioblastoma. Viable (VT) and necrotic (NT) tissue regions, and vasculature (MV: mature blood-conducting vessels; NV: new non-conducting vessels) are shown. Time sequence (from left to right, over a period of 3 months) reveals that the morphology is affected by successive cycles of neovascularization, vasculature maturation, and vessel cooption $(V C)$. Bar, $250 \mu \mathrm{m}$. Reprinted from NeuroImage, Frieboes et al., in press, Copyright 2007, with permission from Elsevier. 



\section{Index}

adaptive mesh, 4, 14, 25

adhesion, 2, 3, 5, 27, 33, 40, 43

in glioma, 23

modeling of, 14, 17, 29, 30, 32, 41

adhesive force, 4, 8, 17, 21, 23, 37

adhesiveness, 23, 33

analysis

linear, 2, 7-9

weakly nonlinear, 2

anastomosis, 20, 24, 39, 42, 43

angiogenesis, 3, 4, 9, 11, 13, 14, 16, 18, $22,23,39,43$

angiogenic factors, 4, 14, 39, 42, 43

apoptosis, 3-5, 7, 8, 11, 12, 15, 20, 23, $29,30,39$

rate, $5,12,17,27,32,40,42$

avascular growth, $6,28,30,36$

boundary, 28

computational, 22

condition, 14, 17-19, 27, 30, 35, 41

far field, 16,30

necrotic, 29

tumor, 17, 29-32, 35, 42-44

boundary integral, 2, 4, 5, 13

Brinkman equation, 17

bulk energy, 41

cancer, 1

growth, 1

invasion, 1, 9, 13, 17, 22, 23, 34, 36, 37

progression, 1

treatment response, 1 capillary

branching, 20, 24

growth, 19, 22, 25

length, 23

position, 20

sprout, 14, 19, 20

tip, 19

cell

phenotype, 1, 45

chemotaxis, 11, 14, 17, 19, 22, 38, 39, 41-44

chemotherapy, $3,25,37,45$

conservation laws, 38

convection, 14, 39

curvature, 5, 8, 9, 17, 27, 30, 41, 42

Darcy's law, 22, 28, 30, 38, 41

Darcy-Stokes law, 17

differential equations

ordinary, 2

partial, 2, 27

diffusion, 4, 17-19, 27, 38, 39

angiogenic factors, 11, 18

constant, 16, 18, 19, 21, 31, 40

gradients, 1, 43, 44

nutrient, 8, 14, 32, 33

diffusional

growth, 4, 6, 8, 9, 12, 14, 24, 25

instability, 21, 22, 24, 26

length, 5, 21, 43

diffusivity, 18

nutrient, 28, 30, 31, 33

Dirichlet-Neumann map, 4 
ECD, 14, 20, 24

ECM, 14, 22, 27, 28, 31-34, 36-39, 41

EGFR, 23

endothelial

cell density, 14, 18, 20

cells, 11, 14, 15, 18, 19, 22, 24, 39, 42

equation

reaction-diffusion, 4, 14, 18-20, 27, $35,38,39,42$

extracellular matrix, 14, 17, 18, 22, 23

FAK, 23, 26

FCCMU, 37-40, 42

fibronectin, 15, 18, 20, 21, 40, 42

fingering, 4, 13, 14, 25, 26, 31-34, 36, 37,43

width, $33,34,36$

finite difference, 19, 42

finite element, 14, 20, 25

first principles, 44

flux, 5, 10, 11, 40, 41

functional relationships, 39, 45

genetic

expression, 2, 26-28, 31, 32, 45

instability, 2

ghost cell/level set, 3, 27

glioma, 3

glucose, 14, 21, 25, 28

growth factors, $25,26,39$

haptotaxis, 19, 21, 22, 38, 39, 41, 42, 44

HGF, 37

histopathology, 38, 42, 44

hydraulic conductivity, 17

hypoxia, $1,3,26,28,29,35,36,39,45$

interface

necrotic, 18

tumor-host, $2-5,7,11,16,17,20,38$, 41,43

invasion

determinants of, 45

mechanisms of, 26

rate of, 35,36

isotropic stress, 41

Laplace-Young condition, 17, 30

level set, 3, 5, 14, 20, 25, 27 lysis rate, 39

MAPK, 23

mechanotaxis, 41

microenvironment, 1-3, 20, 26-28, 30-33, 35-38, 43-45

microvasculature, 1

mitosis, 3-5, 14, 15, 17, 35, 39

rate, $3-5,17,21,26,31,40,42,43$

mobility, 3, 5, 17, 20, 23, 26, 28, 29, $31-33,36,41$

model

2-D, 3, 4, 8, 27

3-D, 2-4, 9, 37, 38, 43, 44

agent-based, 2

angiogenesis, 3, 15, 20, 22, 25, 38, 42

cellular automata, 2

continuum, 2-4, 14, 19, 41

diffuse interface, 3,41

hybrid continuum-discrete, $3,14,19$, 38,42

input parameters, 44

lattice-free, 38,42

mathematical, 1, 2, 5, 17, 45

mixture, 2, 5, 28

multiphase, 2

multiscale, 38, 39, 43-45

nonlinear, 2

nonlinear-elastic/plastic, 41

single-phase, 2

Stokes, 41

tumor growth, 2-4, 8, 15, 25, 27, 30, $35,39,43$

vasculature, 42

viscoelastic, 41

morphology, 1, 3, 9, 10, 22, 25-28,

$30-32,36,37,44,45$

compact, 34, 43

compact/hollow, 34, 36

diagram, 30, 31

glioma, 44

in prognosis, 45

instability, 2, 3, 8, 9, 12, 13, 21, 27, $32-36,43$

prediction of, 8,44

MRI, 39, 44

necrosis, 5-7, 14-19, 21-23, 25, 27-31, 34-36, 39, 40, 43, 44 
degradation, 34

neo-vasculature, 22-24, 39, 43

non-necrotic, $3,4,13,32$

nonlinearity, 10-12

nutrient, 1, 4, 11, 14-17, 20-26, 28, 29, $31,32,35,36,38,39,42,43$

consumption, 16, 21

transfer, 15, 16, 20-25, 28, 40

transport, 15

oxygen, 1, 14, 28, 37, 39, 40

peri-necrotic, 39, 42, 43

perturbation, 7

asymmetric, 2

decay, $9,10,13$

growth, 8-12

linear, 10

nonlinear, 10, 12

size, $7,9,10$

stationary, 10, 11

pinch-off, 8, 10-12

pressure, 5, 7, 16, 17, 20, 21, 23, 24, 26, $28-33,35,36,38,41,43$

blood, 16,24

interstitial, 15, 21

proliferation, 1, 2, 15-17, 21, 23, 26, 28-30, 32, 34-37, 39, 43-45

endothelial, 24, 39, 42

random walk, 19, 42

relaxation

mechanisms, 3,5

rate, 5

self-similar evolution, 4, 11-13

simulation

$2-\mathrm{D}, 3$

$3-\mathrm{D}, 9,44$

automata, 3

boundary integral, $3,8,13,14$

computer, 1, 2, 20, 24-26, 34, 35, 43

nonlinear, 2, 3, 8-10, 12, 13

parameters, 42

SOAM, 42

stationary radius, $7,10,12$

shape, 11

solution, 7

state, $6,10-12$

surface

area, 9,33

force, 14

integrals, 4

tension, 4, 8, 17, 21, 23, 30, 41

tumor, 4, 5, 8, 9, 34, 38

TAF, $14,15,18,20,22$

therapy, 12

adhesion, 32, 33, 37

anti-angiogenic, 1, 32, 36, 37

anti-invasive, 37

chemo-, $3,25,37$

radiation, 45

time scale, 5, 15, 21, 35, 43

tortuosity, 42, 43

tumor

fragmentation, 12, 22, 25, 26, 31, 32, 34,36

growth, 3, 4, 6, 9, 11-14, 20-22, 24, $30,32,33,35,37$

invasion, 1, 9, 13, 17, 22, 23, 34, 36, 37

vascularization, $4,5,12,25$

high, 4, 6, 12

low, 6, 8-10, 12

moderate, 6

vasculature, 16, 18, 20, 24, 42, 43

architecture, 42

modeling of, 42

normalization, 36

transfer from, 25

VEGF, 14, 39, 42

velocity, $6,12,17,29,39$

cell, 15, 16, 19, 20, 38, 40

convective, 19

field, 28, 29, 32

growth, $6,11,12$

normal, 4, 5, 27, 29, 30

water, 40 